Supporting information for

\title{
[4]Cyclo-N-alkyl-2,7-carbazole: Influence of the alkyl chain length on the structural, electronic and charge transport properties
}

Fabien Lucas, ${ }^{\mathrm{a}}$ Nemo McIntosh, ${ }^{\mathrm{b}}$ Emmanuel Jacques, ${ }^{\mathrm{c}}$ Christophe Lebreton, ${ }^{\mathrm{c}}$ Benoît

Heinrich, ${ }^{\mathrm{d}}$ Bertrand Donnio, ${ }^{\mathrm{d}}$ Olivier Jeannin, ${ }^{\mathrm{a}}$ Joëlle Rault-Berthelot, ${ }^{\mathrm{a}}$ Cassandre Quinton, ${ }^{\mathrm{a}}$ Jérôme Cornil, ${ }^{\mathrm{b}}$ Cyril Poriel ${ }^{\mathrm{a}^{*}}$

a Univ Rennes, CNRS, ISCR-UMR CNRS 6226, F-35000 Rennes, France

email: cyril.poriel@univ-rennes1.fr

${ }^{\mathrm{b}}$ Laboratory for Chemistry of Novel Materials, Materials Research Institute, University of Mons, Mons, Belgium

c Univ Rennes, CNRS, IETR-UMR 6164, F-35000 Rennes, France

d Institut de Physique et Chimie des Matériaux de Strasbourg (IPCMS), UMR 7504, CNRS-Université de Strasbourg, 23 rue du Loess, BP 43, 67034 Strasbourg Cedex 2, France 


\section{Table Of Contents}

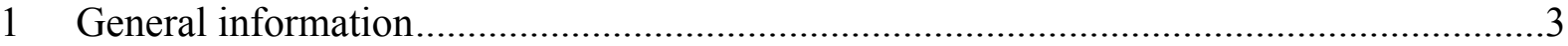

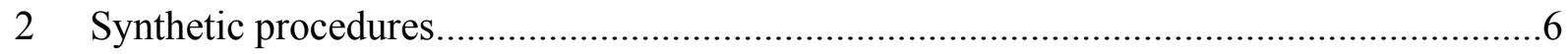

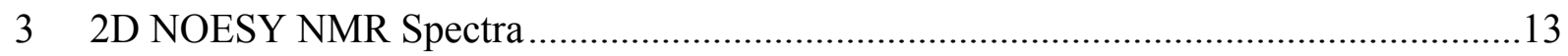

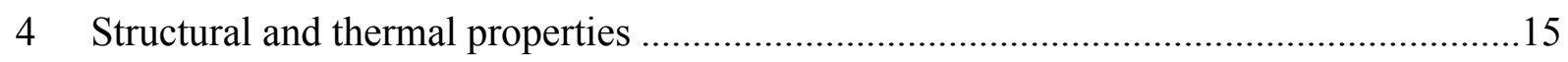

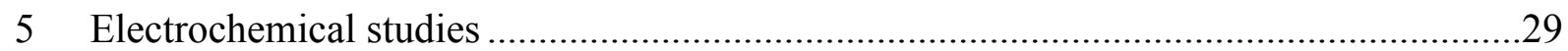

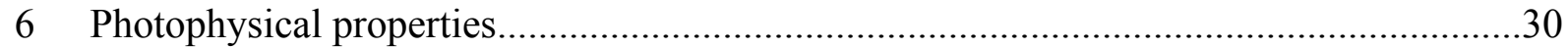

7 Organic field effect transistor and space-charged-limited current diode measurements...32

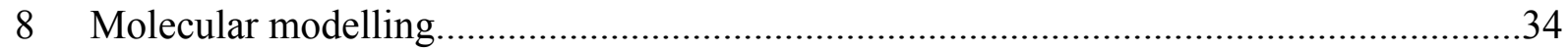

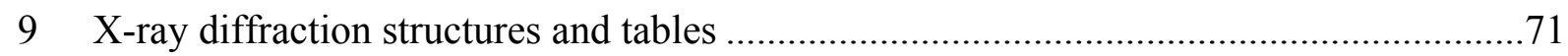




\section{General information}

\section{Synthesis}

All manipulations of oxygen and moisture-sensitive materials were conducted with a standard Schlenk technique. All glassware was kept in an oven at $80^{\circ} \mathrm{C}$. Argon atmosphere was generated by three repetitive cycles of vacuum/Argon using a schlenck ramp. Commercially available reagents and solvents were used without further purification other than those detailed below. THF and toluene were obtained through a PURE SOLV ${ }^{\mathrm{TM}}$ solvent purification system. Light petroleum refers to the fraction with $\mathrm{bp} 40-60^{\circ} \mathrm{C}$. Analytical thin layer chromatography was carried out using aluminum backed plates coated with Merck Kieselgel 60 GF254 and visualized under UV light (at 254 and $360 \mathrm{~nm}$ ). Flash chromatography was carried out using Teledyne Isco CombiFlash ${ }^{\circledR}$ Rf 400 (UV detection 200-360nm), over standard silica cartridges (Redisep ${ }^{\circledR}$ Isco or Puriflash ${ }^{\circledR}$ columns Interchim). In order to be purified through a recycling size exclusion chromatography apparatus, the compounds were solubilized in HPLC grade chloroform (stabilized with ethanol). Prior to injection, the solution was filtered through a 0.45 $\mu \mathrm{m}$ PTFE filter. Purification was performed on a LC-9160 II NEXT system from the Japan Analytical Industry Co., Ltd. (JAI) equipped with coupled UV-vis 4Ch NEXT through a set of two JAIGEL-2H columns at an elution rate of $10 \mathrm{~mL} \cdot \mathrm{min}^{-1}\left(\mathrm{CHCl}_{3}\right) .{ }^{1} \mathrm{H}$ and ${ }^{13} \mathrm{C} \mathrm{NMR}$ spectra were recorded using Bruker $300 \mathrm{MHz}$ instruments $\left({ }^{1} \mathrm{H}\right.$ frequency, corresponding ${ }^{13} \mathrm{C}$ frequency: $75 \mathrm{MHz}$ ); chemical shifts were recorded in ppm and $\mathrm{J}$ values in $\mathrm{Hz}$. The residual signals for the NMR solvents used are $5.32 \mathrm{ppm}$ (proton) and $54.00 \mathrm{ppm}$ (carbon) for $\mathrm{CD}_{2} \mathrm{Cl}_{2}, 7.26 \mathrm{ppm}$ (proton) and $77.16 \mathrm{ppm}$ (carbon) for $\mathrm{CDCl}_{3}$ and $77.16 \mathrm{ppm}$ (carbon), $2.090 \mathrm{ppm}$ (proton) for toluene-d8. In the ${ }^{13} \mathrm{C}$ NMR spectra, signals corresponding to $\mathrm{C}, \mathrm{CH}, \mathrm{CH}_{2}$ or $\mathrm{CH}_{3}$ groups, assigned from DEPT experiment, are noted. The following abbreviations have been used for the NMR assignment: $\mathrm{s}$ for singlet, $\mathrm{d}$ for doublet, $\mathrm{t}$ for triplet, $\mathrm{q}$ for quadruplet and $\mathrm{m}$ for multiplet. High resolution mass spectra were recorded at the Centre Régional de Mesures Physiques de l'Ouest (CRMPO-Rennes) on a Thermo Ficher Q-Exactive instrument or a Bruker MaXis $4 \mathrm{G}$ or a Bruker Ultraflex III.

\section{$X$-ray}

Crystals were picked up with a cryoloop and then frozen at $150 \mathrm{~K}$ under a stream of dry $\mathrm{N}_{2}$. Data were collected on a D8 VENTURE Bruker AXS diffractometer with Mo-K $\alpha$ radiation $(\lambda$ $=0.71073 \AA$ ).

Structures were solved by direct methods (SIR92) ${ }^{1}$ and refined (SHELXL-2014/7) ${ }^{2}$ by fullmatrix least-squares methods as implemented in the WinGX software package. ${ }^{3}$ An empirical absorption (multi-scan) correction was applied. Hydrogen atoms were introduced at calculated positions (riding model) included in structure factor calculation but not refined. Refinement parameters are summarized in Table S 23, Table S 24 and Table S 25.

Crystallographic data have been deposited with the Cambridge Crystallographic Data Centre as supplementary publication data: [4]C-Et-Cbz (CCDC 1893079), [4]C-Bu-Cbz (CCDC 2055214) and [4]C-Hex-Cbz (CCDC 2055215). Copies of the data can be obtained free of charge on application to CCDC, 12 Union Road, Cambridge CB2 1EZ, UK [fax: (+44) 1223336-033; e-mail: deposit@ccdc.cam.ac.uk].

Figures were generated with Mercury software 3.9.

\section{Spectroscopic studies}

Cyclohexane (spectroscopic grade, Acros), THF (spectroscopic grade, Acros), dichloromethane (spectroscopic grade, Acros), acetronitrile (spectroscopic grade, Acros), methanol (spectroscopic grade, Acros), 2-MeTHF (spectroscopic grade, sigma aldrich), $1 \mathrm{~N}$ solution of 
sulfuric acid in water (Standard solution, Alfa Aesar), and quinine sulfate dihydrate (99+\%, ACROS organics) were used without further purification.

UV-visible spectra were recorded using an UV-Visible spectrophotometer SHIMADZU UV-1605. Molar extinction coefficients $(\varepsilon)$ were calculated from the gradients extracted from the plots of absorbance $v s$ concentration with five solutions of different concentrations for each sample and at least two mother solutions were prepared.

$$
A=\varepsilon \times l \times C
$$

Above, 1 refers to the path length and $\mathrm{C}$ to the sample concentration.

Emission spectra were recorded with a HORIBA Scientific Fluoromax-4 equipped with a Xenon lamp.

Quantum yields in solution (øsol) were calculated relative to quinine sulfate (øref $=0.546$ in $\mathrm{H}_{2} \mathrm{SO}_{4} 1 \mathrm{~N}$ ). øsol was determined according to the following equation,

$$
\phi s o l=\phi r e f \times \frac{\operatorname{Grad}_{s}}{\operatorname{Grad}_{r}} \times\left(\frac{\eta_{s}}{\eta_{r}}\right)^{2}
$$

where subscripts $s$ and $r$ refer respectively to the sample and reference, Grad is the gradient from the plot of integrated fluorescence intensity $v s$ absorbance, $\eta$ is the refracting index of the solvent $\left(\eta_{s}=1.426\right.$ for cyclohexane). Five solutions of different concentration $(\mathrm{A}<0.1)$ of the sample and five solutions of the reference (quinine sulfate) were prepared. The integrated area of the fluorescence peak was plotted against the absorbance at the excitation wavelength for both the sample and reference. The gradients of these plots were then injected in the equation to calculate the reported quantum yield value for the sample.

Absolute quantum yields were recorded using a deported HORIBA Scientific Quanta-Phi integrating sphere linked to the Fluoromax-4.

Fluorescent decay measurements were carried out on the HORIBA Scientific Fluoromax-4 equipped with its TCSPC pulsed source interface.

Spin-coated film were prepare form a $1 \mathrm{~g} / \mathrm{mL}$ solution using a Labspins Tournette from Süss Microtec. Evaporated thin films of nanoring were prepared using the procedure described below for the fabrication of OFET devices.

IR spectra were recorded on a Bruker Vertex 70 using a diamond crystal MIRacle ATR (Pike).

\section{Electrochemical studies}

Electrochemical experiments were performed under argon atmosphere using a Pt disk electrode (diameter $1 \mathrm{~mm}$ ). The counter electrode was a vitreous carbon rod. The reference electrode was either a silver wire in a $0.1 \mathrm{M} \mathrm{AgNO}_{3}$ solution in $\mathrm{CH}_{3} \mathrm{CN}$ for the studies in oxidation or a Silver wire coated by a thin film of $\mathrm{AgI}$ (silver(I)iodide) in a $0.1 \mathrm{M} \mathrm{Bu}_{4} \mathrm{NI}$ solution in DMF for the studies in reduction. Ferrocene was added to the electrolyte solution at the end of a series of experiments. The ferrocene/ferrocenium $\left(\mathrm{Fc} / \mathrm{Fc}^{+}\right)$couple served as internal standard. The three electrodes cell was connected either to a PAR Model 273 potentiostat/galvanostat (PAR, EG\&G, USA) monitored with the ECHEM Software or to a potentiostat/galvanostat (Autolab/PGSTAT101) monitored with the Nova 2.1 Software. Activated $\mathrm{Al}_{2} \mathrm{O}_{3}$ was added in the electrolytic solution to remove excess moisture. For a further comparison of the electrochemical and optical properties, all potentials are referred to the SCE electrode that was calibrated at $-0.405 \mathrm{~V}$ vs. $\mathrm{Fc} / \mathrm{Fc}^{+}$system. Following the work of Jenekhe, ${ }^{4}$ we estimated the electron affinity (EA) or lowest unoccupied molecular orbital (LUMO) and the ionization potential (IP) or highest occupied molecular orbital (HOMO) from the redox data. The LUMO 
level was calculated from: $\operatorname{LUMO}(\mathrm{eV})=-\left[\mathrm{E}_{\text {onset }}{ }^{\text {red }}(v s \mathrm{SCE})+4.4\right]$. Similarly the HOMO level was calculated from: $\mathrm{HOMO}(\mathrm{eV})=-\left[\mathrm{E}_{\text {onset }}{ }^{\mathrm{ox}}(v s \mathrm{SCE})+4.4\right]$, based on a SCE energy level of $4.4 \mathrm{eV}$ relative to the vacuum. The electrochemical gap was calculated from: $\Delta \mathrm{E}^{\mathrm{el}}=\mid \mathrm{HOMO}-$ LUMO (in eV).

\section{Theoretical modelling}

\section{a) Absorption spectrum simulation}

Full geometry optimization of the ground state and vibrational frequency calculation were performed with Density Functional Theory (DFT) ${ }^{5-6}$ using the hybrid Becke-3 parameter exchange functional ${ }^{7-9}$ and the Lee-Yang-Parr non-local correlation functional ${ }^{10}$ (B3LYP) implemented in the Gaussian 16 program suite, ${ }^{11}$ using the $6-31 \mathrm{G}(\mathrm{d})$ basis set and the default convergence criterion implemented in the program. To overcome the issue of the many possible conformation of the alkyl chains in the cyclocarbazoles, geometry optimization was performed starting from the X-ray diffraction crystal structure geometry. All stationary points were characterized as minima by analytical frequency calculations.

The energy strain was performed as described in the literature using the B3LYP functionals and the $6-311+\mathrm{G}(\mathrm{d}, \mathrm{p})$ basis set. ${ }^{12}$

Optical transition diagrams were obtained through TD-DFT calculations performed using the B3LYP functionals and the $6-311+\mathrm{G}(\mathrm{d}, \mathrm{p})$ basis set from the geometry of $\mathrm{S}_{0}$.

Calculations were carried out on the OCCIGEN calculator of the Centre Informatique National de l'Enseignement Supérieur (CINES (Montpellier) under project N²020-A0080805032).

Figures were generated with GaussView 6.0 and GaussSum 3.0.

\section{b) Transfer integral calculation}

Experimentally resolved crystal structures were used to estimate electronic couplings in the equilibrium structure but also to build the $3 \mathrm{X} 3 \mathrm{X} 3$ supercells on which we performed MD calculations. From the latter, we are able to compute the distribution of the electronic couplings induced by thermal motion at ambient temperature in each structure. This reveals by how much their value is spread by the activation of intermolecular vibrational modes. All the electronic couplings were computed at the Density Functional Theory level within a fragment approach ${ }^{13}$ using the B3LYP function and a DZ basis set, as implemented in the ADF (Amsterdam Density Functional) package. The first step of our analysis consisted in determining the most probable pathways for holes to move along and the dimensionality of the charge transport. To achieve this, we extended the experimental cell into a $3 \times 3 \times 3$ supercell and computed the electronic couplings for all close neighbors of all molecules localized in the pristine unit cell; in practice, dimers are considered when the closest atoms are within a 0-5 ^ range.

Molecular Dynamics simulations were performed with the Dreiding force field, as implemented in the Material Studio package. For all four investigated systems, we started by computing the charge distribution in the molecule using the Qeq method ${ }^{14-15}$. All default values were chosen in the force field except for the hydrogen van der Waals radius (changed from 3.195 to $2.83 \AA$ ) and the treatment of electrostatic interactions from the shielded to pure coulombic $(1 / \mathrm{r})$ regime. The output of this relaxation was used as input for a dynamic run in the NVT ensemble with the Nose thermostat. Once all MD runs were validated by ensuring that the lattice fluctuations occur around the equilibrium geometry, we extracted around 150 snapshots through the whole simulation, and computed couplings for inequivalent molecules in the crystal structure.

An important fact is to take into consideration when looking at the following distributions. What matters, in the scope of the hopping model is the absolute value of the electronic coupling. This is why some mean values of the Gaussian fits do not match the actual distributions. They were computed on the basis of data sets compounded solely by absolute values. We preferred to keep 
the histograms and fits appearance close to the raw data in order to emphasize the Gaussian character of these distributions

\section{Thermal analysis}

Thermal Gravimetric Analysis (TGA) was carried out by using a Q50 apparatus from TA instruments. TGA curved were measured at $5^{\circ} \mathrm{C} / \mathrm{min}$ under air.

Differential Scanning Calorimetry (DSC) was carried out by using a DSC Q1000 from TA instrument. DSC traces were measured at $5^{\circ} \mathrm{C} / \mathrm{min}, 3$ heating/cooling cycles were successively carried out under air.

The S/WAXS patterns were obtained with a transmission Guinier-like geometry. A linear focalized monochromatic $\mathrm{Cu} \mathrm{K \alpha l}$ beam $(\lambda=1.5405 \AA)$ was obtained using a sealed-tube generator $(600 \mathrm{~W})$ equipped with a bent quartz monochromator. The samples were filled in home-made sealed cells of $1 \mathrm{~mm}$ path. The sample temperature was controlled within \pm 0.01 ${ }^{\circ} \mathrm{C}$, and exposure times were varied from 4 to $24 \mathrm{~h}$. The patterns were recorded with a curved Inel CPS 120 counter gas-filled detector (periodicities up to $90 \AA$ ) and on image plates scanned by Amersham Typhoon IP with $25 \mu \mathrm{m}$ resolution (periodicities up to $120 \AA$ ). I(2q) profiles were obtained from images, by using home-developed software.

\section{Organic field effect transistors fabrication}

The fabrication process is described as follows: $150 \mathrm{~nm}$ thick aluminium layer was evaporated and patterned by conventional photolithography on a $5 \times 5 \mathrm{~cm}^{2}$ rigid glass substrate. Then, SU82000.5 Photoresist from Microchem ${ }^{16}$ was then spin-coated in order to obtain a $400 \mathrm{~nm}$ thick layer. $50 \mathrm{~nm}$ thick gold layer was then thermally evaporated and patterned by photolithography. Finally, the OSCs were deposited by evaporation under vacuum as a $40 \mathrm{~nm}$ thick layer.

\section{Synthetic procedures}

\section{General procedure for alkylation of 2,7-dibromo-9H-carbazole}

2,7-Dibromo-9H-carbazole (1.0 eq), $\mathrm{KOH}$ (3.0 eq) and tetrabutylammonium hydrogen sulfate $(0.1 \mathrm{eq})$ were charged in a round bottom flask. Then the corresponding brominated alkyl (2.5 eq) and dry DMF $(0.30 \mathrm{M})$ were added. The resulting mixture was stirred at $80^{\circ} \mathrm{C}$ overnight. After cooling to RT, a saturated solution of ammonium chloride was added. The organic layer was extracted with $\mathrm{CH}_{2} \mathrm{Cl}_{2}$. The organic layer was then washed with water, brine, dried over $\mathrm{MgSO} 4$, filtered and concentrated under reduced pressure. The crude was purified with flash chromatography on silica gel.

\section{2,7-dibromo-N-ethyl-carbazole}

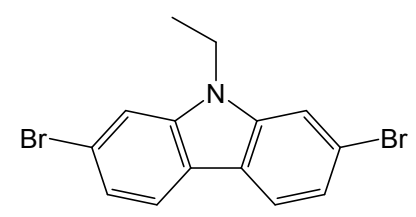

The title compound was synthesized using the general procedure for alkylation of 2,7-dibromo$9 \mathrm{H}$-carbazole.

2,7-Dibromo-9H-carbazole (2.00 g, $6.15 \mathrm{mmol}, 1.0 \mathrm{eq})$ KOH (1.04 g, $18.50 \mathrm{mmol}, 3.0 \mathrm{eq})$, tetrabutylammonium hydrogen sulfate $(209 \mathrm{mg}, 0.62 \mathrm{mmol}, 0.1 \mathrm{eq}), 1$-bromoethane $(1.14 \mathrm{~mL}$, $15.40 \mathrm{mmol}, 2.5 \mathrm{eq})$ and dry DMF $(20 \mathrm{~mL})$.

After purification flash chromatography on silica gel [column condition: silica cartridge ( $40 \mathrm{~g})$; solid deposit on Celite ${ }^{\circledR}$; $\lambda$ detection: $(254 \mathrm{~nm}, 280 \mathrm{~nm}) ; \mathrm{CH}_{2} \mathrm{Cl}_{2} /$ light petroleum (5:95) at 40 $\mathrm{mL} / \mathrm{min}]$, the title compound was obtained as a white solid $(2.10 \mathrm{~g}, 5.95 \mathrm{mmol})$; yield $97 \%$; ${ }^{1} \mathrm{H}$ NMR (300 MHz, $\left.\mathrm{CDCl}_{3}\right) \delta 7.89(\mathrm{~d}, \mathrm{~J}=8.2 \mathrm{~Hz}, 2 \mathrm{H}), 7.55(\mathrm{~d}, \mathrm{~J}=1.7 \mathrm{~Hz}, 2 \mathrm{H}), 7.34(\mathrm{dd}, \mathrm{J}=8.3$, 
$1.7 \mathrm{~Hz}, 2 \mathrm{H}), 4.28(\mathrm{q}, \mathrm{J}=7.2 \mathrm{~Hz}, 2 \mathrm{H}), 1.43(\mathrm{t}, \mathrm{J}=7.2 \mathrm{~Hz}, 3 \mathrm{H}) .{ }^{13} \mathrm{C}$ NMR $\left(75 \mathrm{MHz}, \mathrm{CDCl}_{3}\right) \delta$ $141.1(2 \mathrm{C}), 122.7(2 \mathrm{CH}), 121.7(2 \mathrm{CH}), 121.6(2 \mathrm{C}), 119.9(2 \mathrm{C}), 112.0(2 \mathrm{CH}), 38.0\left(\mathrm{CH}_{2}\right), 13.9$ $\left(\mathrm{CH}_{3}\right)$ ppm. IR (ATR platinum) 2971, 2932, 1869, 1621, 1584, 1485, 1471, 1449, 1424, 1404, 1377, 1347, 1339, 1326, 1315, 1268, 1231, 1223, 1132, 1084, 1055, 1024, 997, 941, 919, 890, $851,841,802,794,786,750,721,667,603,590,558,435 \mathrm{~cm}^{-1}$. HRMS (ASAP, $80^{\circ} \mathrm{C}$ ): $[\mathrm{M}+\mathrm{H}]^{+}$ found 351.9336 for $\mathrm{C}_{14} \mathrm{H}_{12} \mathrm{NBr}_{2}$ required 351.9331. m.p.: $133{ }^{\circ} \mathrm{C}$.

\section{2,7-dibromo-N-butyl-carbazole}

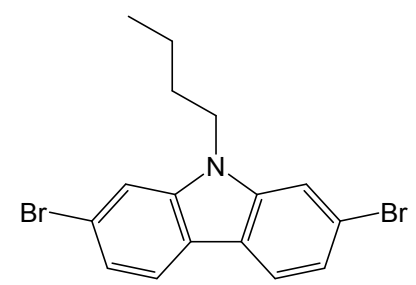

The title compound was synthesized using the general procedure for alkylation of 2,7-dibromo$9 \mathrm{H}$-carbazole.

2,7-Dibromo-9H-carbazole (2.50 g, $7.69 \mathrm{mmol}, 1.0 \mathrm{eq})$, KOH (1.29 g, $23.1 \mathrm{mmol}, 3.0 \mathrm{eq})$, tetrabutylammonium hydrogen sulfate $(0.261 \mathrm{mg}, 0.769 \mathrm{mmol}, 0.1 \mathrm{eq}), 1$-bromobutane $(1.79$ $\mathrm{mL}, 19.2 \mathrm{mmol}, 2.5 \mathrm{eq})$ and dry DMF (30 mL).

After purification flash chromatography on silica gel [column condition: silica cartridge ( $40 \mathrm{~g})$; solid deposit on Celite ${ }^{\circledR}$; $\lambda$ detection: $(254 \mathrm{~nm}, 280 \mathrm{~nm}) ; \mathrm{CH}_{2} \mathrm{Cl}_{2} /$ light petroleum (5/95) at 40 $\mathrm{mL} / \mathrm{min}]$, the title compound was obtained as a white solid $(2.85 \mathrm{~g}, 7.46 \mathrm{mmol})$; yield $97 \%$; ${ }^{1} \mathrm{H}$ $\operatorname{NMR}\left(300 \mathrm{MHz}, \mathrm{CDCl}_{3}\right) \delta 7.89(\mathrm{~d}, \mathrm{~J}=8.2 \mathrm{~Hz}, 2 \mathrm{H}), 7.53(\mathrm{~d}, \mathrm{~J}=1.7 \mathrm{~Hz}, 2 \mathrm{H}), 7.34(\mathrm{dd}, \mathrm{J}=8.3$, $1.7 \mathrm{~Hz}, 2 \mathrm{H}), 4.21(\mathrm{t}, \mathrm{J}=7.2 \mathrm{~Hz}, 2 \mathrm{H}), 1.84(\mathrm{tt}, \mathrm{J}=9.5,6.8 \mathrm{~Hz}, 2 \mathrm{H}), 1.48-1.33(\mathrm{~m}, 2 \mathrm{H}), 0.97(\mathrm{t}$, $\mathrm{J}=7.3 \mathrm{~Hz}, 3 \mathrm{H}) .{ }^{13} \mathrm{C}$ NMR $\left(75 \mathrm{MHz}, \mathrm{CDCl}_{3}\right) \delta 141.5(2 \mathrm{C}), 122.7(2 \mathrm{CH}), 121.6(2 \mathrm{CH}), 121.4$ (2C), $119.8(2 \mathrm{C}), 112.1(2 \mathrm{CH}), 43.3\left(\mathrm{CH}_{2}\right), 31.1\left(\mathrm{CH}_{2}\right), 20.7\left(\mathrm{CH}_{2}\right), 14.0\left(\mathrm{CH}_{3}\right) \mathrm{ppm}$. IR (ATR platinum) $2958,2926,2874,2858,1621,1585,1565,1483,1467,1452,1441,1424,1406$, 1382, 1371, 1341, 1327, 1316, 1266, 1241, 1225, 1212, 1203, 1133, 1117, 1054, 997, 976, 957, $938,899,878,852,835,815,793,771,750,733,721,665,649,592,558,436,422 \mathrm{~cm}^{-1}$. HRMS (ASAP, $130-{ }^{\circ} \mathrm{C}$ ): Found $[\mathrm{M}+\mathrm{H}]^{+}, 379.9648, \mathrm{C}_{16} \mathrm{H}_{16} \mathrm{NBr}_{2}$ required 379.9644. m.p.: $118^{\circ} \mathrm{C}$.

\section{2,7-dibromo-N-hexyl-carbazole}

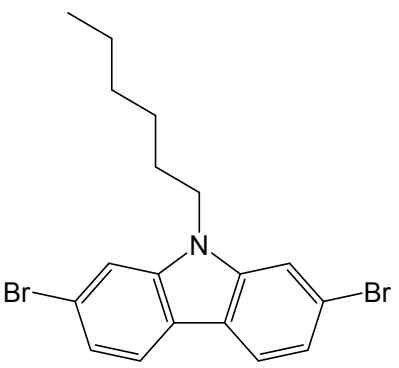

The title compound was synthesized using the general procedure for alkylation of 2,7-dibromo$9 \mathrm{H}$-carbazole.

2,7-Dibromo-9H-carbazole (3.00 g, $9.23 \mathrm{mmol}, 1.0 \mathrm{eq}), \mathrm{KOH}$ (1.55 g, $27.7 \mathrm{mmol}, 3.0 \mathrm{eq})$, tetrabutylammonium hydrogen sulfate $(0.313 \mathrm{mg}, 0.923 \mathrm{mmol}, 0.1 \mathrm{eq}), 1$-bromohexane $(3.23$ $\mathrm{mL}, 23.1 \mathrm{mmol}, 2.5 \mathrm{eq})$ and dry DMF $(30 \mathrm{~mL})$.

After purification flash chromatography on silica gel [column condition: silica cartridge ( $40 \mathrm{~g})$; solid deposit on Celite ${ }^{\circ}$; $\lambda$ detection: $\left(254 \mathrm{~nm}, 280 \mathrm{~nm}\right.$ ); $\mathrm{CH}_{2} \mathrm{Cl}_{2} /$ light petroleum (5/95) at 40 $\mathrm{mL} / \mathrm{min}]$, the title compound was obtained as a white solid $(3.53 \mathrm{~g}, 8.35 \mathrm{mmol})$; yield $90 \%$; ${ }^{1} \mathrm{H}$ $\operatorname{NMR}\left(300 \mathrm{MHz}, \mathrm{CDCl}_{3}\right): \delta 7.89(\mathrm{~d}, \mathrm{~J}=8.3 \mathrm{~Hz}, 2 \mathrm{H}), 7.53(\mathrm{~d}, \mathrm{~J}=1.7 \mathrm{~Hz}, 2 \mathrm{H}), 7.34(\mathrm{dd}, \mathrm{J}=8.3$, $1.7 \mathrm{~Hz}, 2 \mathrm{H}), 4.19$ (t, J = 7.4 Hz, 2H), $1.84(\mathrm{~m}, \mathrm{~J}=7.3 \mathrm{~Hz}, 2 \mathrm{H}), 1.46-1.22(\mathrm{~m}, 6 \mathrm{H}), 0.90(\mathrm{t}, 3 \mathrm{H})$ 
ppm. ${ }^{13} \mathrm{C}$ NMR (75 MHz, $\left.\mathrm{CDCl}_{3}\right) \delta 141.5(2 \mathrm{C}), 122.7(2 \mathrm{CH}), 121.6(2 \mathrm{CH}), 121.4(2 \mathrm{C}), 119.8$ (2C), $112.2(2 \mathrm{CH}), 43.5\left(\mathrm{CH}_{2}\right), 31.6\left(\mathrm{CH}_{2}\right), 28.9\left(\mathrm{CH}_{2}\right), 27.0\left(\mathrm{CH}_{2}\right), 22.7\left(\mathrm{CH}_{2}\right), 14.1\left(\mathrm{CH}_{3}\right)$ ppm. IR (ATR platinum) 2964, 2946, 2922, 2866, 2851, 1623, 1587, 1570, 1484, 1466, 1447, $1422,1409,1367,1347,1340,1327,1312,1300,1276,1268,1252,1234,1223,1193,1129$, 1053, 1043, 1026, 997, 922, 904, 873, 866, 833, 817, 793, 770, 750, 740, 722, 665, 650, 593, 558, 443, $425 \mathrm{~cm}^{-1}$. HRMS (ASAP, $90^{\circ} \mathrm{C}$ ): $\mathrm{M}^{+}$. Found 406.9878 for $\mathrm{C}_{18} \mathrm{H}_{19} \mathrm{~N}^{79} \mathrm{Br}_{2}$ required 406.98787. m.p.: $74^{\circ} \mathrm{C}$.

General procedure for the synthesis of bis(pinacolato) diboron carbazole derivatives The corresponding alkylated dibromocarbazole (1.0 eq) was dissolved in anhydrous THF $(0.03$ M) under an argon atmosphere. The reaction mixture was cooled to $-78^{\circ} \mathrm{C}$. A $2.5 \mathrm{M}$ in hexanes solution of $n-\mathrm{BuLi}(3.3 \mathrm{eq})$ was then added dropwise to the solution at $-78^{\circ} \mathrm{C}$. The solution was stirred at this temperature for one hour and 2-isopropoxy-4,4,5,5-tetramethyl-1,3,2,dioxaborolane $(3.5 \mathrm{eq})$ was added to the mixture and stirred at $-78^{\circ} \mathrm{C}$ for 15 minutes. The reaction mixture was allowed to warm up to room temperature overnight under stirring. The reaction mixture was quenched with few drops of absolute ethanol and concentrated under reduced pressure. The crude product was dissolved in $\mathrm{CH}_{2} \mathrm{Cl}_{2}$. The organic layer was washed with water, brine, dried over $\mathrm{MgSO}_{4}$, filtered and concentrated under reduced pressure. The crude was purified with flash chromatography on silica gel.

\section{N-ethyl-2,7-bis(4,4,5,5-tetramethyl-1,3,2-dioxaborolan-2-yl)-carbazole EtCbzBpin 2}

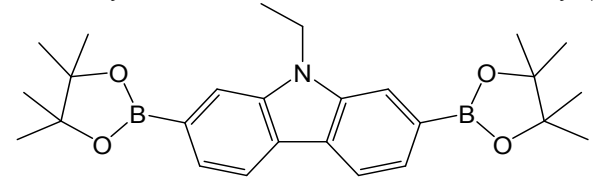

The title compound was synthesized using the general procedure for the synthesis of diboronic acid pinacol ester derivatives.

2,7-Dibromo- $N$-ethyl-carbazole (2.00 g, $5.66 \mathrm{mmol}, 1.0 \mathrm{eq})$, THF (120 mL), $n$-BuLi (7.47 mL, $18.68 \mathrm{mmol}, 3.3 \mathrm{eq})$ and 2-isopropoxy-4,4,5,5-tetramethyl-1,3,2,-dioxaborolane (4.04 mL, $19.81 \mathrm{mmol}, 3.5 \mathrm{eq})$.

After flash chromatography on silica gel [column conditions: silica cartridge (40 g); solid deposit on Celite ${ }^{\circledR}$; $\lambda$ detection: (254 nm, $\left.280 \mathrm{~nm}\right) ; \mathrm{CH}_{2} \mathrm{Cl}_{2} /$ light petroleum (3/7) at $40 \mathrm{~mL} / \mathrm{min}$ ], the title compound was obtained as a white solid (2.04 g, $4.56 \mathrm{mmol})$; yield 81\%; ${ }^{1} \mathrm{H}$ NMR (300 $\left.\mathrm{MHz} \mathrm{CDCl}_{3}\right) \delta 8.15(\mathrm{dd}, \mathrm{J}=7.8,0.8 \mathrm{~Hz}, 2 \mathrm{H}), 7.92(\mathrm{~s}, 2 \mathrm{H}), 7.71(\mathrm{dd}, \mathrm{J}=7.8,0.9 \mathrm{~Hz}, 2 \mathrm{H}), 4.49$ (q, J $=7.2 \mathrm{~Hz}, 2 \mathrm{H}), 1.48(\mathrm{t}, \mathrm{J}=7.2 \mathrm{~Hz}, 3 \mathrm{H}), 1.43(\mathrm{~s}, 24 \mathrm{H}) .{ }^{13} \mathrm{C} \mathrm{NMR}\left(75 \mathrm{MHz}, \mathrm{CDCl}_{3}\right) \delta 140.1$ (2C), $125.3(4 \mathrm{C}), 125.0(2 \mathrm{CH}), 120.2(2 \mathrm{CH}), 115.2(2 \mathrm{CH}), 84.0(4 \mathrm{C}), 37.6\left(\mathrm{CH}_{2}\right), 25.1\left(8 \mathrm{CH}_{3}\right)$, $14.4\left(\mathrm{CH}_{3}\right)$ ppm. IR (ATR platinum): 2991, 2977, 1560, 1501, 1483, 1453, 1436, 1390, 1381, 1370, 1357, 1338, 1316, 1261, 1233, 1211, 1167, 1142, 1132, 1111, 1076, 1058, 1000, 965, $949,937,876,854,836,818,776,757,736,706,688,671,646,595,579,559,421 \mathrm{~cm}^{-1}$. HRMS (ASAP, $240^{\circ} \mathrm{C}$ ): Found $[\mathrm{M}+\mathrm{H}]^{+}, 448.2832, \mathrm{C}_{26} \mathrm{H}_{36} \mathrm{NO}_{4}{ }^{11} \mathrm{~B}_{2}$ required 448.28249. m.p.: $342^{\circ} \mathrm{C}$.

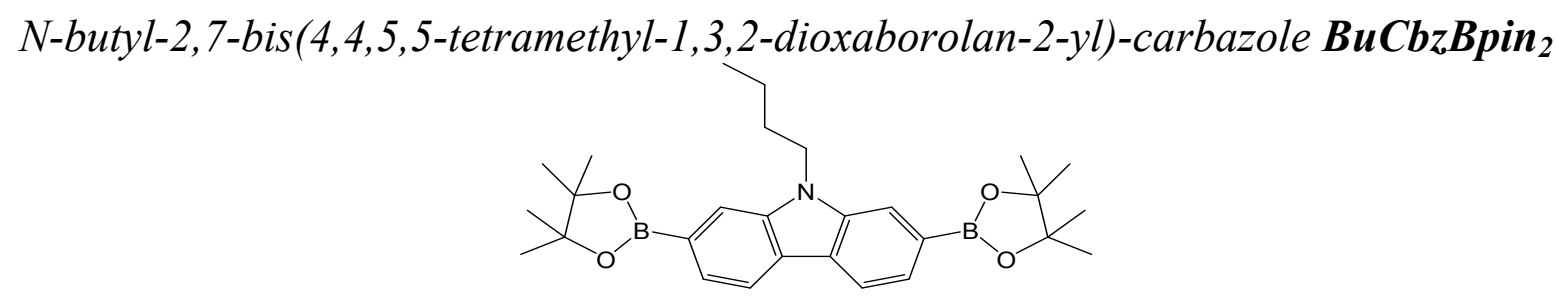


The title compound was synthesized using the general procedure for the synthesis of diboronic acid pinacol ester derivatives.

2,7-Dibromo- $N$-butyl-carbazole (2.00 g, $5.25 \mathrm{mmol}, 1.0 \mathrm{eq})$, THF ( $80 \mathrm{~mL}), n$-BuLi $(11.5 \mathrm{~mL}$, $17.3 \mathrm{mmol}, 3.3 \mathrm{eq})$ and 2-isopropoxy-4,4,5,5-tetramethyl-1,3,2,-dioxaborolane $(3.75 \mathrm{~mL}, 18.4$ mmol, 3.5 eq).

After flash chromatography on silica gel [column conditions: silica cartridge (40 g); solid deposit on Celite ${ }^{\circledR}$; $\lambda$ detection: (254 nm, $280 \mathrm{~nm}$ ); $\mathrm{CH}_{2} \mathrm{Cl}_{2} /$ light petroleum (3/7) at $40 \mathrm{~mL} / \mathrm{min}$ ], the title compound was obtained as a white solid (1.85 g, $3.89 \mathrm{mmol})$; yield $73 \%$; ${ }^{1} \mathrm{H}$ NMR $\left(300 \mathrm{MHz}, \mathrm{CDCl}_{3}\right): \delta 8.12(\mathrm{dd}, \mathrm{J}=7.8,0.8 \mathrm{~Hz}, 2 \mathrm{H}), 7.89(\mathrm{~s}, 2 \mathrm{H}), 7.68(\mathrm{dd}, \mathrm{J}=7.8,0.9 \mathrm{~Hz}, 2 \mathrm{H})$, $4.39(\mathrm{t}, \mathrm{J}=7.3 \mathrm{~Hz}, 2 \mathrm{H}), 1.97-1.80(\mathrm{~m}, 2 \mathrm{H}), 1.40(\mathrm{~s}, 26 \mathrm{H}), 0.95(\mathrm{t}, \mathrm{J}=7.3 \mathrm{~Hz}, 3 \mathrm{H}) .{ }^{13} \mathrm{C} \mathrm{NMR}$ $\left(75 \mathrm{MHz}, \mathrm{CDCl}_{3}\right) \delta 140.6(2 \mathrm{C}), 125.2(4 \mathrm{C}), 125.0(2 \mathrm{CH}), 120.2(2 \mathrm{CH}), 115.4(2 \mathrm{CH}), 83.9(8 \mathrm{C})$, $42.9\left(\mathrm{CH}_{2}\right), 31.6\left(\mathrm{CH}_{2}\right), 25.1\left(8 \mathrm{CH}_{3}\right), 20.7\left(\mathrm{CH}_{2}\right), 14.1\left(\mathrm{CH}_{3}\right)$ ppm. IR (ATR, platinum): 2979 , 2956, 2928, 1624, 1558, 1499, 1482, 1466, 1450, 1433, 1391, 1381, 1371, 1354, 1337, 1313, $1266,1249,1231,1213,1167,1141,1113,1075,1056,998,972,958,914,880,856,824,808$, $782,735,706,689,673,644,579,558,521,420 \mathrm{~cm}^{-1}$. HRMS (ASAP, $170{ }^{\circ} \mathrm{C}$ ): Found $[\mathrm{M}+\mathrm{H}]^{+}$, 476.3143, $\mathrm{C}_{28} \mathrm{H}_{40} \mathrm{NO}_{4}{ }^{11} \mathrm{~B}_{2}$ required 476.31379. m.p. : $213^{\circ} \mathrm{C}$.

N-hexyl-2,7-bis(4,4,5,5-tetramethyl-1,3,2-dioxaborolan-2-yl)-carbazole HexCbzBpin

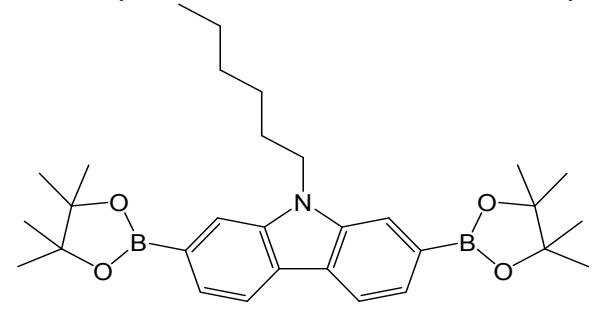

The title compound was synthesized using the general procedure for the synthesis of diboronic acid pinacol ester derivatives.

2,7-Dibromo- $N$-butyl-carbazole (2.00 g, $4.89 \mathrm{mmol}, 1.0 \mathrm{eq})$, THF ( $80 \mathrm{~mL}), n$-BuLi (6.45 mL, $16.1 \mathrm{mmol}, 3.3 \mathrm{eq})$ and 2-isopropoxy-4,4,5,5-tetramethyl-1,3,2,-dioxaborolane (3.49 mL, 17.1 mmol, $3.5 \mathrm{eq})$.

After flash chromatography on silica gel [column conditions: silica cartridge (40 g); solid deposit on Celite ${ }^{\circledR}$; $\lambda$ detection: $(254 \mathrm{~nm}, 280 \mathrm{~nm}) ; \mathrm{CH}_{2} \mathrm{Cl}_{2} /$ light petroleum (3/7) at $40 \mathrm{~mL} / \mathrm{min}$ ], giving the title compound as a white solid $(2.17 \mathrm{~g}, 4.30 \mathrm{mmol})$; yield $88 \%$; ${ }^{1} \mathrm{H} \mathrm{NMR} \mathrm{(300} \mathrm{MHz,}$ $\left.\mathrm{CDCl}_{3}\right): \delta 8.14(\mathrm{~d}, \mathrm{~J}=7.8 \mathrm{~Hz}, 2 \mathrm{H}), 7.91(\mathrm{~s}, 2 \mathrm{H}), 7.70(\mathrm{~d}, \mathrm{~J}=7.8 \mathrm{~Hz}, 2 \mathrm{H}), 4.41(\mathrm{t}, \mathrm{J}=7.3 \mathrm{~Hz}$, $2 \mathrm{H}), 1.91(\mathrm{~m}, \mathrm{~J}=7.3 \mathrm{~Hz}, 2 \mathrm{H}), 1.43(\mathrm{~s}, 30 \mathrm{H}), 0.91(\mathrm{t}, \mathrm{J}=7.0 \mathrm{~Hz}, 3 \mathrm{H}) .{ }^{13} \mathrm{C} \mathrm{NMR}(75 \mathrm{MHz}$, $\left.\mathrm{CDCl}_{3}\right) \delta 140.6(2 \mathrm{C}), 125.2(4 \mathrm{C}), 125.0(2 \mathrm{CH}), 120.2(2 \mathrm{CH}), 115.4(2 \mathrm{CH}), 83.9(8 \mathrm{C}), 43.0$ $\left(\mathrm{CH}_{2}\right), 31.8\left(\mathrm{CH}_{2}\right), 29.3\left(\mathrm{CH}_{2}\right), 27.0\left(\mathrm{CH}_{2}\right), 25.1\left(8 \mathrm{CH}_{3}\right), 22.8\left(\mathrm{CH}_{2}\right), 14.2\left(\mathrm{CH}_{3}\right) \mathrm{ppm}$. IR (ATR platinum): 2994, 2977, 2956, 2926, 2861, 1625, 1599, 1560, 1501, 1484, 1454, 1433, 1392, 1380, 1371, 1355, 1338, 1312, 1267, 1256, 1236, 1213, 1166, 1141, 1113, 1076, 1060, 999, 966, 940, 909, 882, 855, 822, 790, 764, 736, 728, 706, 689, 671, 642, 590, 577, 557, 520, 419 $\mathrm{cm}^{-1}$. HRMS (ESI, $\mathrm{CH}_{3} \mathrm{OH} / \mathrm{CH}_{2} \mathrm{Cl}_{2}$ : 90/10): $[\mathrm{M}+\mathrm{K}]^{+}$found 542.3010 for $\mathrm{C}_{30} \mathrm{H}_{43} \mathrm{NO}_{4}{ }^{11} \mathrm{~B}_{2} \mathrm{~K}$ required 542.30098. m.p.: $209^{\circ} \mathrm{C}$. 
General procedure for nanoring synthesis

Diboronic acid pinacol ester derivative $(1.0 \mathrm{eq})$, cesium fluoride $(6.0 \mathrm{eq})$ and 1,5cyclooctadieneplatinum (II) dichloride (1.0 eq) were dissolved in solvent A $\left(2.65 \times 10^{-2}\right.$ mol. L $^{-}$ 1) under an argon atmosphere and stirred at $70^{\circ} \mathrm{C}$ for $24 \mathrm{~h}$. Solvent was then removed under reduced pressure and the crude product was dried under vacuum at $60^{\circ} \mathrm{C}$ for $24 \mathrm{~h}$. Triphenylphosphine (15 eq) was then added to the crude product and Solvent B $\left(1.77 \times 10^{-2}\right.$ mol.L $L^{-1}$ ) was added under an argon atmosphere, see Table S 1 . The resulting mixture was stirred for one or half an hour at room temperature and then at the reflux of solvent B for $48 \mathrm{~h}$. Then, solvent was removed under reduced pressure. The crude product was dissolved in $\mathrm{CH}_{2} \mathrm{Cl}_{2}$. Water was added and the organic layer was extracted with $\mathrm{CH}_{2} \mathrm{Cl}_{2}$, dried over $\mathrm{MgSO}_{4}$, filtered and solvent was removed under reduced pressure.

Table S 1 Optimization of solvents system for transmetallation and reductive elimination step in nanorings synthesis.

\begin{tabular}{|c|c|c|c|c|}
\hline \multirow{2}{*}{ Nanoring } & \multirow{2}{*}{$\begin{array}{l}\text { Transmetallation } \\
\text { Solvent A }\end{array}$} & \multicolumn{2}{|c|}{ reductive elimination } & \multirow{2}{*}{ Yield $(\%)$} \\
\hline & & Solvent B & Time (h) & \\
\hline [4]C-Et-Cbz & THF & Toluene & $0.5 / 24$ & 6 \\
\hline [4]C-Et-Cbz & THF & $o$-DCB & $0.5 / 24$ & 16 \\
\hline [4]C-Et-Cbz & THF & $o$-DCB & $1 / 48$ & 25 \\
\hline [4]C-Et-Cbz & DCE & $o-\mathrm{DCB}$ & $1 / 48$ & 64 \\
\hline [4]C-Bu-Cbz & THF & $o$-DCB & $1 / 48$ & 25 \\
\hline [4]C-Bu-Cbz & $\mathrm{DCE}$ & $o-\mathrm{DCB}$ & $1 / 48$ & 60 \\
\hline$[4] \mathrm{C}-\mathrm{Bu}-\mathrm{Cbz}$ & TCE & $o$-DCB & $1 / 48$ & $<1$ \\
\hline [4]C-Bu-Cbz & $\mathrm{CCl}_{4}$ & $o$-DCB & $1 / 48$ & $<1$ \\
\hline [4]C-Bu-Cbz & DMF & $o$-DCB & $1 / 48$ & 9 \\
\hline [4]C-Bu-Cbz & $o-\mathrm{DCB}$ & $o-\mathrm{DCB}$ & $1 / 48$ & $<1$ \\
\hline [4]C-Hex-Cbz & THF & $o$-DCB & $1 / 48$ & 24 \\
\hline [4]C-Hex-Cbz & DCE & $o$-DCB & $1 / 48$ & 52 \\
\hline
\end{tabular}

[4]cyclo-N-ethyl-2,7-carbazole [4]C-Et-Cbz

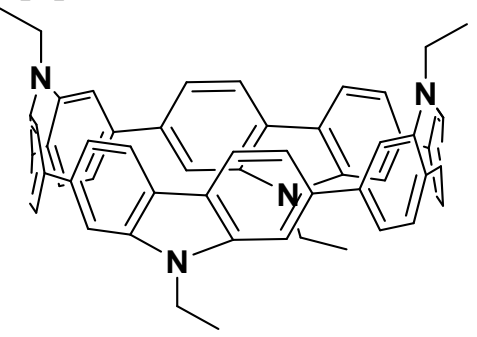

The title compound was synthesized using the general procedure for nanoring synthesis.

$1^{\text {st }}$ step: 3a $(0.250 \mathrm{~g}, 0.559 \mathrm{mmol}, 1.0 \mathrm{eq})$, cesium fluoride $(0.510 \mathrm{~g}, 5.35 \mathrm{mmol}, 9.6 \mathrm{eq})$ and 1,5-cyclooctadieneplatinum (II) dichloride $(0.209 \mathrm{~g}, 0.559 \mathrm{mmol}, 1.0 \mathrm{eq})$ in extra dry DCE (21 $\mathrm{mL})$. 
$2^{\text {nd }}$ step: Triphenylphosphine $(2.20 \mathrm{~g}, 8.39 \mathrm{mmol}, 15 \mathrm{eq})$ was then added to the crude product in 1,2-dichlorobenzene (32 mL).

The residue was purified first with flash chromatography on silica gel [column conditions: silica cartridge $(80 \mathrm{~g})$; solid deposit on Celite ${ }^{\circledR} ; \lambda$ detection $(254 \mathrm{~nm}, 280 \mathrm{~nm})$; gradient dichloromethane/light petroleum $20 \%$ to $50 \%$ for $60 \mathrm{~min}$ at $40 \mathrm{~mL} / \mathrm{min}$ ] and second with recycling size exclusion chromatography $\left(\mathrm{CHCl}_{3}\right)$ giving the title compound as a yellow solid (69 mg; 8.93×10-2 mmol) yield $64 \% ;{ }^{1} \mathrm{H}$ NMR $\left(300 \mathrm{MHz}, \mathrm{CDCl}_{3}\right) \delta 7.95(\mathrm{~d}, \mathrm{~J}=8.3 \mathrm{~Hz}, 8 \mathrm{H})$, $7.51(\mathrm{dd}, \mathrm{J}=8.3,1.4 \mathrm{~Hz}, 8 \mathrm{H}), 6.53(\mathrm{~d}, \mathrm{~J}=1.4 \mathrm{~Hz}, 8 \mathrm{H}), 3.71(\mathrm{q}, \mathrm{J}=7.2 \mathrm{~Hz}, 8 \mathrm{H}), 1.19$ (t, J = 7.2 $\mathrm{Hz}, 12 \mathrm{H}) ;{ }^{13} \mathrm{C}$ NMR $\left(75 \mathrm{MHz}, \mathrm{CD}_{2} \mathrm{Cl}_{2}\right) \delta 143.95(8 \mathrm{C}), 141.93(16 \mathrm{C}), 122.69(8 \mathrm{CH}), 117.03$ $(8 \mathrm{CH}), 115.88(8 \mathrm{CH}), 37.37\left(4 \mathrm{CH}_{2}\right), 14.39\left(4 \mathrm{CH}_{3}\right) \mathrm{ppm}$. IR (ATR platinum): 3049, 2968, 2931, $1610,1588,1560,1474,1447,1415,1380,1326,1318,1259,1241,1226,1175,1129,1074$, 1060, 1014, 994, 939, 937, 868, 849; 824, 792, 753, 735, 654, 628, 584, 552, 480, $458 \mathrm{~cm}^{-1}$; HRMS (ESI $\left.{ }^{+}, \mathrm{CH}_{2} \mathrm{Cl}_{2}\right)$ : Found $\left([\mathrm{M}]^{+}, 772.3556 ; \mathrm{C}_{56} \mathrm{H}_{44} \mathrm{~N}_{4}\right.$ required $772.35605 ;$ m.p. $>350^{\circ} \mathrm{C}$

\section{[4]cyclo-N-butyl-2,7-carbazole [4]C-Bu-Cbz}

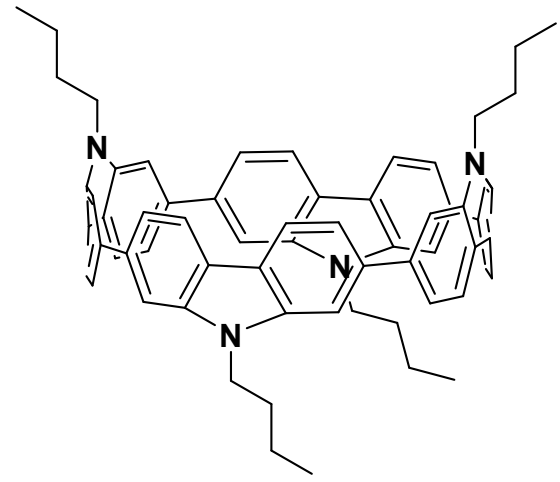

The title compound was synthesized using the general procedure for nanoring synthesis.

$1^{\text {st }}$ step : 3b (0.250 g, $\left.0.526 \mathrm{mmol}, 1.0 \mathrm{eq}\right)$, cesium fluoride $(0.479 \mathrm{~g}, 3.16 \mathrm{mmol}, 6.0 \mathrm{eq})$ and 1,5-cyclooctadieneplatinum (II) dichloride $(0.197 \mathrm{~g}, 0.526 \mathrm{mmol}, 1.0 \mathrm{eq})$ in extra dry DCE (20 $\mathrm{mL})$.

$2^{\text {nd }}$ step: Triphenylphosphine $(2.07 \mathrm{~g}, 7.89 \mathrm{mmol}, 15 \mathrm{eq})$ was then added to the crude product in 1,2-dichlorobenzene $(30 \mathrm{~mL})$.

The residue was purified first with flash chromatography on silica gel [column conditions: silica cartridge (80 g); solid deposit on Celite ${ }^{\circledR}$; $\lambda$ detection $(254 \mathrm{~nm}, 280 \mathrm{~nm})$; gradient dichloromethane/light petroleum $20 \%$ to $50 \%$ for $60 \mathrm{~min}$ at $40 \mathrm{~mL} / \mathrm{min}$ ] and second with recycling size exclusion chromatography $\left(\mathrm{CHCl}_{3}\right)$ to give the title compound as a yellow solid $\left(0.070 \mathrm{~g}, 7.91 \times 10^{-2} \mathrm{mmol}\right)$; yield $60 \%$; ${ }^{1} \mathrm{H}$ NMR $\left(300 \mathrm{MHz}, \mathrm{CDCl}_{3}\right) \delta 7.94(\mathrm{~d}, J=8.3 \mathrm{~Hz}$, $8 \mathrm{H}), 7.49(\mathrm{~d}, J=8.3 \mathrm{~Hz}, 8 \mathrm{H}), 6.49(\mathrm{~s}, 8 \mathrm{H}), 3.55(\mathrm{t}, J=6.9 \mathrm{~Hz}, 8 \mathrm{H}), 1.68(\mathrm{~m}, J=7.1 \mathrm{~Hz}, 8 \mathrm{H})$, $1.31(\mathrm{~m}, J=7.3 \mathrm{~Hz}, 8 \mathrm{H}), 0.89(\mathrm{t}, J=7.3 \mathrm{~Hz}, 12 \mathrm{H}) \mathrm{ppm} .{ }^{13} \mathrm{C} \mathrm{NMR}\left(75 \mathrm{MHz}, \mathrm{CDCl}_{3}\right) \delta 143.8$ (8C), $142.0(16 \mathrm{C}), 122.3(8 \mathrm{CH}), 116.7(8 \mathrm{CH}), 115.7(8 \mathrm{CH}), 41.8\left(4 \mathrm{CH}_{2}\right), 31.4\left(4 \mathrm{CH}_{2}\right), 20.3$ $\left(4 \mathrm{CH}_{2}\right), 13.9\left(4 \mathrm{CH}_{3}\right) \mathrm{ppm}$. IR(ATR platinum) 2957, 2929, 2899, 2869, 1610, 1587, 1560, 1542, $1475,1450,1414,1356,1320,1302,1257,1244,1217,1192,1131,1110,1066,1030,995$, $926,863,845,831,812,792,755,741,725,715,702,667,654,627,604,593,575,552,502$, 465, 452, 441, $424 \mathrm{~cm}^{-1}$. HRMS (MALDI, DCTB): $\mathrm{M}^{+}$. found 884.482 for $\mathrm{C}_{64} \mathrm{H}_{60} \mathrm{~N}_{4}$ required 884.48125. m.p. $>350^{\circ} \mathrm{C}$. 


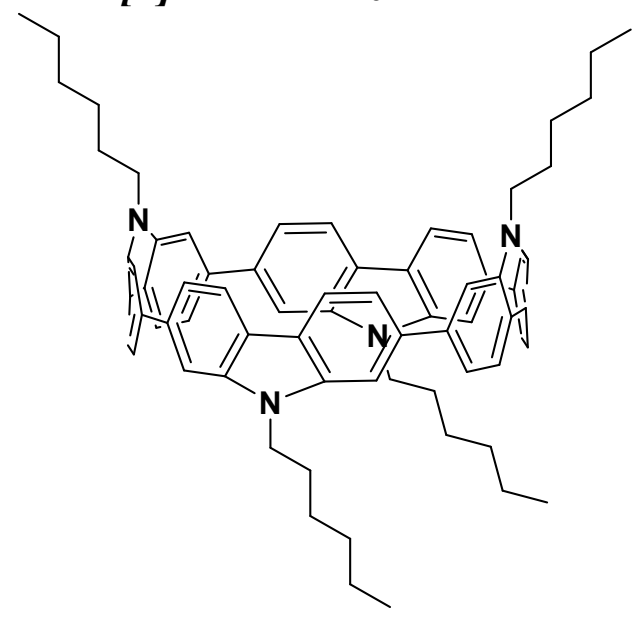

The title compound was synthesized using the general procedure for nanoring synthesis.

$1^{\text {st }}$ step: 3c $(0.250 \mathrm{~g}, 0.497 \mathrm{mmol}, 1.0 \mathrm{eq})$, cesium fluoride $(0.453 \mathrm{~g}, 2.98 \mathrm{mmol}, 6.0 \mathrm{eq})$ and 1,5-cyclooctadieneplatinum (II) dichloride $(0.186 \mathrm{~g}, 0.497 \mathrm{mmol}, 1.0 \mathrm{eq})$ in extra dry DCE (19 $\mathrm{mL})$.

$2^{\text {nd }}$ step: Triphenylphosphine ( $\left.1.95 \mathrm{~g}, 7.45 \mathrm{mmol}, 15 \mathrm{eq}\right)$ was then added to the crude product in 1,2-dichlorobenzene $(28 \mathrm{~mL})$.

The residue was purified first with flash chromatography on silica gel [column conditions: silica cartridge $(80 \mathrm{~g})$; solid deposit on Celite ${ }^{R} ; \lambda$ detection $(254 \mathrm{~nm}, 280 \mathrm{~nm})$; gradient dichloromethane/light petroleum $20 \%$ to $50 \%$ for $60 \mathrm{~min}$ at $40 \mathrm{~mL} / \mathrm{min}$ ] and second with recycling size exclusion chromatography $\left(\mathrm{CHCl}_{3}\right)$ giving the title compound as a yellow solid $\left(0.064 \mathrm{~g}, 6.42 \times 10^{-2} \mathrm{mmol}\right)$; yield $52 \% ;{ }^{1} \mathrm{H} \mathrm{NMR}\left(300 \mathrm{MHz}, \mathrm{CDCl}_{3}\right) \delta 7.93(\mathrm{~d}, J=8.3 \mathrm{~Hz}$, $8 \mathrm{H}), 7.49(\mathrm{~d}, J=8.3 \mathrm{~Hz}, 8 \mathrm{H}), 6.49(\mathrm{~s}, 8 \mathrm{H}), 3.54(\mathrm{t}, J=7.0 \mathrm{~Hz}, 6 \mathrm{H}), 1.64(\mathrm{q}, J=6.9 \mathrm{~Hz}, 6 \mathrm{H})$, $1.31-1.17(\mathrm{~m}, 24 \mathrm{H}), 0.86(\mathrm{t}, J=5.5 \mathrm{~Hz}, 12 \mathrm{H}) \mathrm{ppm} .{ }^{13} \mathrm{C} \mathrm{NMR}\left(75 \mathrm{MHz}, \mathrm{CDCl}_{3}\right) \delta 143.8(8 \mathrm{C})$, $142.0(16 \mathrm{C}), 122.3(8 \mathrm{CH}), 116.6(8 \mathrm{CH}), 115.7(8 \mathrm{CH}), 42.1\left(4 \mathrm{CH}_{2}\right), 31.5\left(4 \mathrm{CH}_{2}\right), 29.1\left(4 \mathrm{CH}_{2}\right)$, $26.7\left(4 \mathrm{CH}_{2}\right), 22.6\left(4 \mathrm{CH}_{2}\right), 14.2\left(4 \mathrm{CH}_{3}\right) \mathrm{ppm}$. IR( ATR platinum) : 2955, 2922, 2854, 1610 , $1587,1562,1550,1476,1450,1414,1376,1353,1319,1302,1258,1244,1219,1179,1130$, $1063,1028,995,863,847,822,798,758,745,725,654,625,552,458 \mathrm{~cm}^{-1}$. HRMS (ASAP, $330{ }^{\circ} \mathrm{C}$ ): Found $\left[\mathrm{M}^{+} \cdot\right], 996.6061, \mathrm{C}_{72} \mathrm{H}_{76} \mathrm{~N}_{4}$ required $996.60645 . \mathrm{m} . \mathrm{p} .>350^{\circ} \mathrm{C}$. 
3 2D COSY and NOESY NMR Spectra

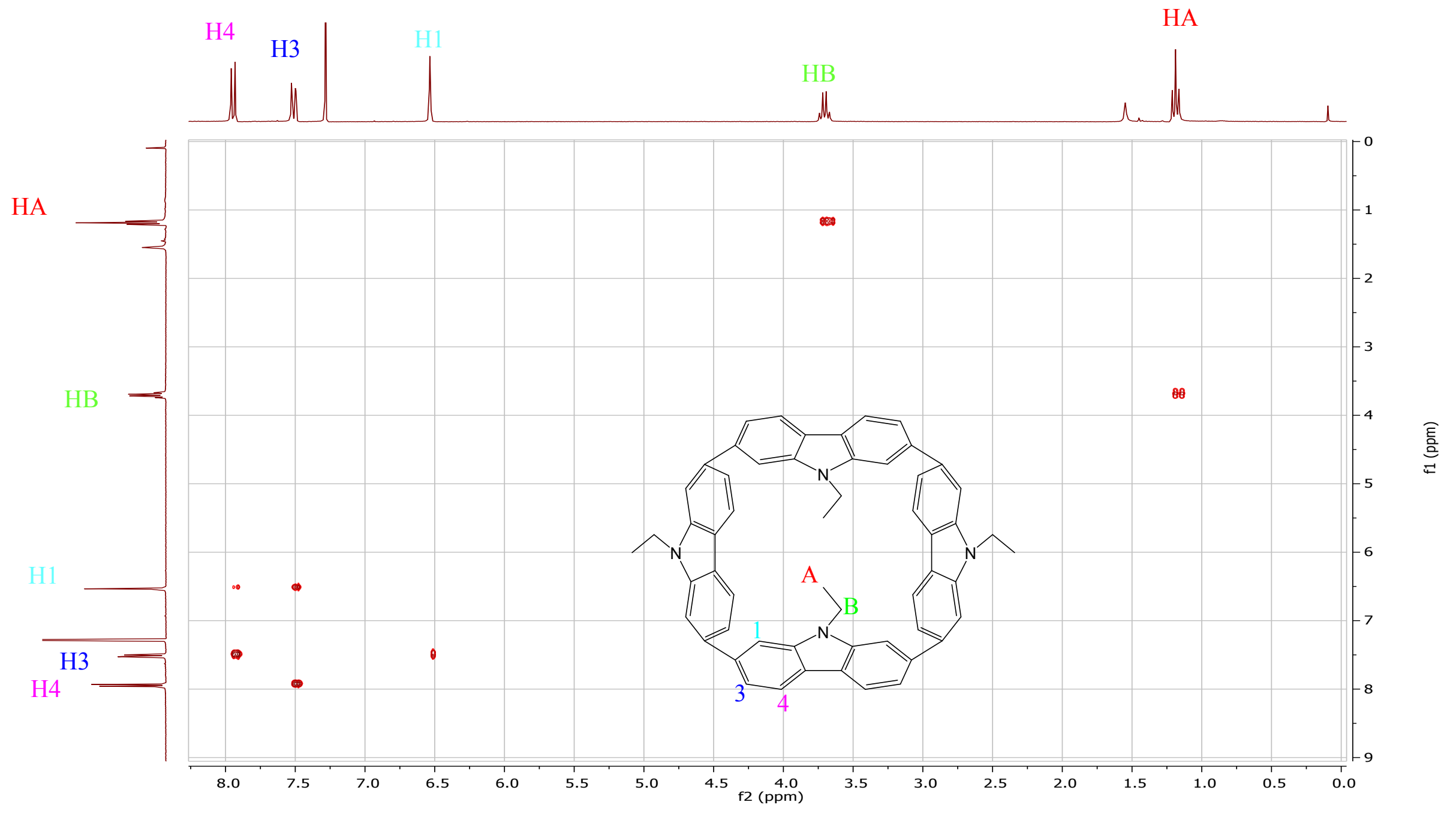

Figure $\mathrm{S} 1$ [4]C-Et-Cbz-COSY$-\mathrm{CDCl}_{3}$ 


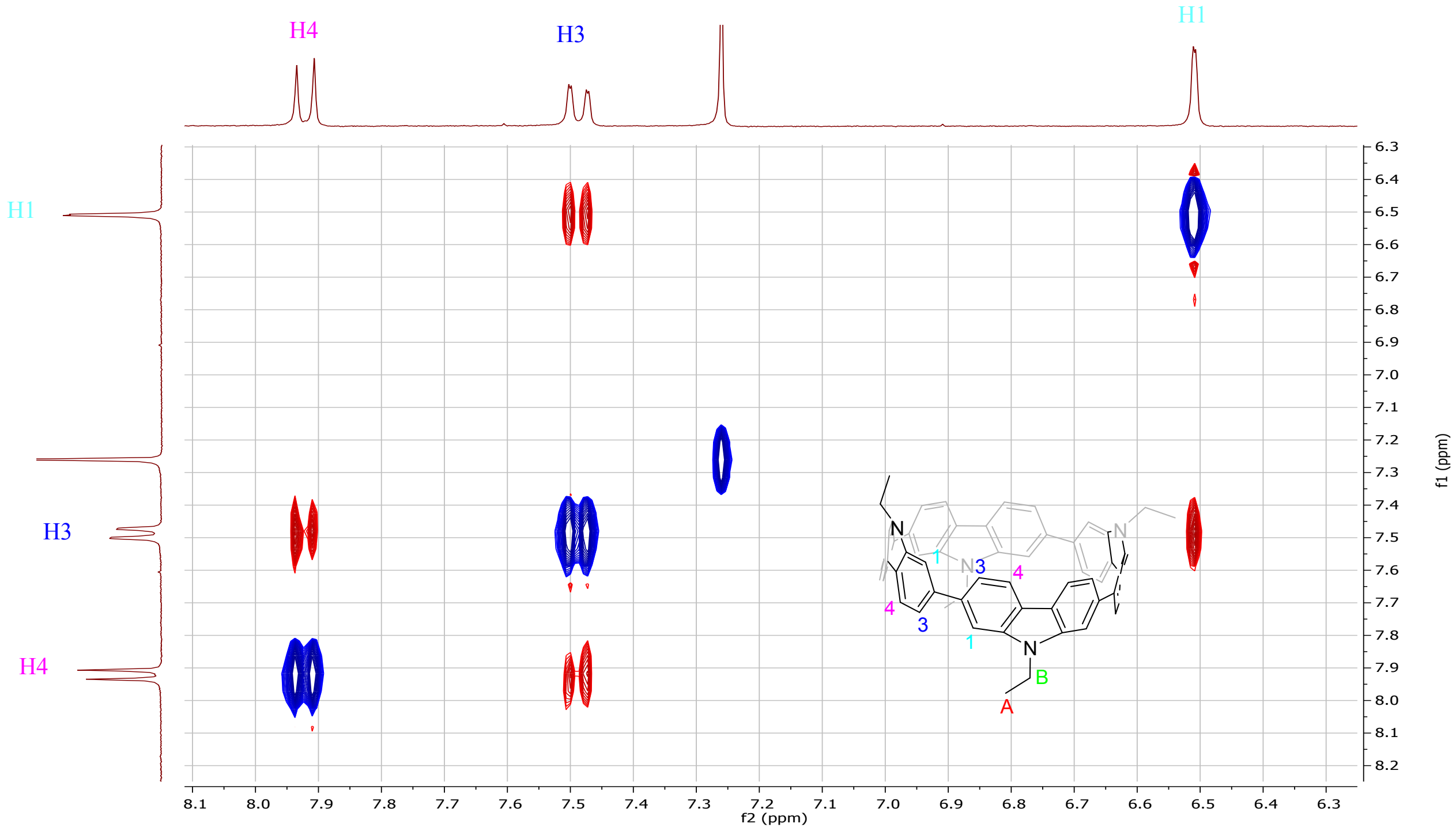

Figure $\mathrm{S} 2$ [4]C-Et-Cbz$-\mathrm{NOESY}-\mathrm{CDCl}_{3}$

14 


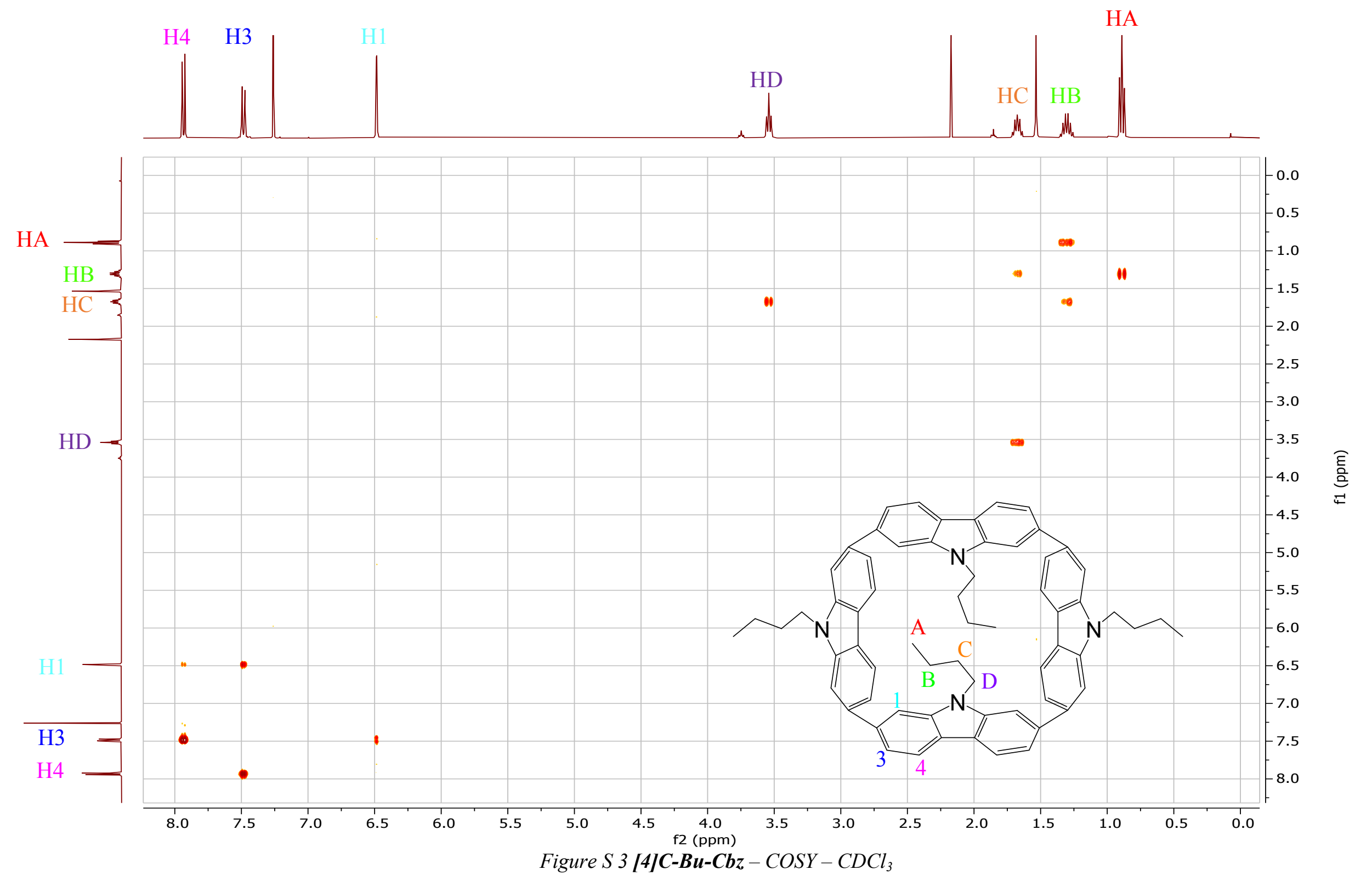




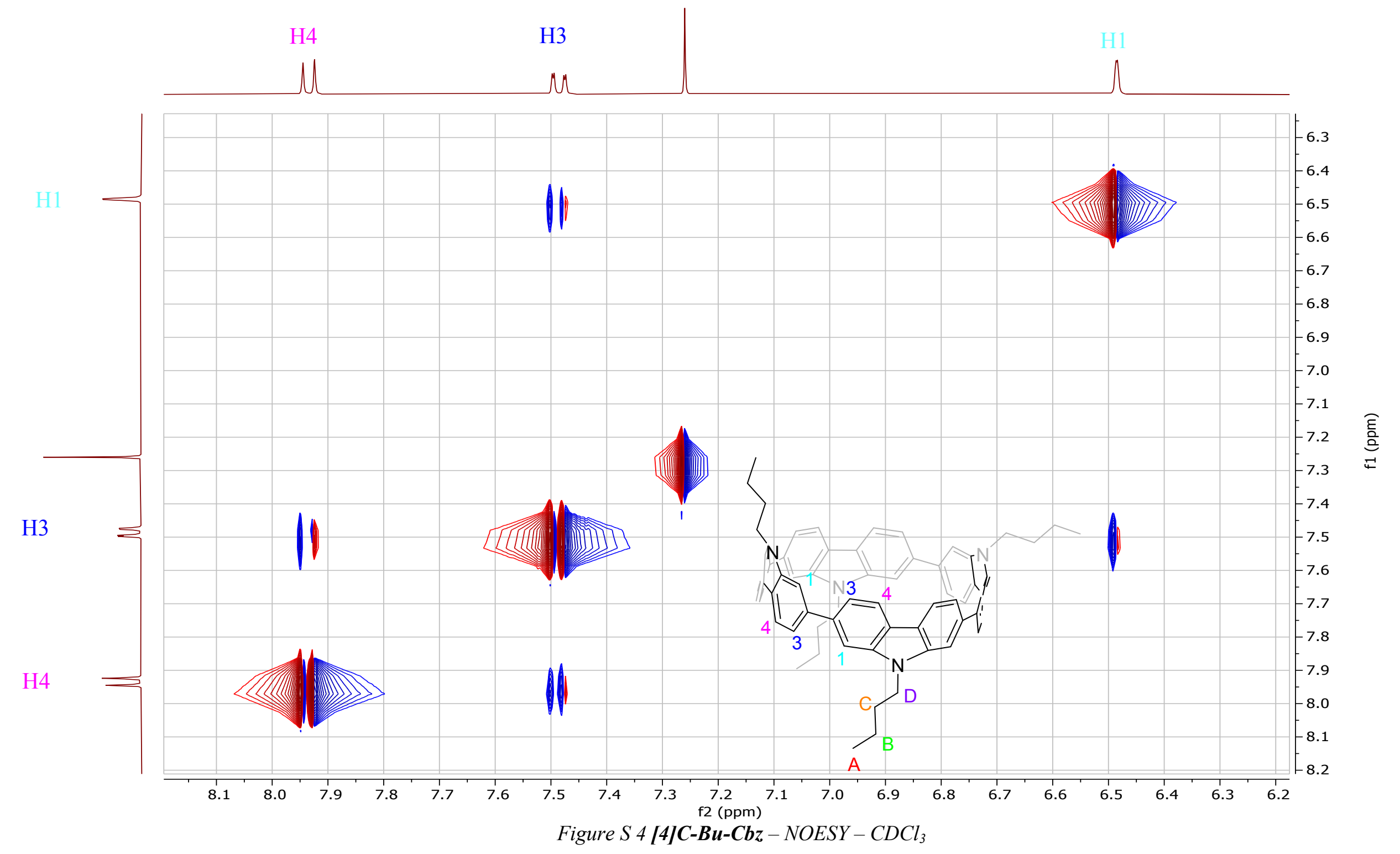




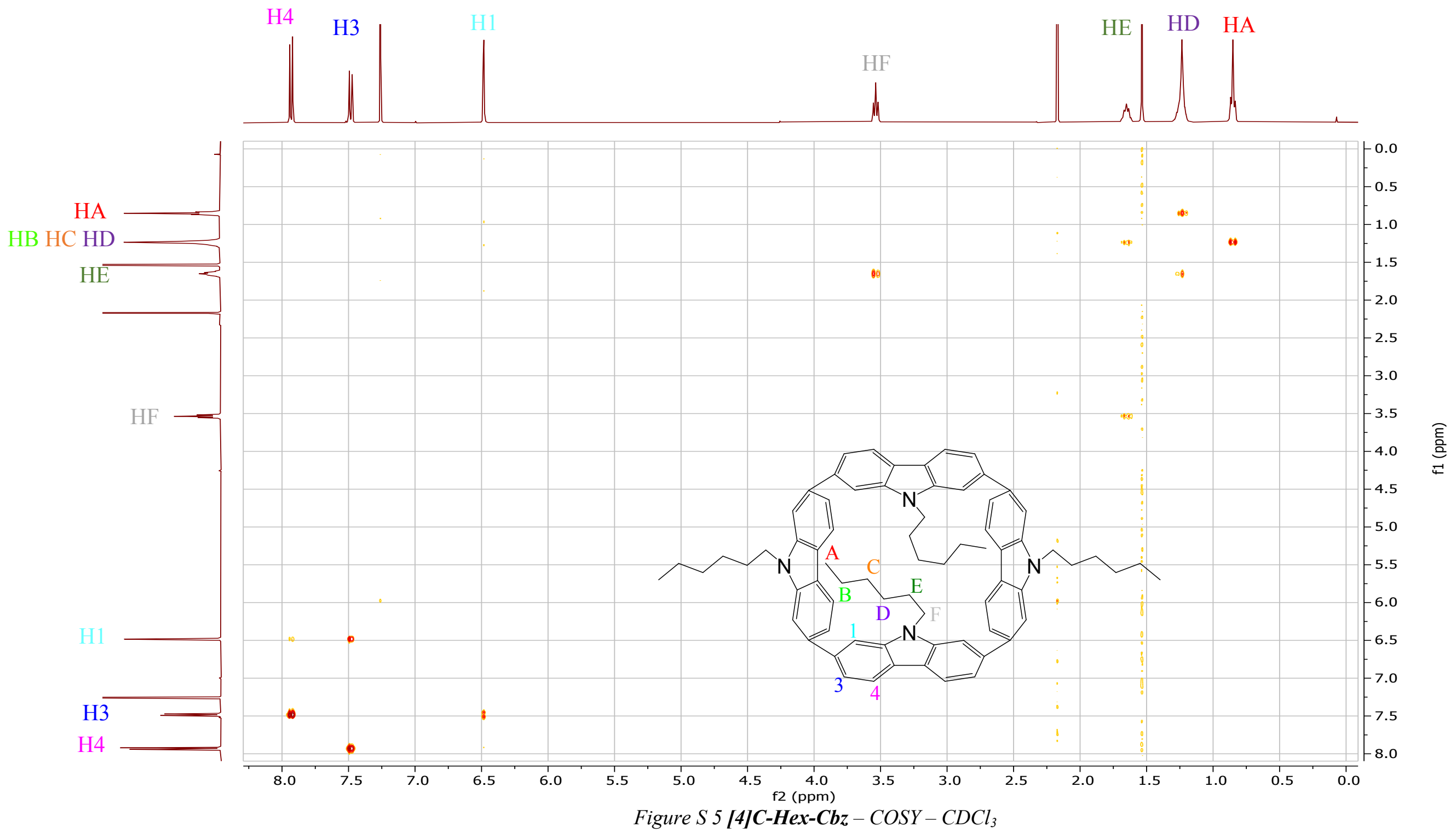




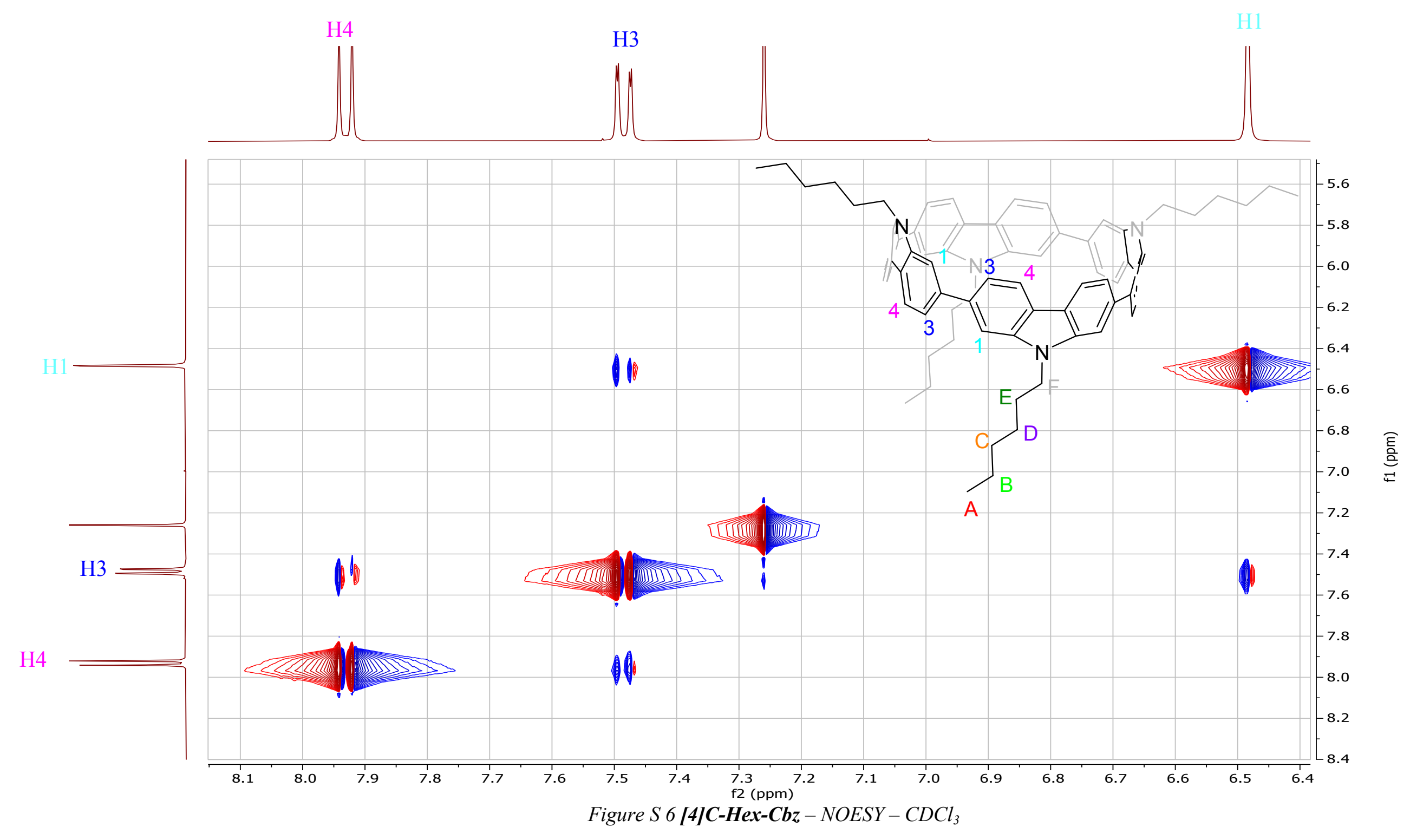




\section{Structural and thermal properties}

Axes and mean diameter $\varnothing$

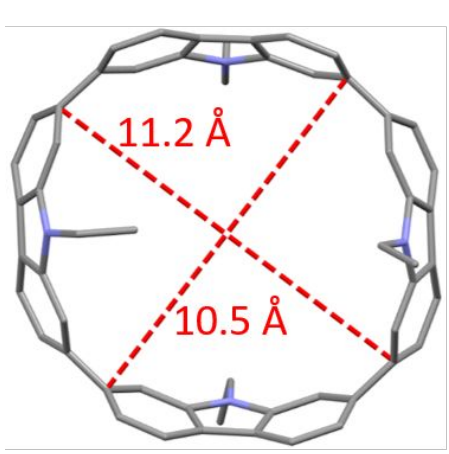

[4]C-Et-Cbz

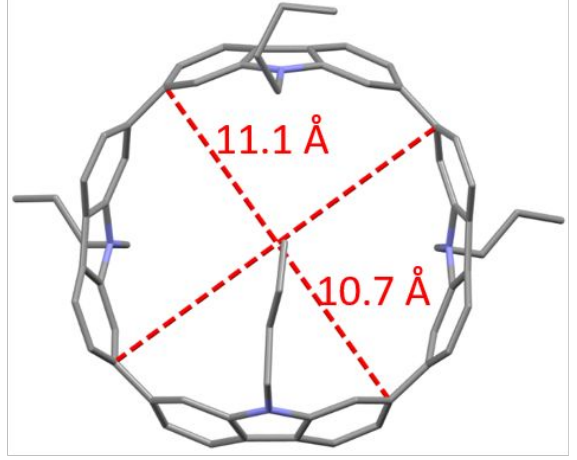

[4]C-Bu-Cbz

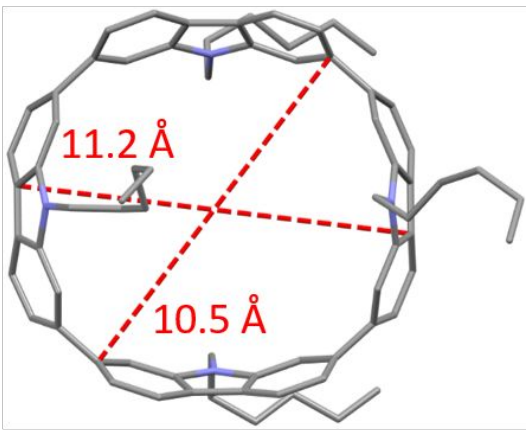

[4]C-Hex-Cbz

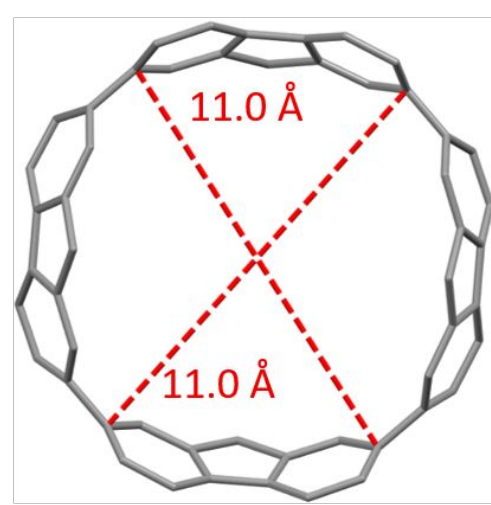

[4]C-diEt-F

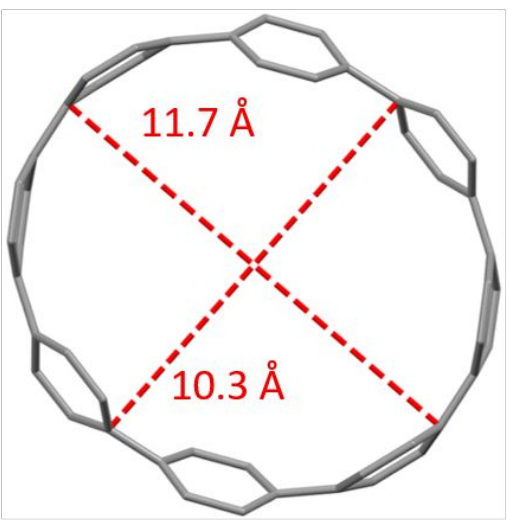

[8]CPP

Figure $S 7$ Minimal and maximal C-C axes in [4]C-Et-Cbz [4]C-Bu-Cbz, [4]C-Hex-Cbz, [4]C-diEt-F and $[8] \mathrm{CPP}$.

Table $S 2$ Axes and mean diameter values

\begin{tabular}{|c|c|c|}
\hline & $\operatorname{Axes}(\AA)$ & Mean diameter $\varnothing(\AA)$ \\
\hline \multirow{8}{*}[4]{$\mathrm{C}-\mathrm{Et}-\mathrm{Cbz}$} & 10.60 & \multirow{8}{*}{10.82} \\
\hline & 10.51 & \\
\hline & 10.49 & \\
\hline & 10.64 & \\
\hline & 11.10 & \\
\hline & 11.07 & \\
\hline & 11.19 & \\
\hline & 10.94 & \\
\hline \multirow{7}{*}[4]{$\mathrm{C}-\mathrm{Bu}-\mathrm{Cbz}$} & 10.75 & \multirow{7}{*}{10.86} \\
\hline & 10.71 & \\
\hline & 10.74 & \\
\hline & 11.12 & \\
\hline & 11.12 & \\
\hline & 10.99 & \\
\hline & 10.86 & \\
\hline
\end{tabular}




\begin{tabular}{|c|c|c|}
\hline & 10.89 & \\
\hline \multirow{8}{*}{ [4]C-Hex-Cbz } & 10.51 & \multirow{8}{*}{10.90} \\
\hline & 10.51 & \\
\hline & 10.86 & \\
\hline & 10.86 & \\
\hline & 11.06 & \\
\hline & 11.06 & \\
\hline & 11.18 & \\
\hline & 11.18 & \\
\hline \multirow{8}{*}{ [4]C-diEt-F } & 10.98 & \multirow{8}{*}{10.98} \\
\hline & 10.98 & \\
\hline & 10.98 & \\
\hline & 10.98 & \\
\hline & 10.98 & \\
\hline & 10.98 & \\
\hline & 10.98 & \\
\hline & 10.98 & \\
\hline \multirow{8}{*}[8]{$\mathrm{CPP}$} & 10.87 & \multirow{8}{*}{11.06} \\
\hline & 10.34 & \\
\hline & 10.93 & \\
\hline & 10.38 & \\
\hline & 11.34 & \\
\hline & 11.25 & \\
\hline & 11.65 & \\
\hline & 11.68 & \\
\hline
\end{tabular}

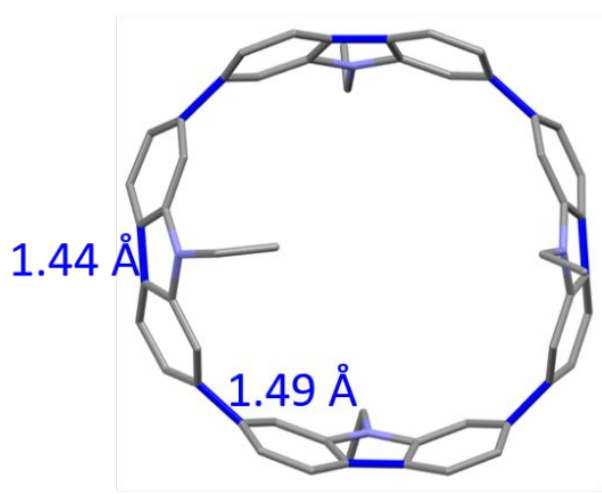

[4]C-Et-Cbz

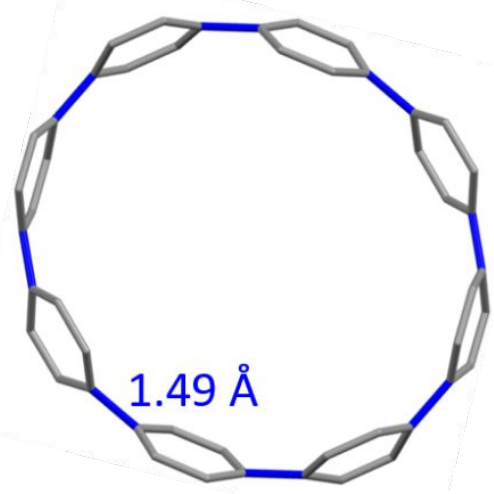

[8]CPP

Figure S 8 Average biphenyl bonds in [4]C-Et-Cbz and [8]CPP 


\section{Torsion angle}

Definition of a torsion angle: The external torsion angle $\left(\theta_{\text {ext }}\right)$ is the dihedral angle between two carbazole building units. Two angles are measured for each $\mathrm{C} 2-\mathrm{C} 7$ link (C8-C7-C2-C3 and C6$\mathrm{C} 7-\mathrm{C} 2-\mathrm{C} 1)$. The internal torsion angles $\left(\theta_{\text {int }}\right)$ are the dihedral angles within a building unit $(\mathrm{C} 4-$ $\mathrm{C} \beta-\mathrm{C} \gamma-\mathrm{C} 5$ and $\mathrm{C} \alpha-\mathrm{C} \beta-\mathrm{C} \gamma-\mathrm{C} \delta$ ).

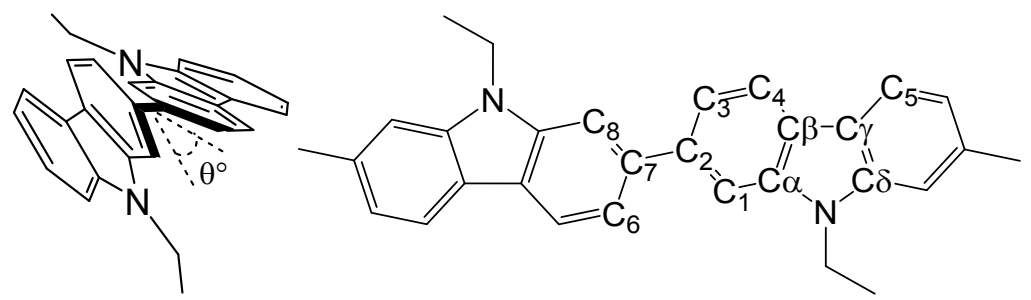

Figure S9 Definition of the torsion angle between two carbazole units

Table S3 Torsion angle values

\begin{tabular}{|c|c|c|c|c|c|}
\hline & \multicolumn{2}{|c|}{$\square$ ext } & \multicolumn{2}{|c|}{$\square$ int } & \multirow{2}{*}{$\theta \square$} \\
\hline & Values & Average & Values & Average & \\
\hline \multirow{8}{*}[4]{$\mathrm{C}-\mathrm{Et}-\mathrm{Cbz}$} & 38.7 & \multirow{8}{*}{40.6} & 0.09 & \multirow{8}{*}{0.8} & \multirow{8}{*}{20.7} \\
\hline & 41.2 & & 0.2 & & \\
\hline & 40.7 & & 2.2 & & \\
\hline & 40.4 & & 2.9 & & \\
\hline & 42.4 & & 0.1 & & \\
\hline & 40.7 & & 0.02 & & \\
\hline & 40.4 & & 0.01 & & \\
\hline & 40.5 & & 0.7 & & \\
\hline \multirow{8}{*}[4]{$\mathrm{C}-\mathrm{Bu}-\mathrm{Cbz}$} & 39.5 & \multirow{8}{*}{37.7} & 0.1 & \multirow{8}{*}{0.3} & \multirow{8}{*}{19.0} \\
\hline & 41.3 & & 0.2 & & \\
\hline & 36.8 & & 0.7 & & \\
\hline & 34.6 & & 0.9 & & \\
\hline & 35.7 & & 0.02 & & \\
\hline & 36.6 & & 0.2 & & \\
\hline & 37.8 & & 0.5 & & \\
\hline & 39.3 & & 0.1 & & \\
\hline \multirow{8}{*}{ [4]C-Hex-Cbz } & 41.1 & \multirow{8}{*}{41.1} & 0.0 & \multirow{8}{*}{0.5} & \multirow{8}{*}{20.3} \\
\hline & 43.2 & & 0.0 & & \\
\hline & 40.4 & & 0.4 & & \\
\hline & 39.7 & & 1.8 & & \\
\hline & 40.4 & & 0.0 & & \\
\hline & 39.7 & & 0.0 & & \\
\hline & 43.2 & & 0.4 & & \\
\hline & 41.1 & & 1.8 & & \\
\hline \multirow{6}{*}[8]{$\mathrm{CPP}$} & 42.5 & \multirow{6}{*}{24.3} & & & \multirow{6}{*}{24.3} \\
\hline & 40.4 & & & & \\
\hline & 24.9 & & & & \\
\hline & 23.1 & & & & \\
\hline & 1.5 & & & & \\
\hline & 1.6 & & & & \\
\hline
\end{tabular}




\begin{tabular}{|c|c|c|c|c|c|}
\hline & 31.3 & & & & \\
\hline & 29.4 & & & & \\
\hline & 42.5 & & & & \\
\hline & 40.4 & & & & \\
\hline & 24.9 & & & & \\
\hline & 23.1 & & & & \\
\hline & 1.5 & & & & \\
\hline & 1.6 & & & & \\
\hline & 31.3 & & & & \\
\hline & 29.4 & & & & \\
\hline \multirow{8}{*}{ [4]C-diEt-F } & 41.5 & \multirow{8}{*}{41.5} & 0.0 & \multirow{8}{*}{0.0} & \multirow{8}{*}{20.8} \\
\hline & 41.5 & & 0.0 & & \\
\hline & 41.5 & & 0.0 & & \\
\hline & 41.5 & & 0.0 & & \\
\hline & 41.5 & & 0.0 & & \\
\hline & 41.5 & & 0.0 & & \\
\hline & 41.5 & & 0.0 & & \\
\hline & 41.5 & & 0.0 & & \\
\hline
\end{tabular}

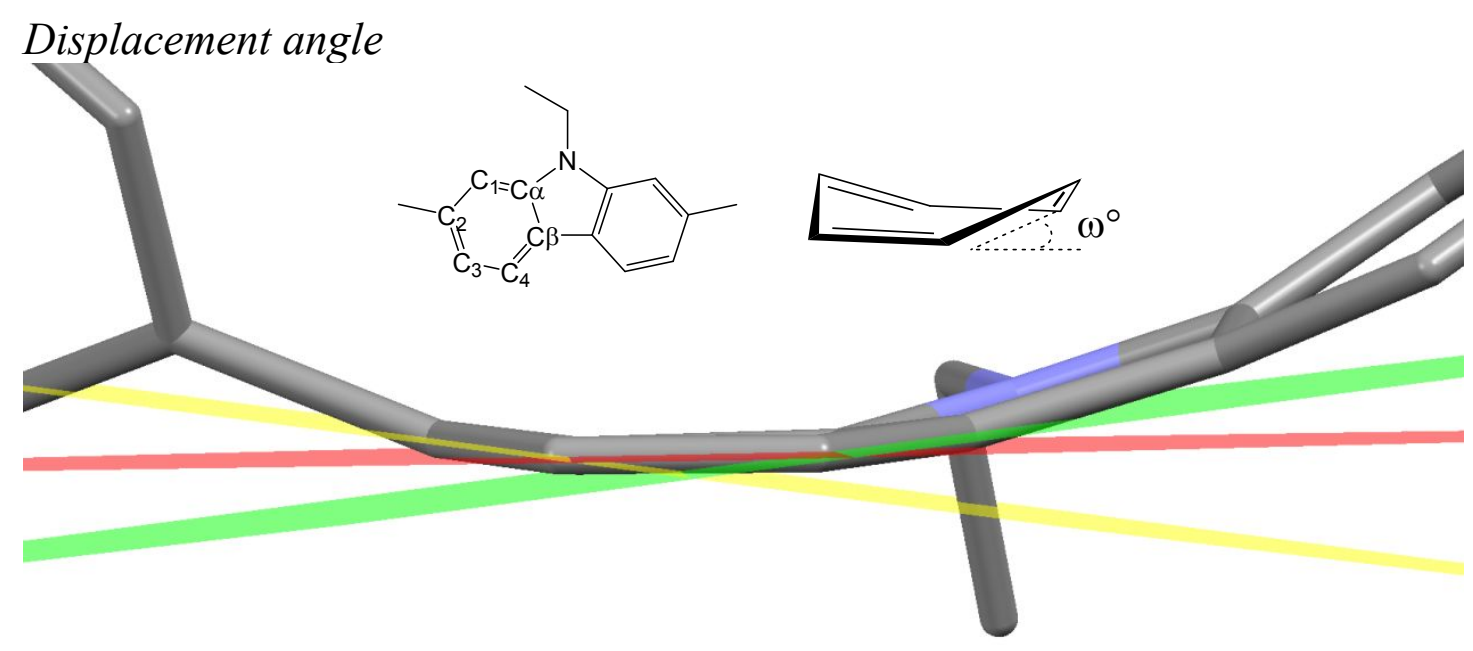

Figure S10 Measurement of a displacement angle

The displacement angle can represent the deformation of a phenyl unit.

Definition of a displacement angle: The mean planes passing by $\mathrm{C} \alpha-\mathrm{C} 1-\mathrm{C} 3-\mathrm{C} 4$ (red), $\mathrm{C} 1-\mathrm{C} 2-$ $\mathrm{C} 3$ (yellow) and $\mathrm{C} \alpha-\mathrm{C} \beta-\mathrm{C} 4$ (green) are drawn. The external displacement angle $\left(\omega_{\mathrm{ext}}\right)$ is measured between red and yellow planes, and, the internal displacement angle $\left(\omega_{\text {int }}\right)$ between red and green planes. Then, the mean displacement angle is calculated taking into account two $\omega_{\text {ext }}$ and two $\omega_{\text {int }}$ for each carbazole building units. 
Table S4 Displacement angle values

\begin{tabular}{|c|c|c|c|c|c|}
\hline & \multicolumn{2}{|c|}{$\omega e x t$} & \multicolumn{2}{|c|}{$\omega$ int } & \multirow{2}{*}{$\omega$} \\
\hline & Values & Average & Values & Average & \\
\hline \multirow{8}{*}{ [4]C-Et-Cbz } & $8.4^{\circ}$ & \multirow{8}{*}{7.9} & $6.1^{\circ}$ & \multirow{8}{*}{6.6} & \multirow{8}{*}{7.2} \\
\hline & $7.3^{\circ}$ & & $6.4^{\circ}$ & & \\
\hline & $8.4^{\circ}$ & & $7.8^{\circ}$ & & \\
\hline & $8.0^{\circ}$ & & $6.3^{\circ}$ & & \\
\hline & $6.9^{\circ}$ & & $6.2^{\circ}$ & & \\
\hline & $8.1^{\circ}$ & & $6.2^{\circ}$ & & \\
\hline & $8.5^{\circ}$ & & $6.1^{\circ}$ & & \\
\hline & $7.4^{\circ}$ & & $7.3^{\circ}$ & & \\
\hline \multirow{8}{*}[4]{$\mathrm{C}-\mathrm{Bu}-\mathrm{Cbz}$} & 7.4 & \multirow{8}{*}{7.8} & 5.3 & \multirow{8}{*}{6.3} & \multirow{8}{*}{7.1} \\
\hline & 7.2 & & 6.2 & & \\
\hline & 8.9 & & 7.6 & & \\
\hline & 7.4 & & 5.1 & & \\
\hline & 7.6 & & 6.5 & & \\
\hline & 8.0 & & 7.2 & & \\
\hline & 7.8 & & 6.1 & & \\
\hline & 8.2 & & 6.7 & & \\
\hline \multirow{8}{*}{ [4]C-Hex-Cbz } & 8.3 & \multirow{8}{*}{7.6} & 7.0 & \multirow{8}{*}{6.4} & \multirow{8}{*}{7.0} \\
\hline & 8.3 & & 7.0 & & \\
\hline & 6.8 & & 5.2 & & \\
\hline & 8.0 & & 6.3 & & \\
\hline & 7.5 & & 7.2 & & \\
\hline & 7.5 & & 7.2 & & \\
\hline & 8.0 & & 6.3 & & \\
\hline & 6.8 & & 5.2 & & \\
\hline \multirow{16}{*}[8]{$\mathrm{CPP}$} & 10.8 & & & & \multirow{16}{*}{9.6} \\
\hline & 11.4 & & & & \\
\hline & 8.1 & & & & \\
\hline & 8.3 & & & & \\
\hline & 7.7 & & & & \\
\hline & 7.2 & & & & \\
\hline & 11.1 & & & & \\
\hline & 12.1 & & & & \\
\hline & 10.8 & & & & \\
\hline & 11.4 & & & & \\
\hline & 8.1 & & & & \\
\hline & 8.3 & & & & \\
\hline & 7.7 & & & & \\
\hline & 7.2 & & & & \\
\hline & 11.1 & & & & \\
\hline & 12.1 & & & & \\
\hline \multirow{5}{*}{ [4]C-diEt-F } & 10.7 & \multirow{5}{*}{6.4} & 10.3 & \multirow{5}{*}{7.4} & \multirow{5}{*}{6.9} \\
\hline & 5.8 & & 5.7 & & \\
\hline & 6.1 & & 5.9 & & \\
\hline & 9.9 & & 9.1 & & \\
\hline & 7.7 & & 6.4 & & \\
\hline
\end{tabular}




\begin{tabular}{|l|l|l|l|l|l|}
\hline & 7.1 & \multirow{4}{*}{} & 6.1 & \multirow{4}{*}{} & \multirow{2}{*}{} \\
\cline { 2 - 2 } & 4.3 & 4.4 & \\
\cline { 2 - 2 } & 9.2 & 8.7 & \\
\hline
\end{tabular}
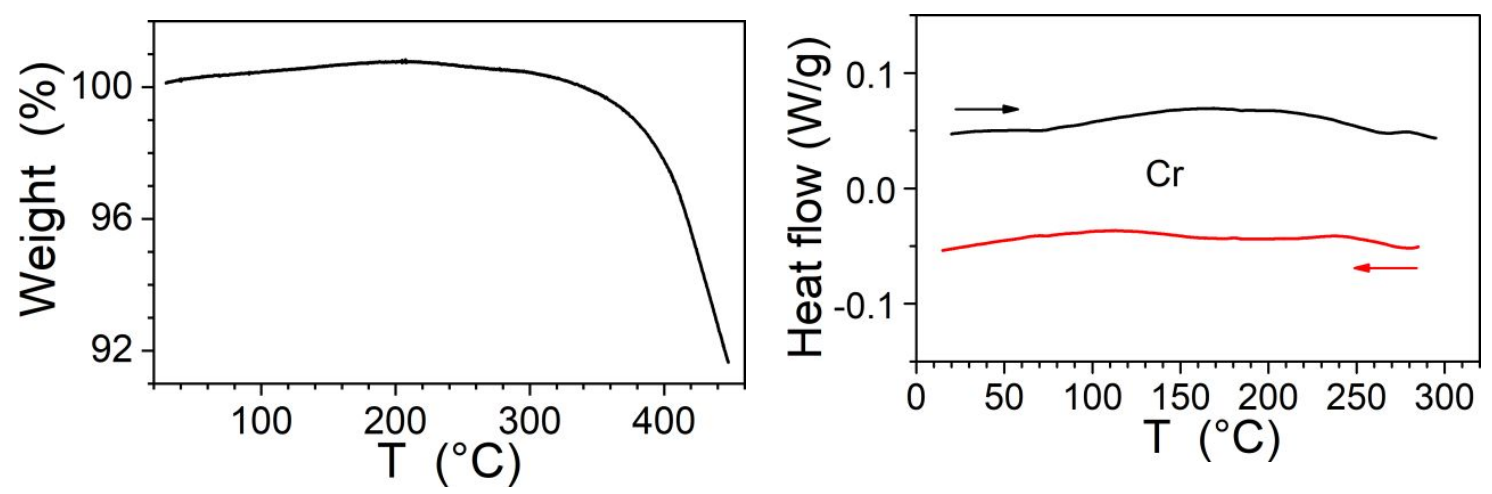

Figure S 11 left: TGA curves (Q50 from TA Instruments; conditions : $5^{\circ} \mathrm{C} / \mathrm{min}$, air) of [8]CPP. Sample amount was insufficient to obtain appropriate baseline, but results are enough to demonstrate that the weight loss from degradation starts around $350^{\circ} \mathrm{C}$ and reaches $5 \%$ at $T_{5 \%}=420^{\circ} \mathrm{C}$. Right: DSC curves on heating and on cooling (DSC Q1000 from TA Instruments; conditions: $5^{\circ} \mathrm{C} / \mathrm{min}$, endotherm up) that do not display any phase transition peak.
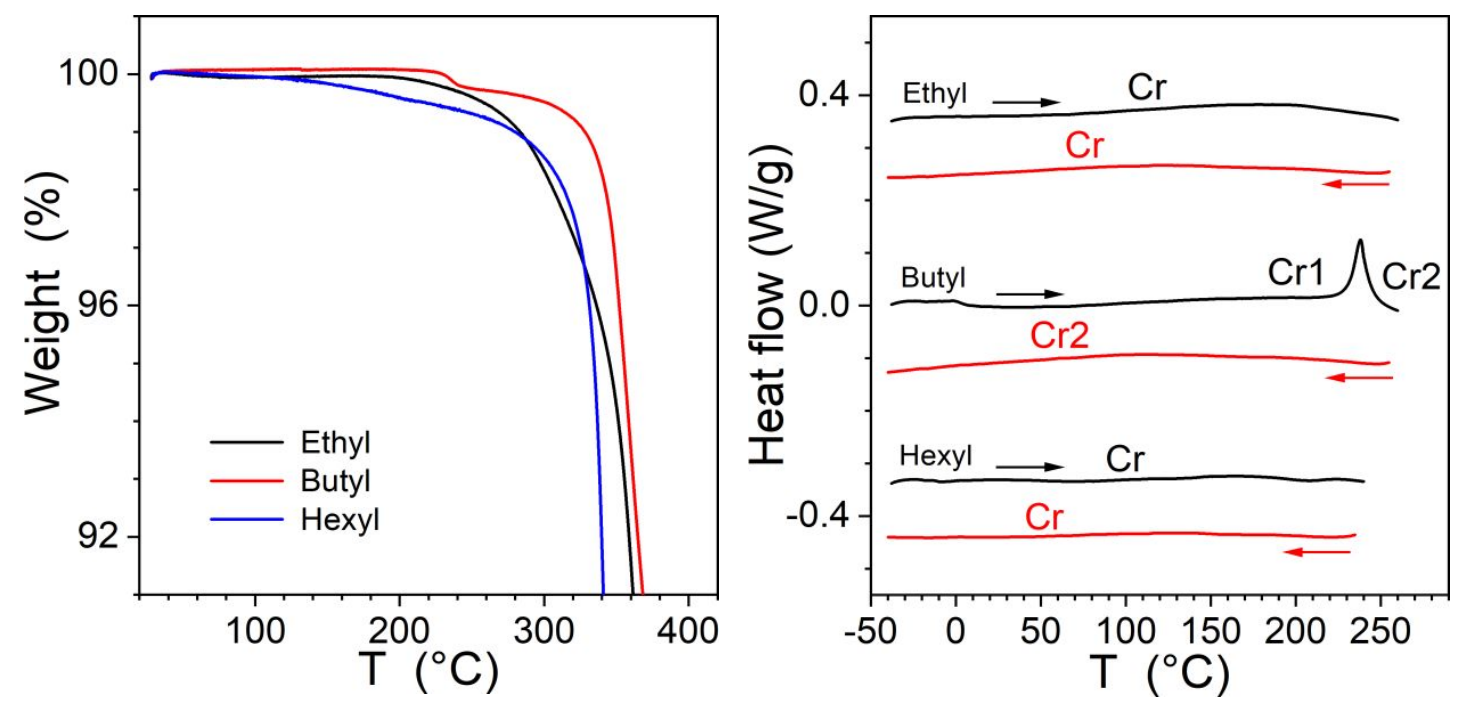

Figure S 12 Left: TGA curves $\left(5^{\circ} \mathrm{C} / \mathrm{min}\right.$, air). Right: DSC curves on first heating and on cooling (conditions: $5^{\circ} \mathrm{C} / \mathrm{min}$, endotherm up) and identification based on polarizing optical microscopy (POM) observations of the nanoring series 


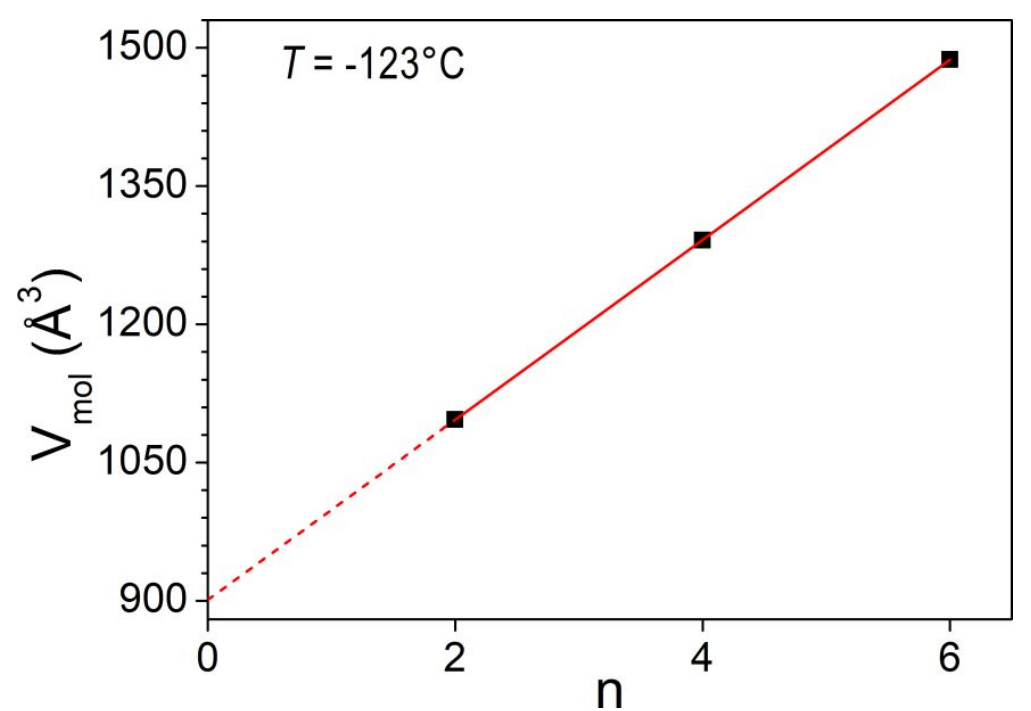

Figure $S 13$ Variation of molecular volume as a function of alkyl chain length (n=number of carbon atoms in the chain) in [4]C-R-Cbz single-crystal structures.

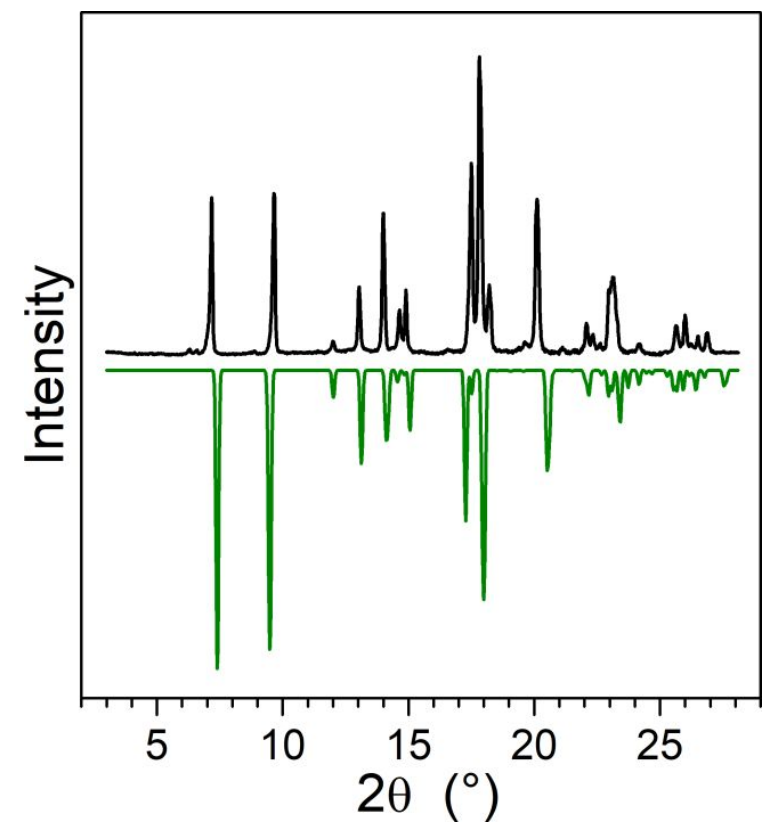

Figure $S 14$ Powder XRD patterns of $8 C P P$ powder at $20^{\circ} \mathrm{C}$ and pattern simulated from single-crystal structure CSD-KEBKAL 

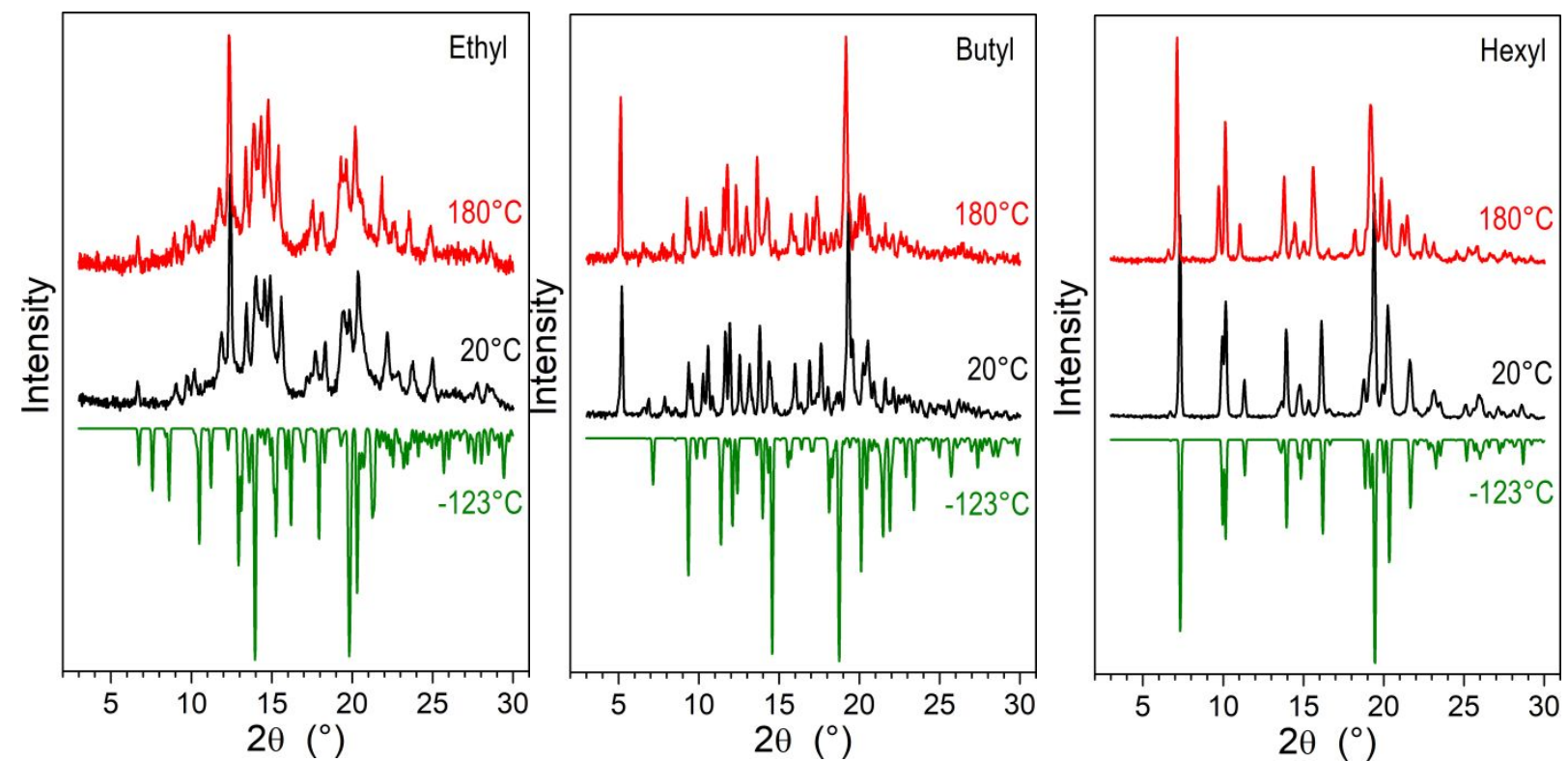

Figure S 15 Small- and wide-angle scattering (S/WAXS) patterns of [4]C-Et-Cbz (Left), [4]C-Bu-Cbz (Middle) and [4]C-Hex-Cbz (Right) at $20^{\circ} \mathrm{C}$ and $180^{\circ} \mathrm{C}$ (black and red lines), as compared to simulated powder XRD pattern from single crystal structures acquired at $-123^{\circ} \mathrm{C}$ (green line).

Table $S 5$ Cell parameters and cell compacity at temperature of measurement $T . V, \rho, Z, V / Z$ : cell volume, density, number of molecules per cell and cell volume per molecule; $V_{\text {solv }}$ : solvent volume calculated from density database; $V_{\text {mol }}=V / Z-V_{\text {solv }}$ : molecular volume.

\begin{tabular}{|c|c|c|c|c|c|c|}
\hline Compound & $\mathbf{T}\left[{ }^{\circ} \mathbf{C}\right]$ & $\begin{array}{l}\text { Cell parameters } \\
{\left[\AA ̊, \stackrel{\circ}{ } \AA^{3}\right]}\end{array}$ & $\begin{array}{l}\rho \\
{\left[\mathrm{g} / \mathrm{cm}^{-}\right.} \\
\left.{ }_{3}\right]\end{array}$ & $\begin{array}{l}\mathbf{V} / \mathbf{Z} \\
{\left[\AA^{3}\right]}\end{array}$ & $\begin{array}{l}\mathbf{V}_{\text {solv }} \\
{\left[\AA^{3}\right]}\end{array}$ & $\begin{array}{l}\mathbf{V}_{\mathbf{m o l}} \\
{\left[\AA^{3}\right]}\end{array}$ \\
\hline$[4] \mathrm{C}-\mathrm{Hex}-\mathrm{Cbz}$ & -123 & $\begin{array}{l}a=9.4194, b=26.193, c= \\
12.3056, \beta=101.590 \\
\alpha=\gamma=90, V=2974.3(Z=2)\end{array}$ & 1.114 & 1487.1 & 0 & 1487.1 \\
\hline$[4] \mathrm{C}-H e x-C b z$ & 20 & $\begin{array}{l}a=9.419, b=26.27, c=12.341 \\
\beta=101.80 \\
\alpha=\gamma=90, V=2989.1(Z=2)\end{array}$ & 1.108 & 1494.5 & 0 & 1494.5 \\
\hline [4]C-Hex-Cbz & 180 & $\begin{array}{l}a=9.438, b=26.66, c=12.687 \\
\beta=103.43 \\
\alpha=\gamma=90, V=3105.0(Z=2)\end{array}$ & 1.067 & 1552.5 & 0 & 1552.5 \\
\hline$[4] \mathrm{C}-\mathrm{Bu}-\mathrm{Cbz}$ & -123 & $\begin{array}{l}a=17.0612, b=17.9109, c= \\
18.9041, \beta=90 \\
\alpha=\gamma=90, V=5776.7(Z=4)\end{array}$ & 1.124 & 1444.2 & 153 & 1291 \\
\hline [4]C-Et-Cbz & -123 & $\begin{array}{l}a=26.355, b=9.1895, c= \\
41.286, \beta=96.498 \\
\alpha=\gamma=90, V=9935(Z=8)\end{array}$ & 1.233 & 1241.9 & 145 & 1097 \\
\hline$[8] \mathrm{CPP}$ & 20 & $\begin{array}{l}a=13.327, b=8.060, c= \\
19.254, \beta=108.08 \\
\alpha=\gamma=90, V=1952.8(Z=2)\end{array}$ & 1.035 & 976.4 & 0 & 976.4 \\
\hline$[8] \mathrm{CPP}$ & -173 & $\begin{array}{l}a=12.9325, b=8.0103, c= \\
19.3676, \beta=105.363 \\
\alpha=\gamma=90, V=1934.66(Z=2)\end{array}$ & 1.045 & 967.63 & 0 & 967.63 \\
\hline
\end{tabular}


Table S 6 Structural parameters at temperature of measurement $T$.

\begin{tabular}{|c|c|c|c|c|c|c|c|}
\hline $\begin{array}{c}\text { Compound } \\
\mathrm{T}\left[{ }^{\circ} \mathrm{C}\right] \\
\text { Structure type }\end{array}$ & $\begin{array}{l}\text { 2D-sublattice parameters }\left[\AA,{ }^{\circ}, \AA^{2}\right] \\
\text { 3D-cell parameters }\left[\AA,{ }^{\circ}\right] \\
\text { Columnar lattice parameters }\left[\AA,{ }^{\circ}, \AA^{2}\right]\end{array}$ & $\begin{array}{l}\mathrm{h}_{\text {mol }}[\AA] \\
\mathrm{D}_{\text {row }}[\AA]\end{array}$ & $\begin{array}{l}\left.\mathrm{D}_{\mathrm{mol}}[\AA]\right] \\
\mathrm{d}_{\mathrm{lam}}[\AA]\end{array}$ & $\begin{array}{l}\mathrm{A}_{\mathrm{mol}}\left[\AA^{2}\right] \\
\mathrm{S}_{\mathrm{mol}}\left[\AA^{2}\right]\end{array}$ & $\begin{array}{c}\psi\left[^{\circ}\right] \\
\mathrm{h}_{\text {stack }}[\AA]\end{array}$ & $\begin{array}{l}\mathrm{s}_{\text {ring }}\left[\AA^{2}\right] \\
\sigma_{\text {ring }}\left[\AA^{2}\right]\end{array}$ & $\begin{array}{l}\left.\mathrm{D}_{\text {ring, cyl }}[\AA]\right] \\
\mathrm{D}_{\text {ring, cav }}[\AA]\end{array}$ \\
\hline $\begin{array}{c}{[4] \mathrm{C}-\mathrm{Hex}-\mathrm{Cbz}} \\
-123 \\
\text { LamCol }\end{array}$ & $\begin{array}{c}a_{2 \mathrm{D}}=26.193, b_{2 \mathrm{D}}=9.4194, \gamma_{2 \mathrm{D}}=90, A=246.72\left(Z_{2 \mathrm{D}}=2\right) \\
c^{\prime}=12.3056\left(N_{\mathrm{L}}=1\right), \alpha^{\prime}=101.590, \beta^{\prime}=90 \\
a_{\mathrm{col}}=26.193, b_{\mathrm{col}}=12.0547, \gamma_{\mathrm{col}}=90, A_{\mathrm{col}}=315.75\left(Z_{\mathrm{col}}=2\right)\end{array}$ & $\begin{array}{l}9.4194 \\
13.097\end{array}$ & $\begin{array}{l}12.055 \\
12.055\end{array}$ & $\begin{array}{l}123.36 \\
157.88\end{array}$ & $\begin{array}{l}51.4 \\
5.88\end{array}$ & $\begin{array}{c}95.7 \\
153.4\end{array}$ & $\begin{array}{l}13.31 \\
10.96\end{array}$ \\
\hline $\begin{array}{c}{[4] \mathrm{C}-\mathrm{Hex}-\mathrm{Cbz}} \\
20 \\
\text { LamCol }\end{array}$ & $\begin{array}{c}a_{2 \mathrm{D}}=26.27, b_{2 \mathrm{D}}=9.419, \gamma_{2 \mathrm{D}}=90, A=247.4\left(Z_{2 \mathrm{D}}=2\right) \\
c^{\prime}=12.341\left(N_{\mathrm{L}}=1\right), \alpha^{\prime}=101.80, \beta^{\prime}=90 \\
a_{\mathrm{col}}=26.27, b_{\mathrm{col}}=12.080, \gamma_{\mathrm{col}}=90, A_{\mathrm{col}}=317.3\left(Z_{\mathrm{col}}=2\right)\end{array}$ & $\begin{array}{c}9.419 \\
13.135\end{array}$ & $\begin{array}{l}12.08 \\
12.08\end{array}$ & $\begin{array}{c}123.7 \\
158.7\end{array}$ & - & - & - \\
\hline $\begin{array}{c}{[4] \mathrm{C}-\mathbf{H e x}-\mathbf{C b z}} \\
180 \\
\text { LamCol }\end{array}$ & $\begin{array}{c}a_{2 \mathrm{D}}=26.66, b_{2 \mathrm{D}}=9.438, \gamma_{2 \mathrm{D}}=90, A=251.6\left(Z_{2 \mathrm{D}}=2\right) \\
c^{\prime}=12.687\left(N_{\mathrm{L}}=1\right), \alpha^{\prime}=103.43, \beta^{\prime}=90 \\
a_{\mathrm{col}}=26.193, b_{\mathrm{col}}=12.340, \gamma_{\mathrm{col}}=90, A_{\mathrm{col}}=329.0\left(Z_{\mathrm{col}}=2\right)\end{array}$ & $\begin{array}{c}9.438 \\
13.330\end{array}$ & $\begin{array}{l}12.34 \\
12.34\end{array}$ & $\begin{array}{l}125.8 \\
164.5\end{array}$ & - & - & - \\
\hline $\begin{array}{l}{[4] \mathbf{C}-\mathbf{B u}-\mathbf{C b z}} \\
\bullet \mathrm{C}_{6} \mathrm{H}_{5} \mathrm{CH}_{3} \\
-123\end{array}$ & $\begin{array}{c}a_{2 \mathrm{D}}=18.9041, b_{2 \mathrm{D}}=17.9109, \gamma_{2 \mathrm{D}}=90, A=338.59\left(Z_{2 \mathrm{D}}=2\right) \\
c^{\prime}=17.0612\left(N_{\mathrm{L}}=2\right), \alpha^{\prime}=90, \beta^{\prime}=90\end{array}$ & $\begin{array}{l}8.9554 \\
9.4520\end{array}$ & $\begin{array}{c}8.5306 \\
-\end{array}$ & $\begin{array}{l}169.29 \\
161.26\end{array}$ & $\begin{array}{c}36.8 \\
-\end{array}$ & - & 10.92 \\
\hline $\begin{array}{c}{[4] \mathbf{C}-\mathbf{E t}-\mathbf{C b z}} \\
\bullet \mathrm{CHCl}_{3} \\
-123 \\
\text { LamCol } \\
\end{array}$ & $\begin{array}{c}a_{2 \mathrm{D}}=26.355, b_{2 \mathrm{D}}=9.1895, \gamma_{2 \mathrm{D}}=90, A=242.19\left(Z_{2 \mathrm{D}}=2\right) \\
c^{\prime}=41.286\left(N_{\mathrm{L}}=4\right), \alpha^{\prime}=90, \beta^{\prime}=96.498 \\
a_{\mathrm{col}}=41.286, b_{\mathrm{col}}=26.355, \gamma_{\mathrm{col}}=96.498, A_{\mathrm{col}}=1081.10\left(Z_{\mathrm{col}}=8\right)\end{array}$ & $\begin{array}{l}9.1895 \\
13.178\end{array}$ & $\begin{array}{l}10.255 \\
41.021\end{array}$ & $\begin{array}{l}121.10 \\
135.14\end{array}$ & $\begin{array}{l}50.8 \\
5.80\end{array}$ & $\begin{array}{c}98.1 \\
155.3\end{array}$ & $\begin{array}{l}13.39 \\
10.84\end{array}$ \\
\hline $\begin{array}{c}{[8] \mathbf{C P P}} \\
-173 \\
\mathrm{Col}\end{array}$ & $\begin{array}{c}a_{2 \mathrm{D}}=12.9325, b_{2 \mathrm{D}}=8.0103, \gamma_{2 \mathrm{D}}=90, A=103.59\left(Z_{2 \mathrm{D}}=1\right) \\
c^{\prime}=19.3676\left(N_{\mathrm{L}}=2\right), \alpha^{\prime}=90, \beta^{\prime}=105.363 \\
a_{\mathrm{col}}=19.3676, b_{\mathrm{col}}=12.9325, \gamma_{\mathrm{col}}=105.363, A_{\mathrm{col}}=241.52\left(Z_{\mathrm{col}}=2\right)\end{array}$ & $\begin{array}{c}8.0103 \\
12.9325\end{array}$ & $\begin{array}{c}9.338 \\
-\end{array}$ & $\begin{array}{l}103.59 \\
120.76\end{array}$ & $\begin{array}{l}43.8 \\
5.78\end{array}$ & $\begin{array}{c}103.6 \\
167\end{array}$ & $\begin{array}{l}13.9 \\
11.0\end{array}$ \\
\hline $\begin{array}{c}{[8] \mathbf{C P P}} \\
20 \\
\mathrm{Col}\end{array}$ & $\begin{array}{c}a_{2 \mathrm{D}}=13.237, b_{2 \mathrm{D}}=8.060, \gamma_{2 \mathrm{D}}=90, A=106.69\left(Z_{2 \mathrm{D}}=1\right) \\
c^{\prime}=19.254\left(N_{\mathrm{L}}=2\right), \alpha^{\prime}=90, \beta^{\prime}=108.08 \\
a_{\mathrm{col}}=19.254, b_{\mathrm{col}}=13.237, \gamma_{\mathrm{col}}=108.08, A_{\mathrm{col}}=242.3\left(Z_{\mathrm{col}}=2\right)\end{array}$ & $\begin{array}{c}8.060 \\
13.237\end{array}$ & $\begin{array}{c}9.15 \\
-\end{array}$ & $\begin{array}{l}106.7 \\
121.1\end{array}$ & - & - & - \\
\hline
\end{tabular}

LamCol: lamello-columnar structure; col: columnar structure; $a_{2 \mathrm{D}}, b_{2 \mathrm{D}}, \mathrm{g}_{2 \mathrm{D}}, A, Z_{2 \mathrm{D}}$ : sublattice parameters, area and number of molecules per sublattice in the molecular layer plane; $c^{\prime}, N_{\mathrm{L}}, \mathrm{a}^{\prime}, \mathrm{b}^{\prime}$ : cell parameters after referential change ( $a^{\prime}=a_{2 \mathrm{D}}$ : direction of row juxtaposition; $b^{\prime}=b_{2 \mathrm{D}}$ : direction of rows; $c^{\prime}$ : direction of layer normal; $N_{\mathrm{L}}:$ number of layers per cell); $a_{\mathrm{col}}, b_{\mathrm{col}}, \gamma_{\mathrm{col}}, A_{\mathrm{col}}, Z_{\mathrm{col}}$; columnar lattice parameters, lattice area and number of columns per lattice ([4]C-Hex-Cbz: $\left.b_{\mathrm{col}}=c^{\prime} \times \cos \left(\alpha^{\prime}\right)\right)$; $\mathrm{h}_{\mathrm{mol}}$ : molecular spacing along rows/columns; $\mathrm{D}_{\mathrm{row}}$ : row/columns spacing in the layer plane; $\mathrm{D}_{\mathrm{mol}}$ : thickness of molecular layers; $\mathrm{d}_{\mathrm{lam}}=\mathrm{N}_{\mathrm{L}} \times \mathrm{D}_{\mathrm{mol}}$ : lamellar periodicity; $\mathrm{A}_{\mathrm{mol}}=\mathrm{V}_{\mathrm{mol}} / \mathrm{D}_{\mathrm{mol}}=A / Z_{2 \mathrm{D}}$ : molecular area in the layer plane; $\mathrm{S}_{\mathrm{mol}}=\mathrm{V}_{\mathrm{mol}} / \mathrm{h}_{\mathrm{mol}}=A_{\mathrm{col}} / Z_{\mathrm{col}}$ : molecular section in the columnar/piling plane; $\psi$ : tilt angle of rings; $\mathrm{h}_{\text {stack }}=\mathrm{h}_{\text {mol }} \times \cos (\psi)$ : stacking distance of rings; $\mathrm{S}_{\text {ring }}=\mathrm{V}_{\text {ring }} / \mathrm{h}_{\text {mol }}$ : cross-sectional area of stacked rings in the columnar plane; $\mathrm{s}_{\text {ring }}=\mathrm{V}_{\text {ring }} / \mathrm{h}_{\text {stack }}$ : crosssectional area of rings; $D_{\text {ring, cyl }}=\sqrt{ }\left((2 / \sqrt{ } 3) \times S_{\text {ring }}\right)$ : diameter of equivalent cylinder of cross-sectional area $\mathrm{S}_{\text {ring }}$; $D_{\text {ring,cav }}$ : average ring cavity diameter from C-C distances (the shape of the [8]CPP ring is approximately elliptical: $D_{1}=11.66 \AA, D_{2}=10.36 \AA ; D_{\text {ring, cav }}=\sqrt{ }\left(D_{1} \times D_{2}\right)=10.99$; flattening: $\left.e=1-D_{2} / D_{1}=0.1114=1 / 8.97\right)$ 
Packing diagrams

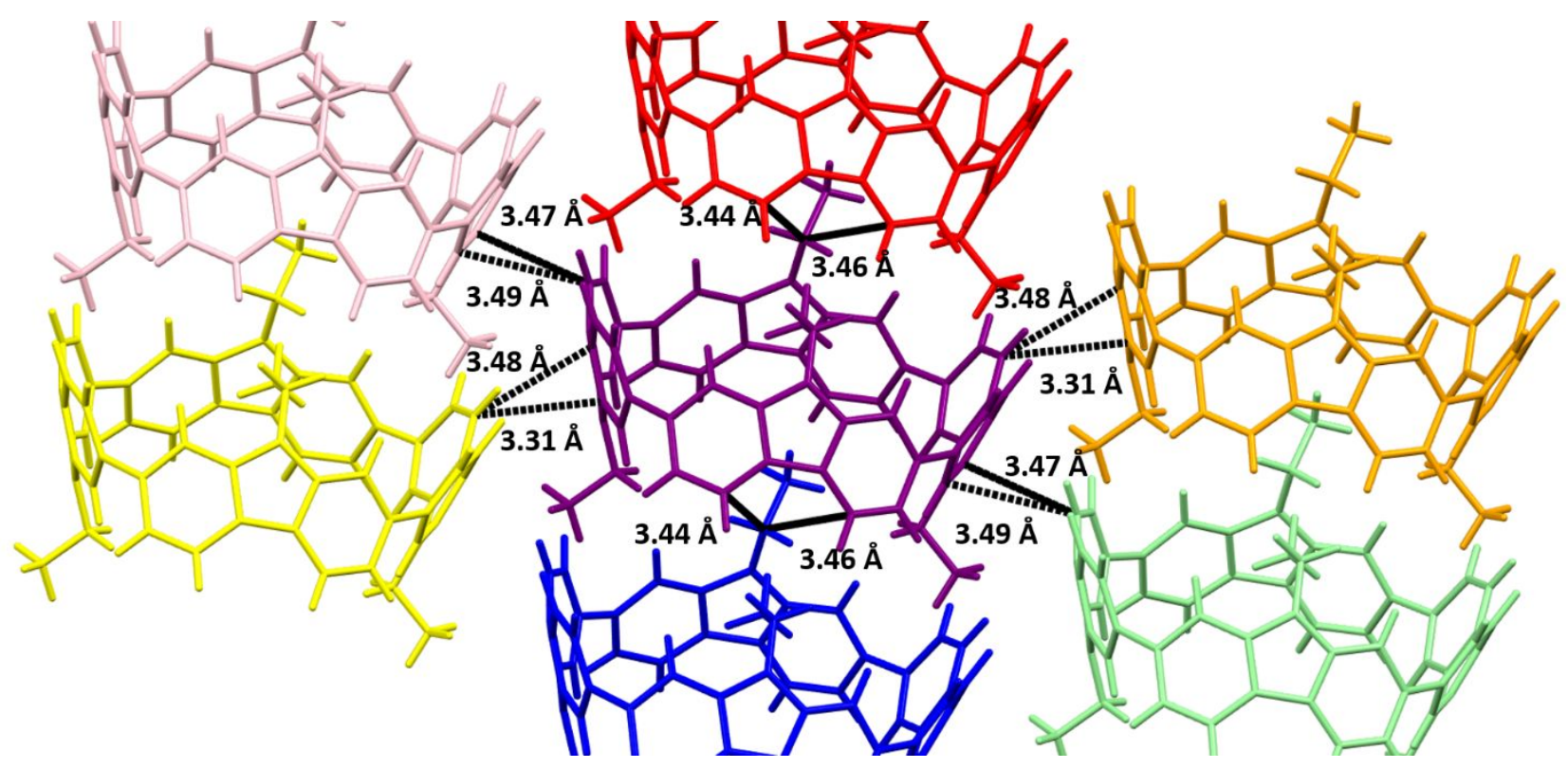

Figure S 16 Short $C$-C distances in $[\mathbf{4 J C}-\mathbf{E t}-\mathbf{C b z}$ single crystal packing diagram

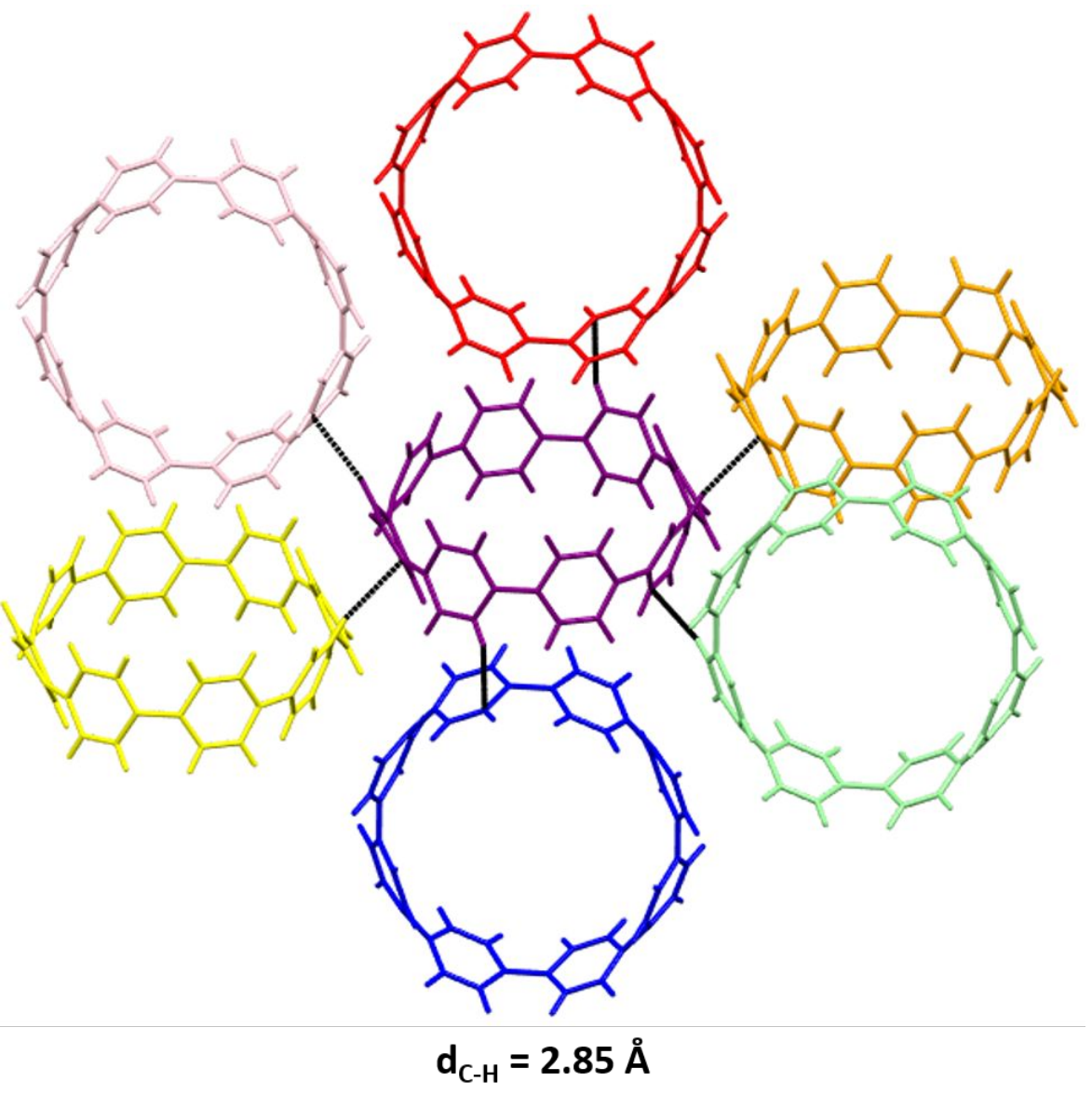

Figure $S 17$ Short $C$-H distances in [8/CPP single crystal packing diagram 


\section{Electrochemical studies}
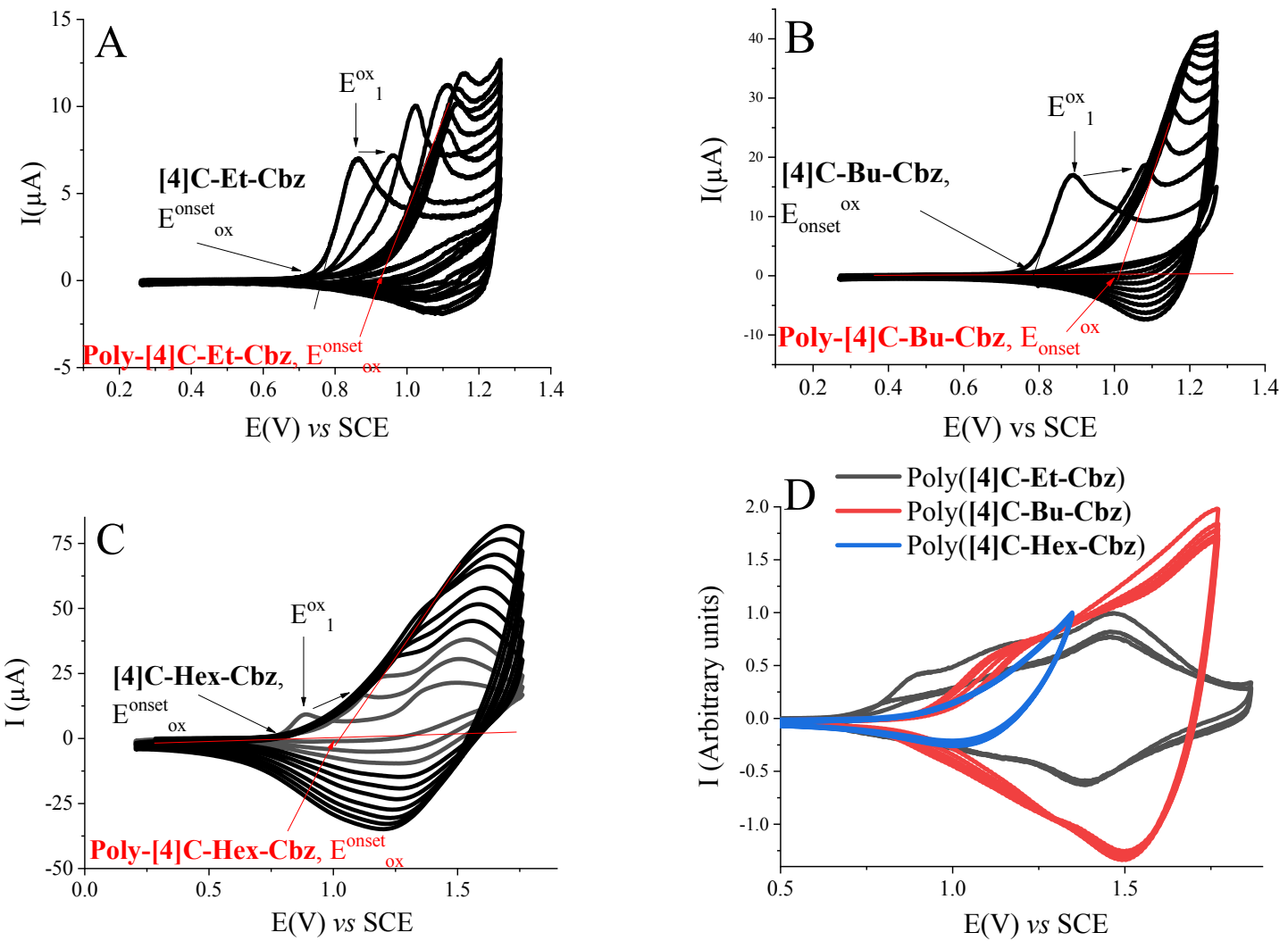

Figure $S 18$ Cyclic voltammograms recorded in $D C M+B u_{4} N P F_{6} 0.2 M$ in presence of [4]C-Et-Cbz (A), [4]C-Bu-Cbz, (B) and [4]C-Hex-Cbz,(C). Ten recurrent cycles. Platinum Working electrode, $100 \mathrm{mV} / \mathrm{s}$. Cyclic voltammograms recorded in $\mathrm{DCM}+\mathrm{Bu}_{4} \mathrm{NPF} \mathrm{F}_{6} 0.2 \mathrm{M}$ in absence of the cyclocarbazoles [4]C-alkyl-Cbz (D). Poly([4]C-alkyl-Cbz) modified platinum electrode. $100 \mathrm{mV} / \mathrm{s}$.

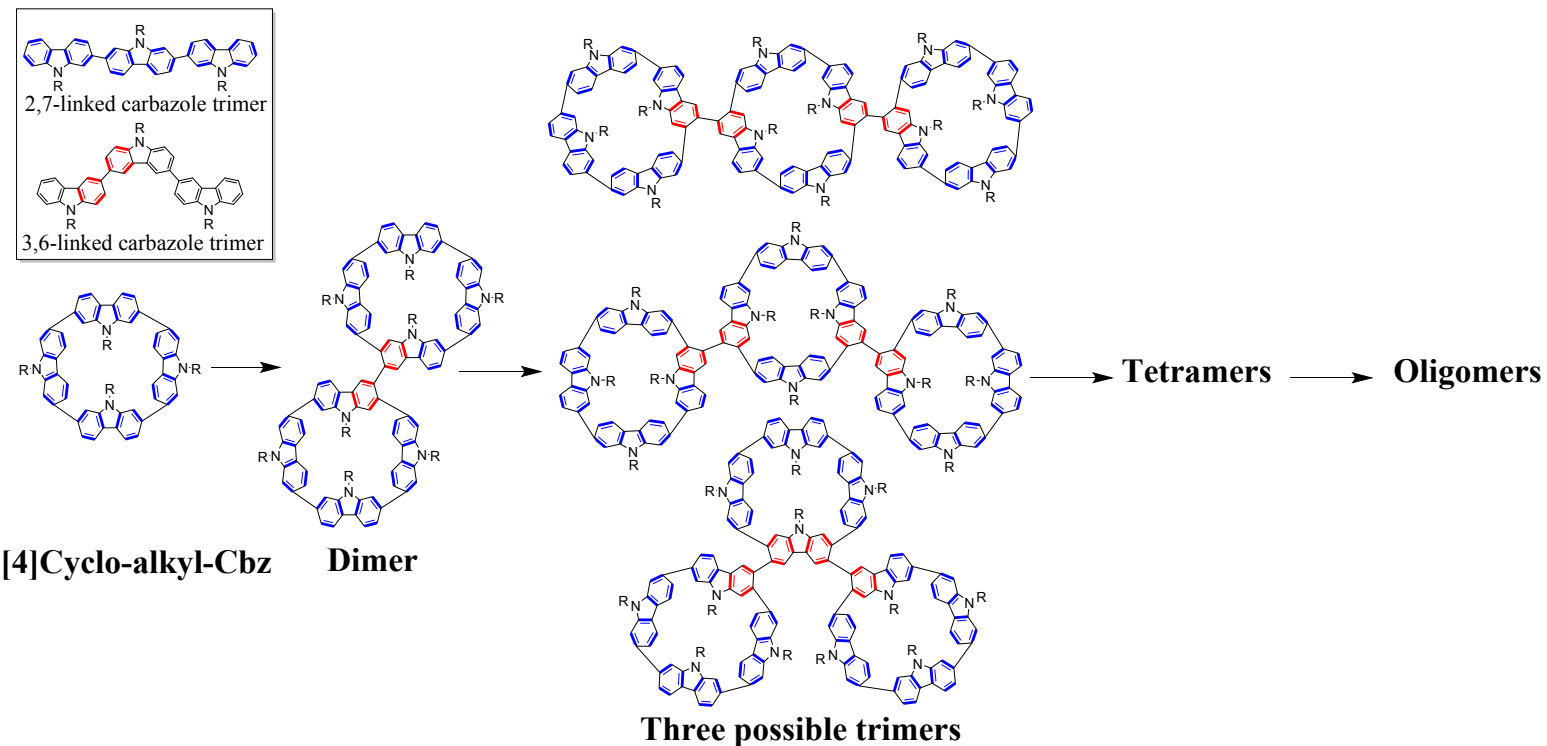

Figure S 19 Proposed carbon-carbon coupling leading to electrodeposition process along recurrent cycles. 


\section{Photophysical properties}

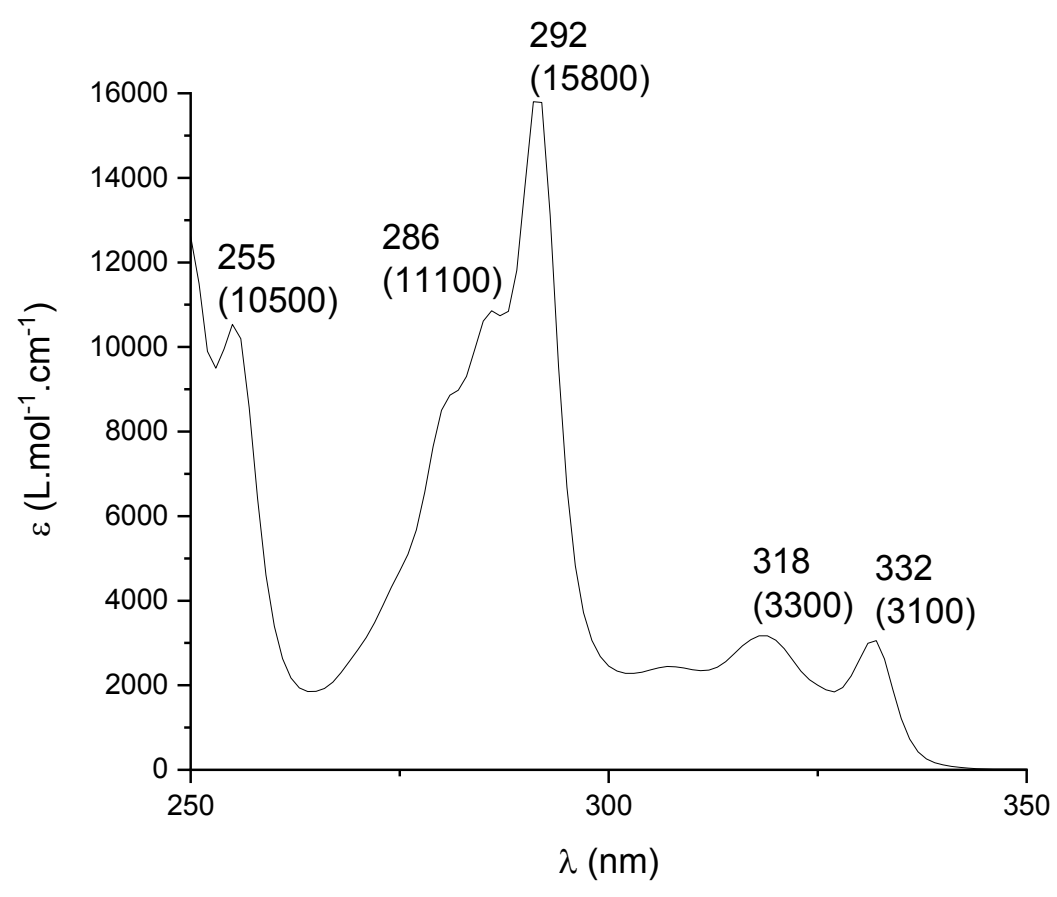

Figure S 20. Absorption spectra of $\mathbf{C b z}$ in cyclohexane

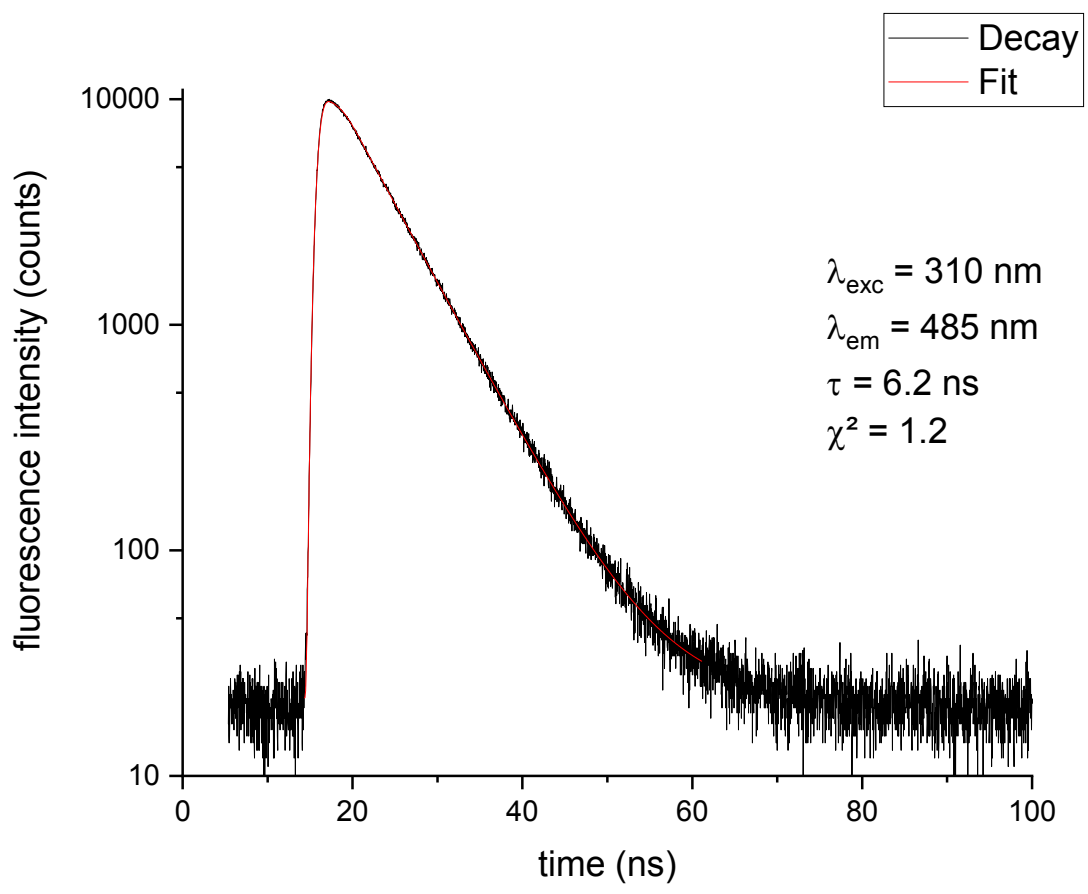

Figure S 21 Fluorescence decay of [4]C-Et-Cbz in cyclohexane 


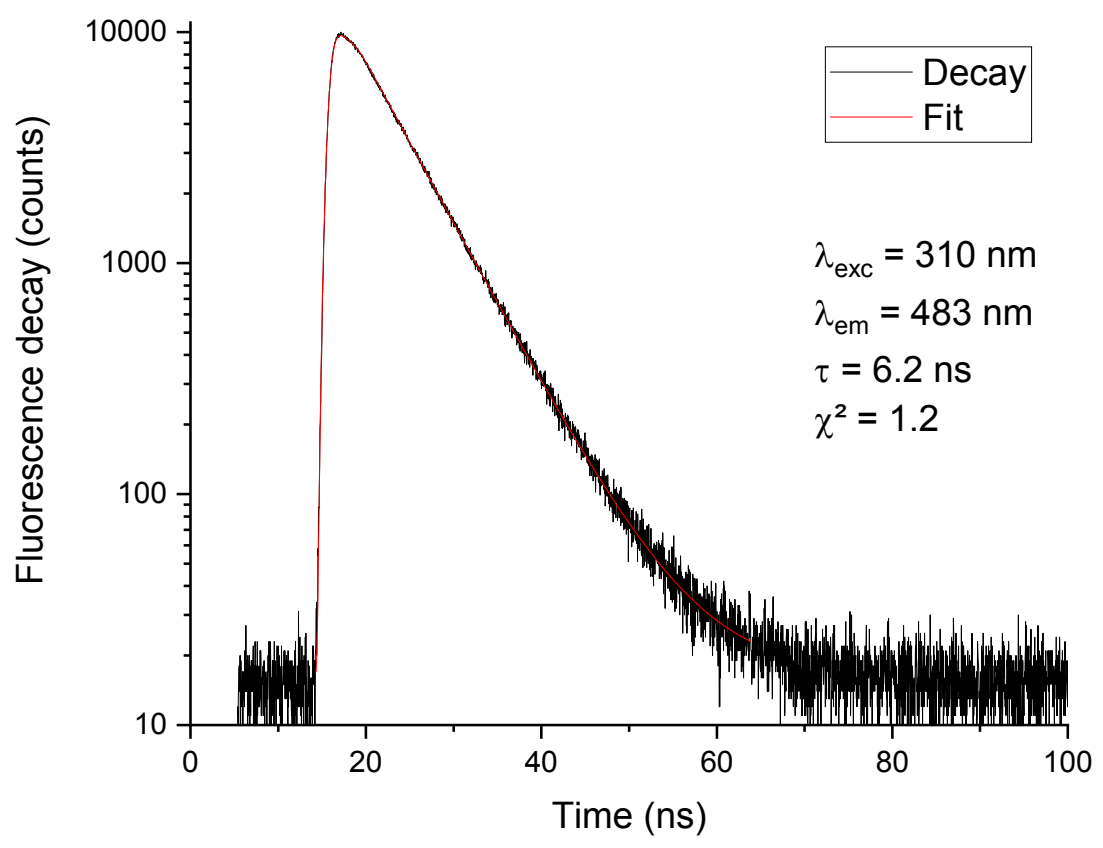

Figure S 22 Fluorescence decay of [4]C-Bu-Cbz in cyclohexane

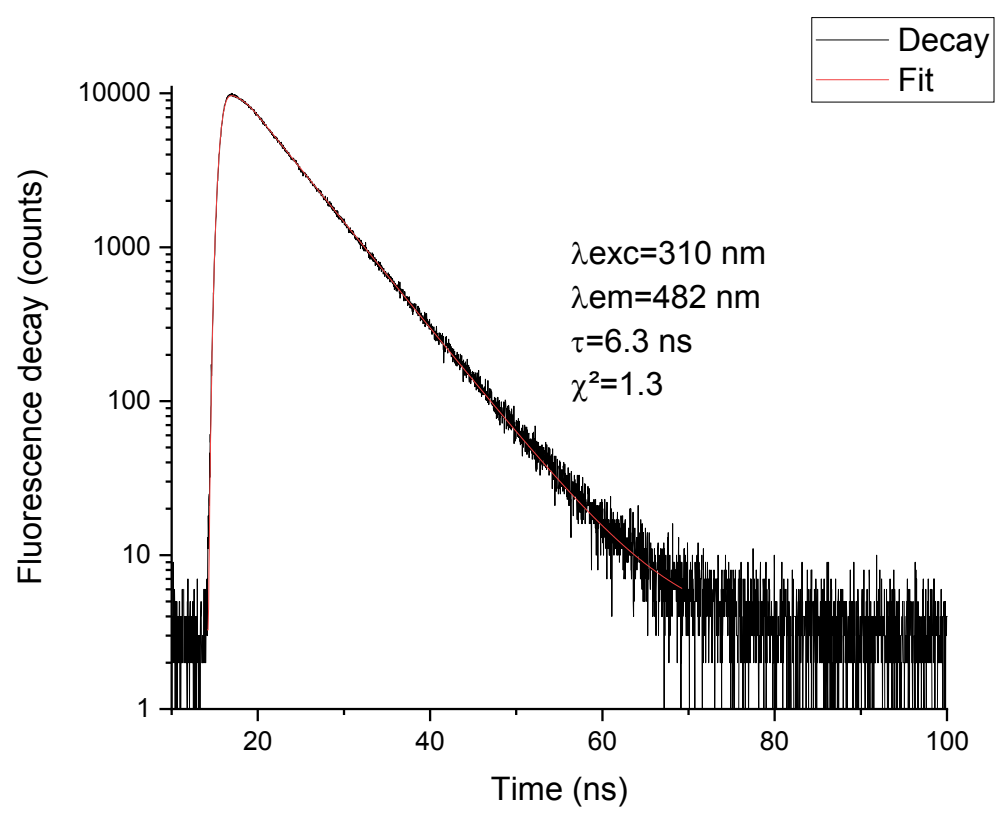

Figure S 23 Fluorescence decay of [4]C-Hex-Cbz in cyclohexane 


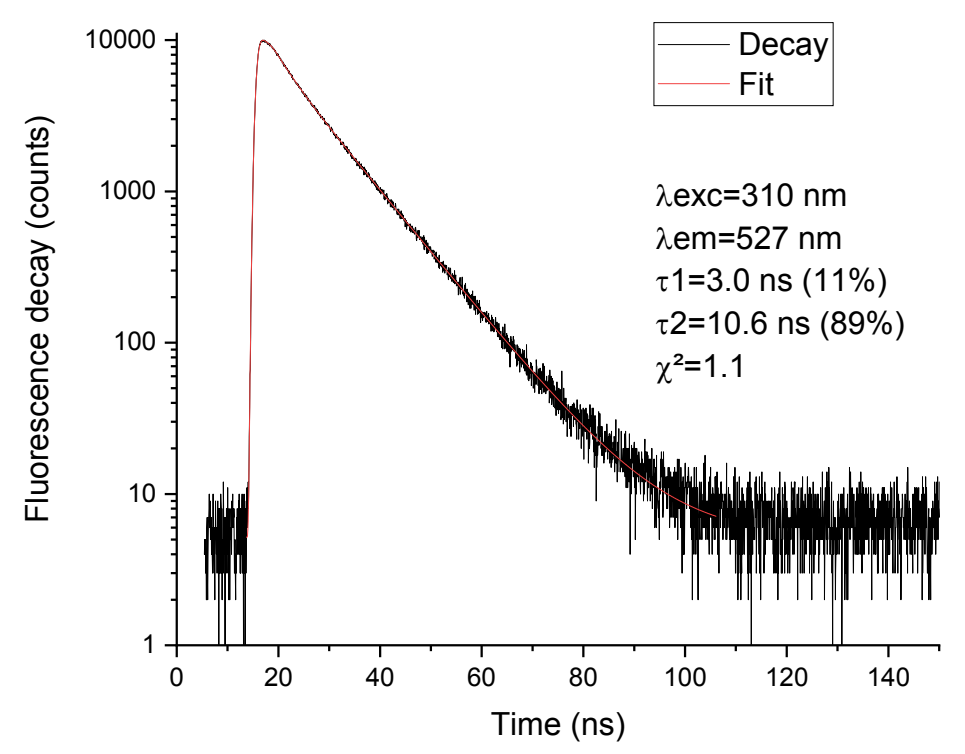

Figure S 24 Fluorescence decay of [8/CPP in cyclohexane

\section{Organic field effect transistor and space-charged-limited current diode} measurements

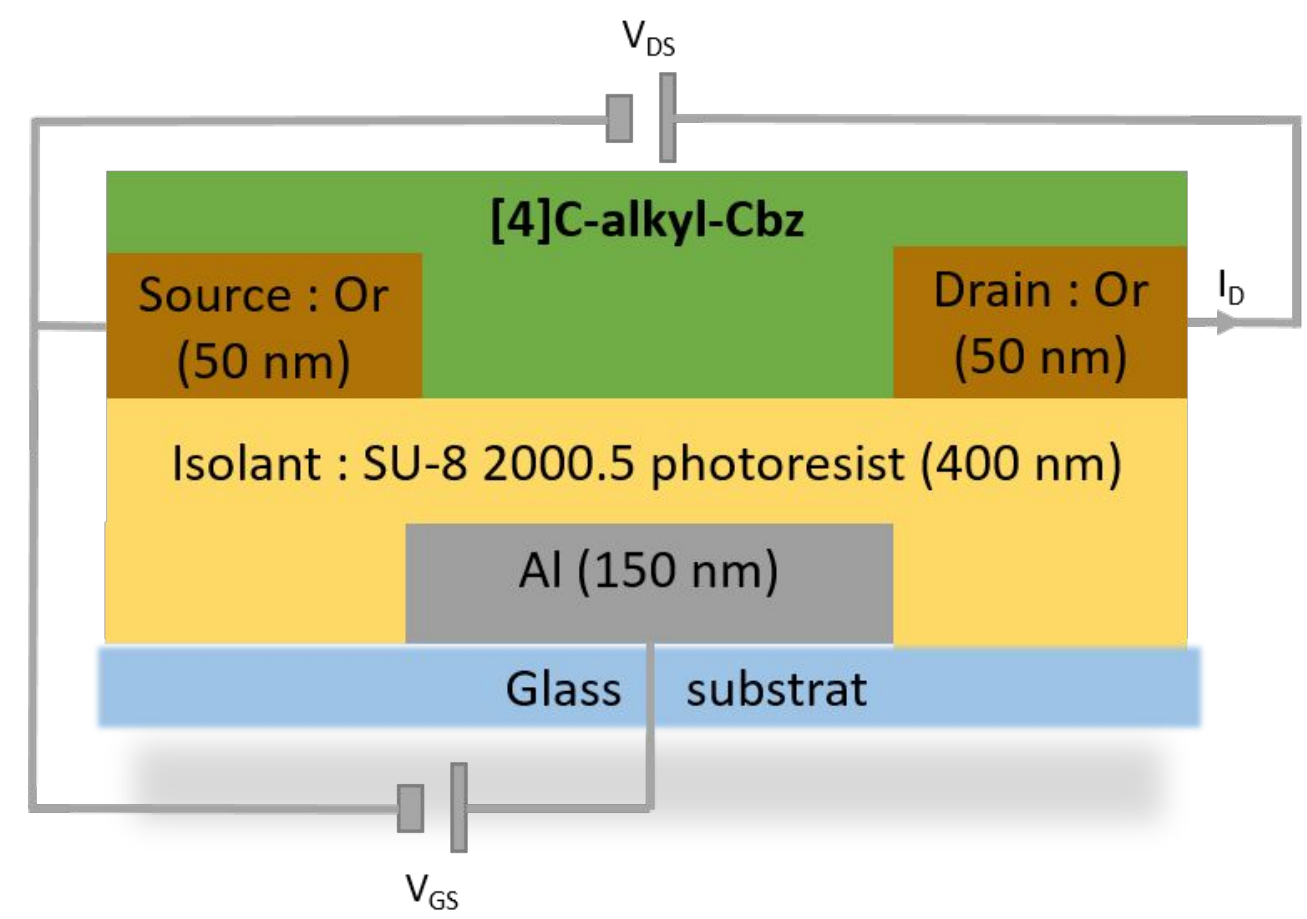

Figure S 25 Structure of the bottom-gate bottom-contact p-type channel OFETs on glass substrat

For the three nanorings the carrier mobility is extracted using the Mott-Gurney formalism in the area where $m=2$ where the traps are no longer involved in the electrical conduction through the semiconducting layer. 


$$
\begin{aligned}
& J_{S C L C}=\frac{9}{8} \varepsilon_{0} \varepsilon_{r} \Theta \mu \frac{V^{2}}{L^{3}} \\
& \Theta=\frac{n_{f}}{n_{f}+n_{t}} \\
& m=\frac{\partial \log (J)}{\partial \log (V)}
\end{aligned}
$$

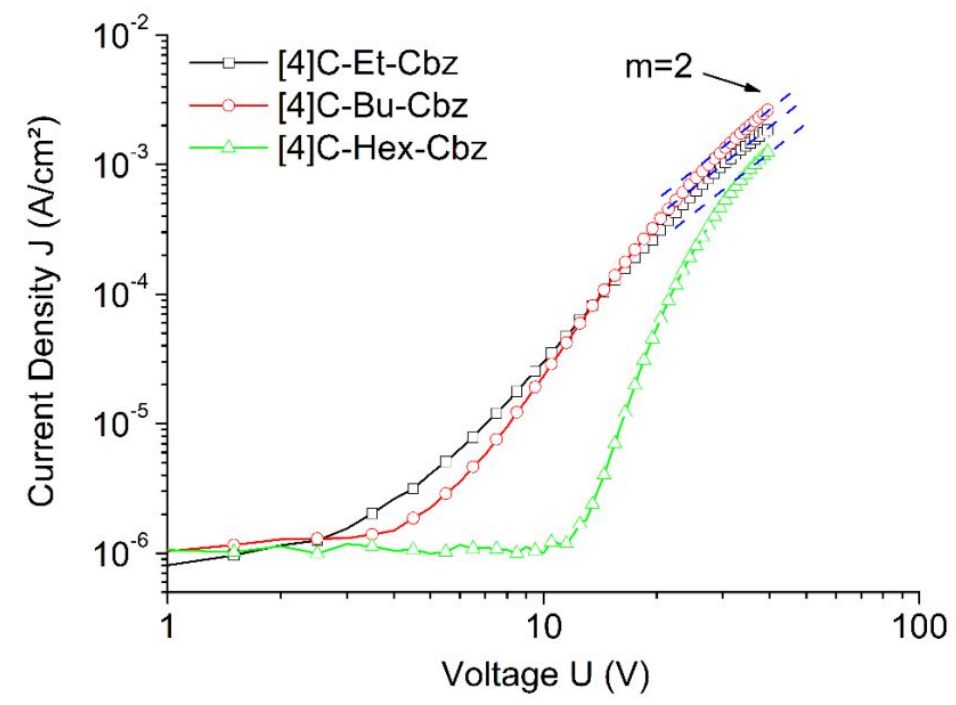

Figure $S 26$ I-V characteristics of Au/OSC/Au structures for [4]C-Et-Cbz, [4]C-Bu-Cbz and [4]C$\mathrm{Hex}-\mathrm{Cbz}$.

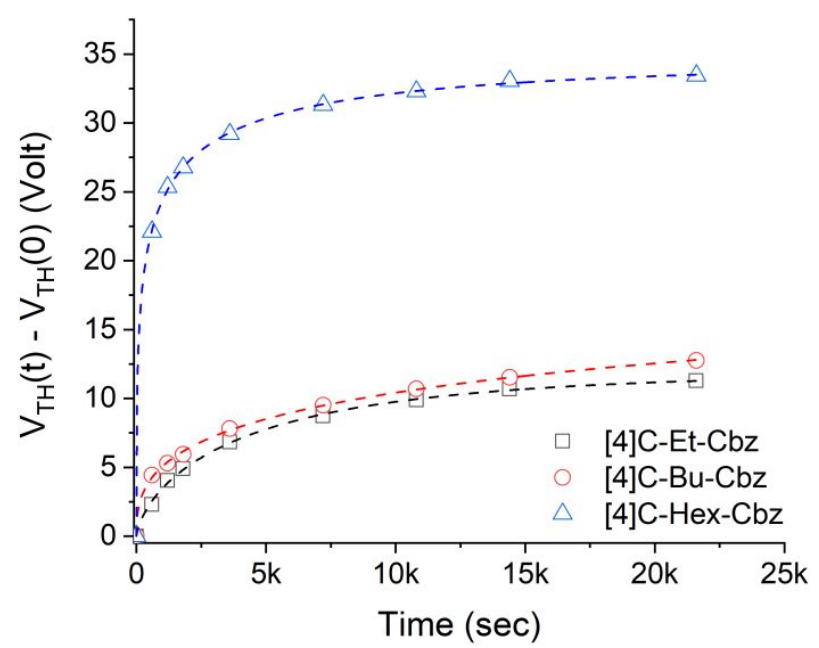

Figure $S 27$ Evolution of $\triangle V_{T H}$ with the stress time for [4]C-Et-Cbz, [4]C-Bu-Cbz and [4]C-Hex-Cbz based OFETs under $V_{G S s t r e s s}-V_{T H}=40 \mathrm{~V}$ and $V_{D S}=10 \mathrm{~V}$. The dashed lines are the fit with stretched exponential equation (4).

$$
V_{T H}(t)-V_{T H}(0)=V_{T H}(\infty)\left\{1-\exp \left[-\left({ }^{t} / t_{0}\right)^{\beta}\right]\right\}
$$



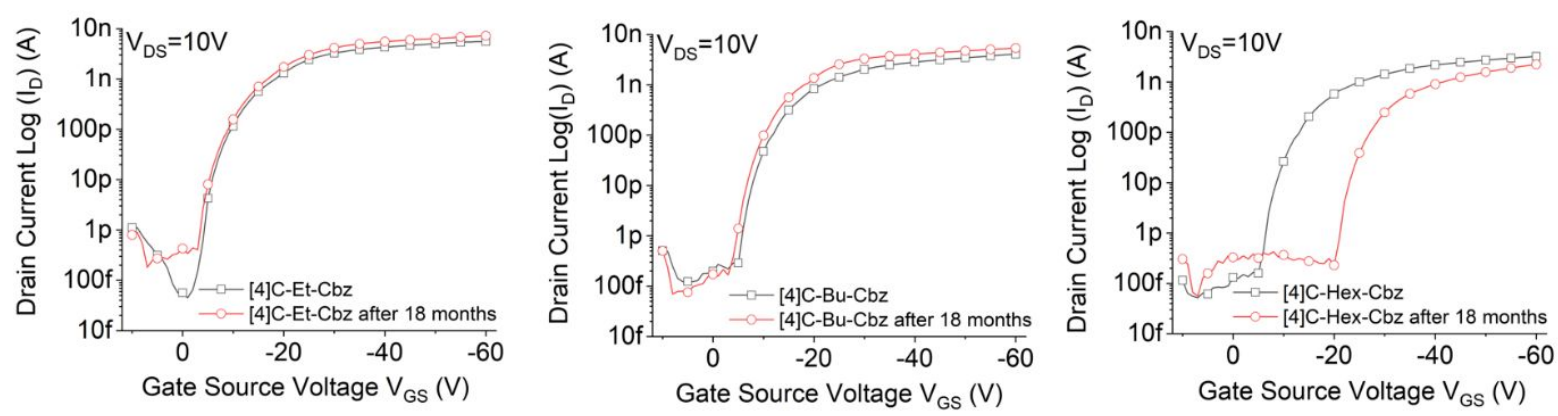

Figure $S 28$ Transfer characteristics in linear regime before and after 18 months of [4]C-Et-Cbz (left), [4]C-Bu-Cbz (middle) and [4]C-Hex-Cbz (right).

Table $S 7$ Stretched Exponential fit parameters. $V_{T H}(\infty)$ is the maximum threshold voltage shift after infinite stress time, $\beta$ is the stretched-exponential exponent and $t_{0}$ represents the characteristic trapping time of carriers.

\begin{tabular}{|c|c|c|c|c|}
\hline & $\mathrm{V}_{\mathrm{TH}}(\infty)(\mathrm{V})$ & $\beta$ & $\mathrm{t}_{0(\mathrm{~s})}$ & $\mathrm{R}^{2}$ \\
\hline$[4] \mathbf{C}-\mathbf{E t}-\mathbf{C b z}$ & 11.9 & 0.697 & 4623.4 & 0.9988 \\
\hline$[4] \mathbf{C}-\mathbf{B u}-\mathbf{C b z}$, & 23.3 & 0.384 & 39045.2 & 0.9992 \\
\hline$[4] \mathbf{C}-H e x-C b z$ & 34.7 & 0.335 & 558.9 & 0.9999 \\
\hline
\end{tabular}

Table $S 8$ Roughness parameters extracted from AFM images $\left(500 \times 500 \mathrm{~nm}^{2}\right)$

\begin{tabular}{|c|c|c|c|}
\hline & $\begin{array}{c}\mathrm{R}_{\mathrm{a}} \\
\text { (Arithmetic Average } \\
\text { Roughness) } \\
{[\mathrm{nm}]}\end{array}$ & $\begin{array}{c}\mathrm{R}_{\mathrm{q}} \\
\text { (RMS Roughness) }\end{array}$ & $\begin{array}{c}\mathrm{R}_{\max } \\
\text { (Maximum } \\
\text { Roughness depth) } \\
{[\mathrm{nm}]}\end{array}$ \\
\hline$[\mathbf{4}] \mathbf{C}-\mathbf{E t}-\mathbf{C b z}$ & 0.658 & {$[\mathrm{~nm}]$} & 4.81 \\
\hline$[4] \mathbf{C}-\mathbf{B u}-\mathbf{C b z}$, & 0.332 & 0.837 & 3.15 \\
\hline$[\mathbf{4}] \mathbf{C}-\mathbf{H e x}-\mathbf{C b z}$ & 1.18 & 0.421 & 9.46 \\
\hline$[\mathbf{8}] \mathbf{C P P}$ & 2.79 & 1.47 & 29.8 \\
\hline
\end{tabular}




\section{Molecular modelling}

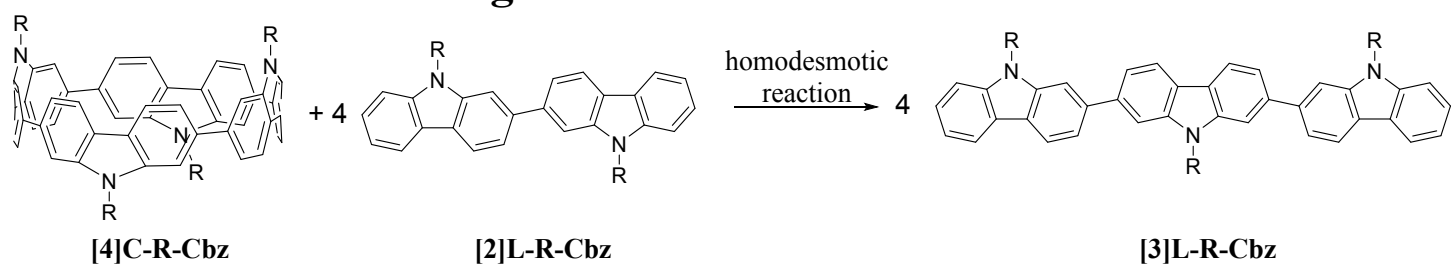

$\Delta \mathrm{H}=\mathrm{H}[\mathbf{4}] \mathbf{C}-\mathbf{R}-\mathbf{C b z}-4 * \mathrm{H}[3] \mathbf{L}-\mathbf{R}-\mathbf{C b z}+4 * \mathrm{H}[2] \mathbf{L}-\mathbf{R}-\mathbf{C b z}$

Figure S 29 Strain energy $(\Delta H)$ calculation (b3lyp/6-3lg $(d)$ )

Table $S 9$ Strain energies of $[4] \boldsymbol{C}-\mathbf{E} \boldsymbol{t}-\mathbf{C b z},[4] \boldsymbol{C}-\mathbf{B u}-\mathbf{C b z}$ and $[4] \boldsymbol{C}-\mathbf{H e x}-\mathbf{C b z}$. Energies are given in kcal.mol $^{-1}$.

\begin{tabular}{|c|c|c|c|c|}
\hline & -747070.99 & -1120239.18 & 73 \\
\hline$[4] \mathbf{C}-\mathbf{E t - C b z}$ & -1492599.30 & -845674.36 & -1268144.22 & 72 \\
\hline$[4] \mathbf{C}-B u-C b z$ & -1689807.53 & -944273.71 & 1416044.15 & 73 \\
\hline $\begin{array}{c}{[4] \mathbf{C}-H e x-} \\
\mathbf{C b z}\end{array}$ & -1887008.40 & & \\
\hline
\end{tabular}

$[8] C P P$

[4]C-diEt-F
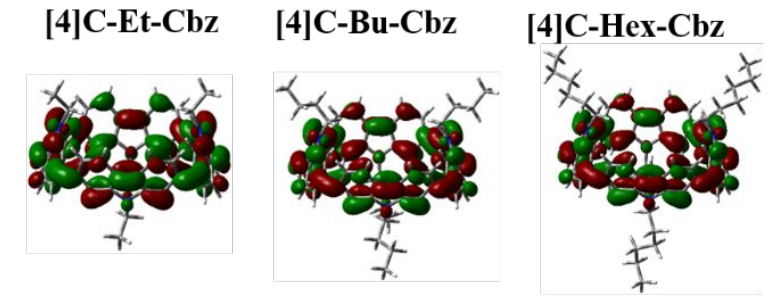

Et-Cbz

LUMO
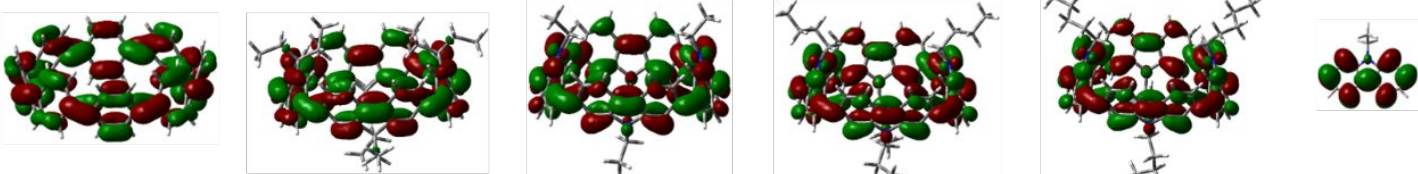

HOMO
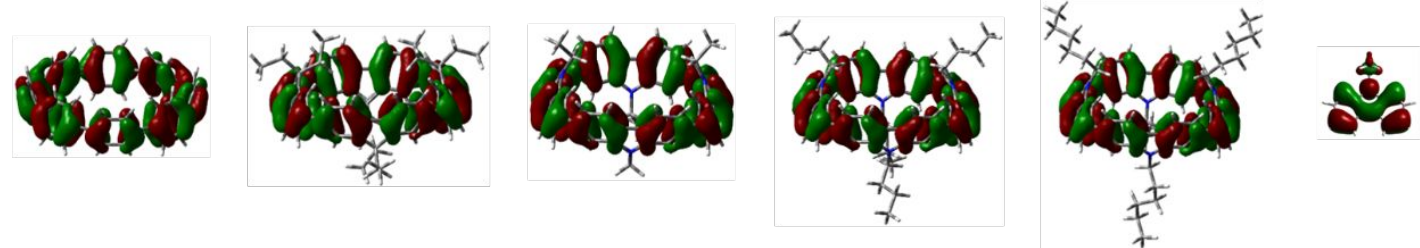

Figure $S 30$ Representation of the frontier orbitals of [8]CPP, [4]C-diEt-F, [4]C-Et-Cbz, [4]C-Bu-Cbz, [4]C-Hex-Cbz and $\boldsymbol{C b z}$ obtained by DFT, B3LYP/6-311+G(d,p), shown with an isovalue of $0.02\left[\mathrm{e} \mathrm{bohr^{-3 }}\right]^{1 / 2}$. 


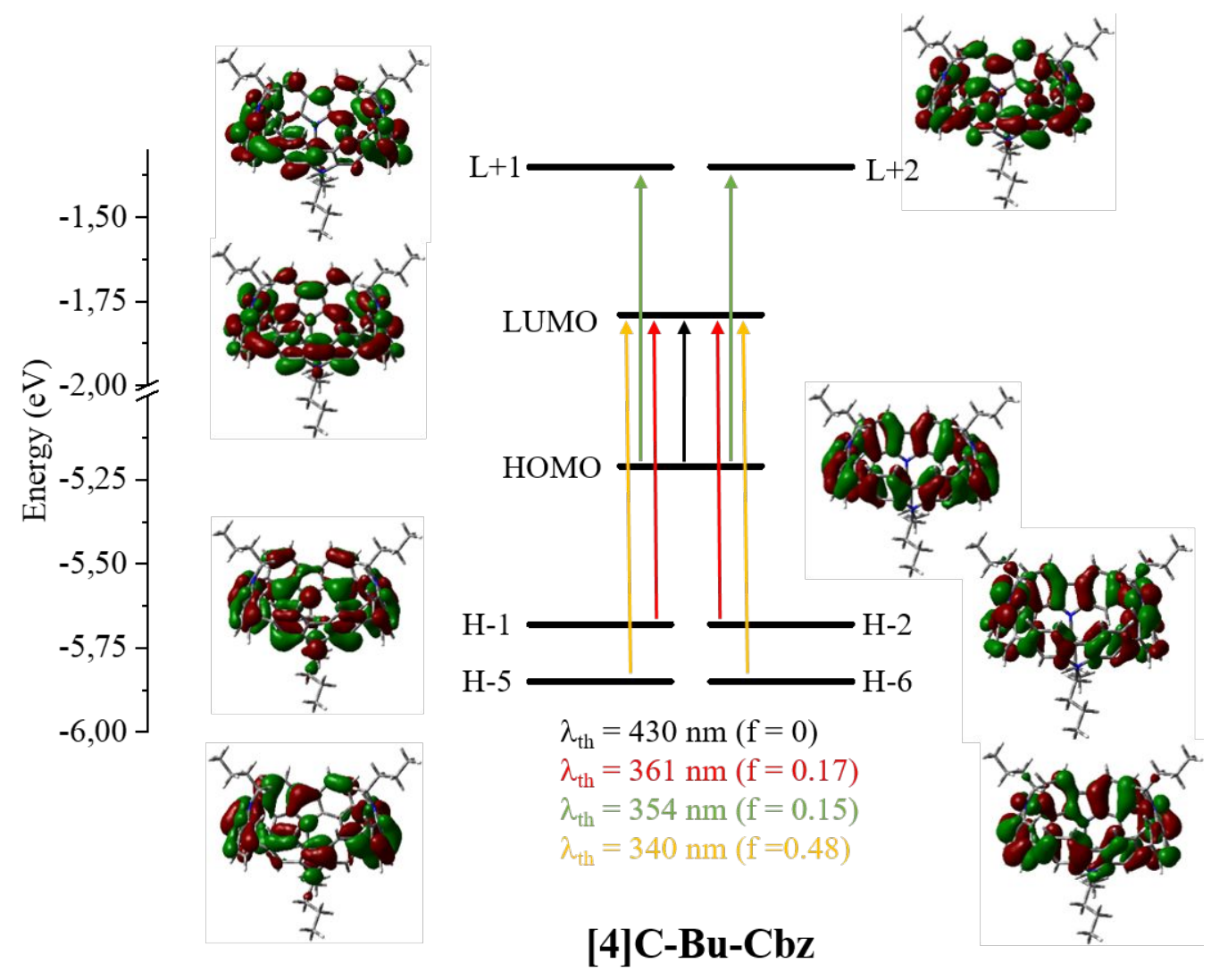

Figure S 31 Representation of the energy levels and the main molecular orbitals involved in the electronic transitions of [4]-Bu-Cbz obtained by TD-DFT, B3LYP/6-311 + G(d,p), shown with an isovalue of $0.02[\mathrm{e} \text { bohr-3 }]^{1 / 2}$. For clarity purposes, only the major contribution of each transition is shown. 


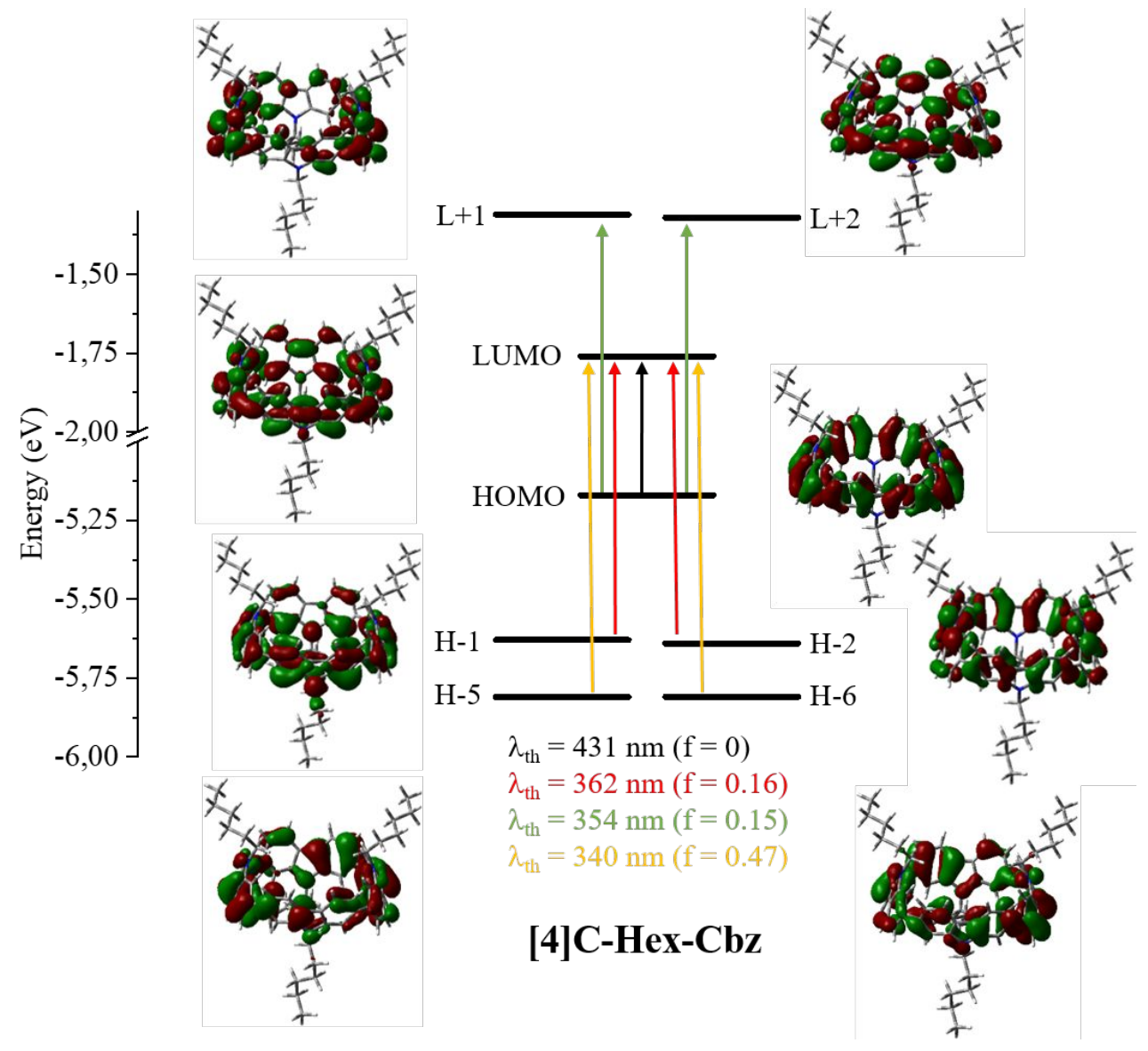

Figure S 32 Representation of the energy levels and the main molecular orbitals involved in the electronic transitions of [4]-Hex-Cbz obtained by TD-DFT, B3LYP/6-311 + G(d,p), shown with an isovalue of $0.02[\mathrm{e} \text { bohr-3 }]^{1 / 2}$. For clarity purposes, only the major contribution of each transition is shown.

Table S 10 TD-DFT results of [4]C-Et-Cbz (b3lyp/6-311+g(d,p).

\begin{tabular}{|c|c|c|c|}
\hline$\lambda(\mathrm{nm})$ & $\begin{array}{l}\text { Oscillator } \\
\text { strength }\end{array}$ & Major contributions & Minor contributionss \\
\hline 430 & 0 & $\mathrm{HOMO} \rightarrow \mathrm{LUMO}(97 \%)$ & \\
\hline 361 & 0.17 & $\begin{array}{l}\mathrm{H}-1 \rightarrow \mathrm{LUMO}(60 \%) \\
\mathrm{HOMO} \rightarrow \mathrm{L}+1(12 \%)\end{array}$ & $\begin{array}{c}\mathrm{H}-6 \rightarrow \operatorname{LUMO}(7 \%), \mathrm{H}-5 \rightarrow \mathrm{LUMO}(2 \%), \\
\mathrm{H}-3 \rightarrow \mathrm{L}+2(3 \%), \mathrm{HOMO} \rightarrow \mathrm{L}+2(7 \%)\end{array}$ \\
\hline 361 & 0.16 & $\begin{array}{l}\mathrm{H}-2 \rightarrow \mathrm{LUMO}(59 \%) \\
\mathrm{HOMO} \rightarrow \mathrm{L}+2(11 \%)\end{array}$ & $\begin{array}{c}\mathrm{H}-6 \rightarrow \mathrm{LUMO}(2 \%), \mathrm{H}-5 \rightarrow \mathrm{LUMO}(7 \%), \\
\mathrm{H}-3 \rightarrow \mathrm{L}+1(3 \%), \mathrm{H}-3 \rightarrow \mathrm{L}+2(2 \%), \\
\mathrm{HOMO} \rightarrow \mathrm{L}+1(8 \%)\end{array}$ \\
\hline 358 & 0.001 & H-3 $\rightarrow$ LUMO (88\%) & $\mathrm{H}-6 \rightarrow \mathrm{L}+2(3 \%), \mathrm{H}-5 \rightarrow \mathrm{L}+1(3 \%)$ \\
\hline 356 & 0.06 & $\mathrm{H}-4 \rightarrow$ LUMO $(84 \%)$ & $\begin{array}{c}\mathrm{H}-2 \rightarrow \mathrm{L}+2(3 \%), \mathrm{H}-1 \rightarrow \mathrm{L}+1(4 \%), \\
\mathrm{HOMO} \rightarrow \mathrm{L}+5(3 \%)\end{array}$ \\
\hline 354 & 0.13 & $\begin{array}{l}\mathrm{H}-2 \rightarrow \mathrm{LUMO}(12 \%) \\
\mathrm{H}-1 \rightarrow \mathrm{LUMO}(13 \%) \\
\mathrm{HOMO} \rightarrow \mathrm{L}+1(72 \%)\end{array}$ & \\
\hline
\end{tabular}




\begin{tabular}{|c|c|c|c|}
\hline \multirow{3}{*}{354} & \multirow{3}{*}{0.14} & \multicolumn{2}{|l|}{$\mathrm{H}-2 \rightarrow$ LUMO $(12 \%)$} \\
\hline & & \multicolumn{2}{|l|}{ H-1 $\rightarrow$ LUMO (11\%) } \\
\hline & & \multicolumn{2}{|l|}{$\mathrm{HOMO} \rightarrow \mathrm{L}+2(74 \%)$} \\
\hline \multirow{2}{*}{340} & \multirow{2}{*}{0.48} & \multirow{2}{*}{$\mathrm{H}-5 \rightarrow \mathrm{LUMO}(78 \%)$} & $\mathrm{H}-4 \rightarrow \mathrm{L}+2(4 \%), \mathrm{H}-2 \rightarrow \mathrm{LUMO}(7 \%)$ \\
\hline & & & $\mathrm{H}-1 \rightarrow \mathrm{LUMO}(3 \%), \mathrm{HOMO} \rightarrow \mathrm{L}+2(2 \%)$ \\
\hline \multirow{2}{*}{340} & \multirow{2}{*}{0.47} & \multirow{2}{*}{$\mathrm{H}-6 \rightarrow \mathrm{LUMO}(78 \%)$} & $\mathrm{H}-4 \rightarrow \mathrm{L}+1(4 \%), \mathrm{H}-2 \rightarrow \mathrm{LUMO}(3 \%)$ \\
\hline & & & $\mathrm{H}-1 \rightarrow \mathrm{LUMO}(6 \%), \mathrm{HOMO} \rightarrow \mathrm{L}+1(2 \%)$ \\
\hline \multirow{3}{*}{325} & \multirow{3}{*}{0.0001} & $\mathrm{H}-2 \rightarrow \mathrm{L}+1(16 \%)$ & \\
\hline & & $\mathrm{H}-1 \rightarrow \mathrm{L}+2(16 \%)$ & $\mathrm{H}-2 \rightarrow \mathrm{L}+2(2 \%), \mathrm{H}-1 \rightarrow \mathrm{L}+1(2 \%)$ \\
\hline & & $\mathrm{HOMO} \rightarrow \mathrm{L}+3(55 \%)$ & \\
\hline \multirow{3}{*}{321} & \multirow{3}{*}{0.006} & $\mathrm{H}-4 \rightarrow \mathrm{LUMO}(12 \%)$ & \multirow{3}{*}{$\begin{array}{c}\mathrm{H}-3 \rightarrow \mathrm{L}+3(5 \%), \mathrm{H}-2 \rightarrow \mathrm{L}+1(6 \%) \\
\mathrm{H}-1 \rightarrow \mathrm{L}+2(3 \%)\end{array}$} \\
\hline & & $\mathrm{H}-2 \rightarrow \mathrm{L}+2(28 \%)$ & \\
\hline & & $\mathrm{H}-1 \rightarrow \mathrm{L}+1(41 \%)$ & \\
\hline \multirow{4}{*}{319} & \multirow{4}{*}{0} & $\mathrm{H}-2 \rightarrow \mathrm{L}+1(17 \%)$ & \\
\hline & & $\mathrm{H}-2 \rightarrow \mathrm{L}+2(36 \%)$ & \\
\hline & & $\mathrm{H}-1 \rightarrow \mathrm{L}+1(24 \%)$ & \\
\hline & & $\mathrm{H}-1 \rightarrow \mathrm{L}+2(19 \%)$ & \\
\hline \multirow{4}{*}{317} & \multirow{4}{*}{0.0001} & $\mathrm{H}-2 \rightarrow \mathrm{L}+1(38 \%)$ & \multirow{4}{*}{$\mathrm{H}-4 \rightarrow \mathrm{L}+3(2 \%), \mathrm{H}-3 \rightarrow \mathrm{LUMO}(3 \%)$} \\
\hline & & $\mathrm{H}-2 \rightarrow \mathrm{L}+2(15 \%)$ & \\
\hline & & $\mathrm{H}-1 \rightarrow \mathrm{L}+1(18 \%)$ & \\
\hline & & $\mathrm{H}-1 \rightarrow \mathrm{L}+2(19 \%)$ & \\
\hline \multirow{3}{*}{317} & \multirow{3}{*}{0} & $\mathrm{H}-2 \rightarrow \mathrm{L}+1(15 \%)$ & \\
\hline & & $\mathrm{H}-1 \rightarrow \mathrm{L}+2(34 \%)$ & $\mathrm{H}-2 \rightarrow \mathrm{L}+2(6 \%)$ \\
\hline & & $\mathrm{HOMO} \rightarrow \mathrm{L}+3(38 \%)$ & \\
\hline \multirow{3}{*}{316} & \multirow{3}{*}{0.006} & $\mathrm{H}-4 \rightarrow \mathrm{L}+2(26 \%)$ & H-6 $\rightarrow$ LUMO (2\%), H-5 $\rightarrow$ LUMO (5\%), \\
\hline & & $\mathrm{H}-3 \rightarrow \mathrm{L}+1(23 \%)$ & $\mathrm{H}-4 \rightarrow \mathrm{L}+1(8 \%), \mathrm{H}-2 \rightarrow \mathrm{LUMO}(4 \%)$ \\
\hline & & $\mathrm{H}-3 \rightarrow \mathrm{L}+2(20 \%)$ & $\mathrm{H}-2 \rightarrow \mathrm{L}+3(5 \%)$ \\
\hline \multirow{3}{*}{315} & \multirow{3}{*}{0.005} & $\mathrm{H}-4 \rightarrow \mathrm{L}+1(38 \%)$ & H-6 $\rightarrow$ LUMO (5\%), H-5 $\rightarrow$ LUMO (2\%), \\
\hline & & $\mathrm{H}-3 \rightarrow \mathrm{L}+1(16 \%)$ & $\mathrm{H}-4 \rightarrow \mathrm{L}+2(9 \%), \mathrm{H}-1 \rightarrow \mathrm{LUMO}(4 \%)$ \\
\hline & & $\mathrm{H}-3 \rightarrow \mathrm{L}+2(13 \%)$ & $\mathrm{H}-1 \rightarrow \mathrm{L}+3(5 \%), \mathrm{HOMO} \rightarrow \mathrm{L}+8(2 \%)$ \\
\hline \multirow{4}{*}{313} & & $\mathrm{H}-4 \rightarrow \mathrm{L}+1(22 \%)$ & \\
\hline & 12 & $\mathrm{H}-4 \rightarrow \mathrm{L}+2(11 \%)$ & \\
\hline & 0.15 & $\mathrm{H}-3 \rightarrow \mathrm{L}+1(34 \%)$ & \\
\hline & & $\mathrm{H}-3 \rightarrow \mathrm{L}+2(26 \%)$ & \\
\hline 312 & 01 & $\mathrm{H}-6 \rightarrow \mathrm{L}+2(37 \%)$ & $\mathrm{H}-4 \rightarrow \mathrm{L}+2(4 \%), \mathrm{H}-4 \rightarrow \mathrm{L}+3(4 \%)$ \\
\hline & & $\mathrm{H}-5 \rightarrow \mathrm{L}+1(39 \%)$ & $\mathrm{H}-3 \rightarrow \mathrm{LUMO}(6 \%), \mathrm{H}-3 \rightarrow \mathrm{L}+1(3 \%)$ \\
\hline & & $\mathrm{H}-4 \rightarrow \mathrm{L}+1(14 \%)$ & \\
\hline 312 & 0.12 & $\mathrm{H}-4 \rightarrow \mathrm{L}+2(33 \%)$ & $\mathrm{H}-6 \rightarrow \mathrm{L}+2(2 \%), \mathrm{H}-5 \rightarrow \mathrm{L}+1(3 \%)$ \\
\hline & & $\mathrm{H}-3 \rightarrow \mathrm{L}+1(14 \%)$ & \\
\hline
\end{tabular}




\begin{tabular}{lll}
\hline & $\mathrm{H}-3 \rightarrow \mathrm{L}+2(28 \%)$ \\
\hline \multirow{2}{*}{308} & 0 & $\mathrm{H}-6 \rightarrow \mathrm{L}+1(46 \%)$ \\
& $\mathrm{H}-5 \rightarrow \mathrm{L}+2(45 \%)$ \\
\hline
\end{tabular}

Table S 11 TD-DFT results of [4]C-Bu-Cbz (b3lyp/6-311+g(d,p).

\begin{tabular}{|c|c|c|c|}
\hline$\lambda(\mathbf{n m})$ & $\begin{array}{l}\text { Oscillator } \\
\text { Strength }\end{array}$ & Major contributions & Minor contributions \\
\hline 430 & 0.00 & $\mathrm{HOMO} \rightarrow \mathrm{LUMO}(97 \%)$ & \\
\hline 362 & 0.14 & $\mathrm{H}-1 \rightarrow \mathrm{LUMO}(65 \%)$ & $\begin{array}{c}\mathrm{H}-5 \rightarrow \mathrm{LUMO}(7 \%), \mathrm{H}-3 \rightarrow \mathrm{L}+1(3 \%), \mathrm{H}-3 \rightarrow \mathrm{L}+2(3 \%), \\
\mathrm{HOMO} \rightarrow \mathrm{L}+1(9 \%), \mathrm{HOMO} \rightarrow \mathrm{L}+2(7 \%)\end{array}$ \\
\hline 361 & 0.17 & $\begin{array}{l}\mathrm{H}-2 \rightarrow \mathrm{LUMO}(63 \%) \\
\mathrm{HOMO} \rightarrow \mathrm{L}+2(10 \%)\end{array}$ & $\mathrm{H}-6 \rightarrow \mathrm{LUMO}(8 \%), \mathrm{HOMO} \rightarrow \mathrm{L}+1(8 \%)$ \\
\hline 358 & 0.01 & $\mathrm{H}-3 \rightarrow \mathrm{LUMO}(86 \%)$ & $\mathrm{H}-5 \rightarrow \mathrm{L}+1(2 \%)$ \\
\hline 357 & 0.06 & $\mathrm{H}-4 \rightarrow \mathrm{LUMO}(84 \%)$ & $\mathrm{H}-2 \rightarrow \mathrm{L}+1(2 \%), \mathrm{H}-2 \rightarrow \mathrm{L}+2(2 \%), \mathrm{HOMO} \rightarrow \mathrm{L}+5(2 \%)$ \\
\hline 354 & 0.15 & $\begin{array}{l}\mathrm{H}-1 \rightarrow \mathrm{LUMO}(15 \%) \\
\mathrm{HOMO} \rightarrow \mathrm{L}+1(74 \%)\end{array}$ & $\mathrm{H}-2 \rightarrow \mathrm{LUMO}(6 \%), \mathrm{HOMO} \rightarrow \mathrm{L}+2(2 \%)$ \\
\hline 354 & 0.15 & $\begin{array}{l}\mathrm{H}-2 \rightarrow \mathrm{LUMO}(16 \%) \\
\mathrm{HOMO} \rightarrow \mathrm{L}+2(75 \%)\end{array}$ & $\mathrm{H}-1 \rightarrow \mathrm{LUMO}(5 \%), \mathrm{HOMO} \rightarrow \mathrm{L}+1(2 \%)$ \\
\hline 340 & 0.48 & $\begin{array}{l}\mathrm{H}-6 \rightarrow \text { LUMO }(30 \%), \mathrm{H}- \\
\quad 5 \rightarrow \text { LUMO }(50 \%)\end{array}$ & $\begin{array}{c}\mathrm{H}-4 \rightarrow \mathrm{L}+2(4 \%), \mathrm{H}-2 \rightarrow \mathrm{LUMO}(7 \%) \\
\mathrm{H}-1 \rightarrow \mathrm{LUMO}(2 \%)\end{array}$ \\
\hline 340 & 0.47 & $\begin{aligned} \mathrm{H}-6 & \rightarrow \text { LUMO }(50 \%), \mathrm{H}- \\
5 & \rightarrow \text { LUMO }(31 \%)\end{aligned}$ & $\begin{array}{c}\mathrm{H}-4 \rightarrow \mathrm{L}+1(4 \%), \mathrm{H}-2 \rightarrow \mathrm{LUMO}(3 \%) \\
\mathrm{H}-1 \rightarrow \mathrm{LUMO}(6 \%)\end{array}$ \\
\hline 325 & 0.00 & $\begin{array}{c}\mathrm{H}-2 \rightarrow \mathrm{L}+1(10 \%), \\
\mathrm{H}-1 \rightarrow \mathrm{L}+1(10 \%), \\
\mathrm{H}-1 \rightarrow \mathrm{L}+2(11 \%), \\
\mathrm{HOMO} \rightarrow \mathrm{L}+3(52 \%)\end{array}$ & $\mathrm{H}-2 \rightarrow \mathrm{L}+2(9 \%)$ \\
\hline 322 & 0.01 & $\begin{array}{c}\mathrm{H}-4 \rightarrow \mathrm{LUMO}(11 \%), \mathrm{H}- \\
2 \rightarrow \mathrm{L}+1(19 \%), \\
\mathrm{H}-2 \rightarrow \mathrm{L}+2(16 \%), \\
\mathrm{H}-1 \rightarrow \mathrm{L}+1(25 \%), \\
\mathrm{H}-1 \rightarrow \mathrm{L}+2(18 \%)\end{array}$ & $\mathrm{H}-3 \rightarrow \mathrm{L}+3(5 \%)$ \\
\hline 320 & 0.00 & $\begin{array}{l}\mathrm{H}-2 \rightarrow \mathrm{L}+1(18 \%), \\
\mathrm{H}-2 \rightarrow \mathrm{L}+2(29 \%), \\
\mathrm{H}-1 \rightarrow \mathrm{L}+1(25 \%), \\
\mathrm{H}-1 \rightarrow \mathrm{L}+2(25 \%)\end{array}$ & \\
\hline
\end{tabular}

Table S 12 TD-DFT results of [4]C-Hex-Cbz (b3lyp/6-311+g(d,p).

\begin{tabular}{|c|c|c|c|}
\hline$\lambda(\mathbf{n m})$ & $\begin{array}{l}\text { Oscillator } \\
\text { Strength }\end{array}$ & Major contributions & Minor contributions \\
\hline 431 & 0.00 & $\begin{array}{c}\mathrm{HOMO} \rightarrow \text { LUMO } \\
(97 \%)\end{array}$ & \\
\hline
\end{tabular}




\begin{tabular}{|c|c|c|c|}
\hline 362 & 0.14 & $\begin{array}{l}\mathrm{H}-1 \rightarrow \mathrm{LUMO}(67 \%) \\
\mathrm{HOMO} \rightarrow \mathrm{L}+1(12 \%)\end{array}$ & $\begin{array}{c}\mathrm{H}-6 \rightarrow \mathrm{LUMO}(2 \%), \mathrm{H}-5 \rightarrow \mathrm{LUMO}(5 \%), \\
\mathrm{H}-3 \rightarrow \mathrm{L}+2(5 \%), \mathrm{HOMO} \rightarrow \mathrm{L}+2(2 \%)\end{array}$ \\
\hline 362 & 0.16 & $\begin{array}{l}\mathrm{H}-2 \rightarrow \mathrm{LUMO}(64 \%) \\
\mathrm{HOMO} \rightarrow \mathrm{L}+2(13 \%)\end{array}$ & $\begin{array}{c}\mathrm{H}-6 \rightarrow \mathrm{LUMO}(6 \%), \mathrm{H}-5 \rightarrow \mathrm{LUMO}(3 \%), \\
\mathrm{H}-4 \rightarrow \mathrm{L}+1(3 \%), \mathrm{H}-3 \rightarrow \mathrm{L}+1(2 \%), \mathrm{HOMO} \rightarrow \mathrm{L}+1 \\
(3 \%)\end{array}$ \\
\hline 359 & 0.01 & $\mathrm{H}-3 \rightarrow \mathrm{LUMO}(85 \%)$ & \\
\hline 357 & 0.07 & H-4 $\rightarrow$ LUMO (84\%) & $\mathrm{H}-2 \rightarrow \mathrm{L}+1(4 \%), \mathrm{H}-1 \underset{(2 \%)}{\rightarrow \mathrm{L}+2}(2 \%), \mathrm{HOMO} \rightarrow \mathrm{L}+5$ \\
\hline 354 & 0.15 & $\begin{array}{l}\mathrm{H}-1 \rightarrow \mathrm{LUMO}(17 \%) \\
\mathrm{HOMO} \rightarrow \mathrm{L}+1(76 \%)\end{array}$ & $\mathrm{HOMO} \rightarrow \mathrm{L}+2(2 \%)$ \\
\hline 354 & 0.15 & $\begin{array}{l}\mathrm{H}-2 \rightarrow \mathrm{LUMO}(19 \%) \\
\mathrm{HOMO} \rightarrow \mathrm{L}+2(75 \%)\end{array}$ & \\
\hline 340 & 0.47 & $\begin{array}{l}\text { H-6 } \rightarrow \text { LUMO }(33 \%) \\
\text { H-5 } \rightarrow \text { LUMO }(47 \%)\end{array}$ & $\mathrm{H}-4 \rightarrow \mathrm{L}+1(2 \%), \mathrm{H}-2 \rightarrow \mathrm{LUMO}(8 \%)$ \\
\hline 340 & 0.46 & $\begin{array}{l}\mathrm{H}-6 \rightarrow \text { LUMO }(48 \%) \\
\mathrm{H}-5 \rightarrow \text { LUMO }(34 \%)\end{array}$ & $\mathrm{H}-1 \rightarrow \mathrm{LUMO}(8 \%)$ \\
\hline 325 & 0.00 & $\begin{array}{c}\mathrm{H}-2 \rightarrow \mathrm{L}+2(14 \%) \\
\mathrm{H}-1 \rightarrow \mathrm{L}+1(19 \%) \\
\mathrm{HOMO} \rightarrow \mathrm{L}+3(51 \%)\end{array}$ & $\mathrm{H}-2 \rightarrow \mathrm{L}+1(4 \%), \mathrm{H}-1 \rightarrow \mathrm{L}+2(5 \%)$ \\
\hline 322 & 0.01 & $\begin{array}{c}\mathrm{H}-4 \rightarrow \mathrm{LUMO}(10 \%), \\
\mathrm{H}-2 \rightarrow \mathrm{L}+1(30 \%), \\
\mathrm{H}-1 \rightarrow \mathrm{L}+1(12 \%), \\
\mathrm{H}-1 \rightarrow \mathrm{L}+2(32 \%)\end{array}$ & $\mathrm{H}-3 \rightarrow \mathrm{L}+3(5 \%), \mathrm{H}-2 \rightarrow \mathrm{L}+2(6 \%)$ \\
\hline 320 & 0.00 & $\begin{array}{l}H-2 \rightarrow L+2(38 \%), \\
H-1 \rightarrow L+1(39 \%), \\
H-1 \rightarrow L+2(12 \%)\end{array}$ & $\mathrm{H}-2 \rightarrow \mathrm{L}+1(6 \%)$ \\
\hline
\end{tabular}

Table S 13 Atomic coordinates of [4]C-Et-Cbz after geometry optimization (b3lyp/6-31g(d))

\begin{tabular}{llll}
\hline Atom & $\mathrm{X}(\AA)$ & $\mathrm{Y}(\AA)$ & $\mathrm{Z}(\AA)$ \\
\hline C & -2.650892 & 4.261788 & 1.01839 \\
$\mathrm{C}$ & -3.711753 & 3.378971 & 1.223109 \\
$\mathrm{H}$ & -3.738279 & 2.746007 & 2.103337 \\
$\mathrm{C}$ & -4.59059 & 3.117319 & 0.161492 \\
$\mathrm{C}$ & -4.50676 & 3.912552 & -1.007301 \\
$\mathrm{H}$ & -5.22298 & 3.750001 & -1.808292 \\
C & -3.466955 & 4.819434 & -1.194471 \\
$\mathrm{H}$ & -3.38576 & 5.36034 & -2.134089 \\
$\mathrm{C}$ & -2.468039 & 4.941042 & -0.215479 \\
$\mathrm{C}$ & -1.093831 & 5.408487 & -0.222778 \\
$\mathrm{C}$ & -0.237471 & 5.91758 & -1.211812
\end{tabular}




\begin{tabular}{|c|c|c|c|}
\hline $\mathrm{H}$ & -0.640649 & 6.292934 & -2.149144 \\
\hline $\mathrm{C}$ & 1.14147 & 5.834433 & -1.037153 \\
\hline $\mathrm{H}$ & 1.800798 & 6.140202 & -1.845105 \\
\hline $\mathrm{C}$ & 1.704005 & 5.260211 & 0.128764 \\
\hline $\mathrm{C}$ & 0.858069 & 4.934896 & 1.199299 \\
\hline $\mathrm{H}$ & 1.273192 & 4.451838 & 2.077065 \\
\hline $\mathrm{C}$ & -0.522999 & 4.986266 & 1.007293 \\
\hline $\mathrm{C}$ & -1.25861 & 3.692752 & 3.045927 \\
\hline $\mathrm{H}$ & -2.186304 & 3.707239 & 3.627628 \\
\hline $\mathrm{H}$ & -0.526978 & 4.272858 & 3.618144 \\
\hline $\mathrm{C}$ & -0.768397 & 2.251121 & 2.854207 \\
\hline $\mathrm{H}$ & -1.50248 & 1.654025 & 2.304718 \\
\hline $\mathrm{H}$ & -0.603509 & 1.779475 & 3.829755 \\
\hline $\mathrm{H}$ & 0.173309 & 2.224611 & 2.297498 \\
\hline $\mathrm{C}$ & 4.214071 & 2.710864 & -0.795184 \\
\hline $\mathrm{C}$ & 3.329154 & 3.777361 & -0.961094 \\
\hline $\mathrm{H}$ & 2.706605 & 3.846543 & -1.846564 \\
\hline $\mathrm{C}$ & 3.045753 & 4.604071 & 0.135339 \\
\hline $\mathrm{C}$ & 3.822475 & 4.471551 & 1.312224 \\
\hline $\mathrm{H}$ & 3.64424 & 5.150451 & 2.141808 \\
\hline $\mathrm{C}$ & 4.729851 & 3.42764 & 1.464802 \\
\hline $\mathrm{H}$ & 5.255929 & 3.303357 & 2.408126 \\
\hline $\mathrm{C}$ & 4.873389 & 2.476364 & 0.441857 \\
\hline $\mathrm{C}$ & 5.343904 & 1.104567 & 0.402171 \\
\hline $\mathrm{C}$ & 5.825604 & 0.207651 & 1.369054 \\
\hline $\mathrm{H}$ & 6.179577 & 0.572311 & 2.330169 \\
\hline $\mathrm{C}$ & 5.741661 & -1.162783 & 1.138991 \\
\hline $\mathrm{H}$ & 6.028336 & -1.854329 & 1.926726 \\
\hline $\mathrm{C}$ & 5.190295 & -1.677089 & -0.060148 \\
\hline $\mathrm{C}$ & 4.895784 & -0.789124 & -1.104842 \\
\hline $\mathrm{H}$ & 4.434498 & -1.167631 & -2.010816 \\
\hline $\mathrm{C}$ & 4.947945 & 0.583709 & -0.857973 \\
\hline $\mathrm{C}$ & 3.782255 & 1.402172 & -2.934311 \\
\hline $\mathrm{H}$ & 3.094388 & 2.230027 & -3.120428 \\
\hline $\mathrm{H}$ & 3.173535 & 0.488565 & -2.915652 \\
\hline
\end{tabular}




\begin{tabular}{|c|c|c|c|}
\hline $\mathrm{C}$ & 4.826169 & 1.325833 & -4.052684 \\
\hline $\mathrm{H}$ & 5.404888 & 2.253638 & -4.10579 \\
\hline $\mathrm{H}$ & 4.335206 & 1.166817 & -5.019576 \\
\hline $\mathrm{H}$ & 5.524618 & 0.499867 & -3.884088 \\
\hline $\mathrm{C}$ & 2.623407 & -4.217236 & 0.717975 \\
\hline $\mathrm{C}$ & 3.689221 & -3.343957 & 0.940533 \\
\hline $\mathrm{H}$ & 3.744177 & -2.766667 & 1.857431 \\
\hline $\mathrm{C}$ & 4.530259 & -3.014513 & -0.13226 \\
\hline $\mathrm{C}$ & 4.410505 & -3.740931 & -1.34239 \\
\hline $\mathrm{H}$ & 5.101379 & -3.530062 & -2.154277 \\
\hline $\mathrm{C}$ & 3.366796 & -4.638763 & -1.549193 \\
\hline $\mathrm{H}$ & 3.255276 & -5.122113 & -2.516647 \\
\hline $\mathrm{C}$ & 2.401399 & -4.823509 & -0.546509 \\
\hline $\mathrm{C}$ & 1.026947 & -5.287921 & -0.539528 \\
\hline $\mathrm{C}$ & 0.145094 & -5.736008 & -1.53605 \\
\hline $\mathrm{H}$ & 0.526104 & -6.057059 & -2.502393 \\
\hline $\mathrm{C}$ & -1.228385 & -5.661739 & -1.324859 \\
\hline $\mathrm{H}$ & -1.908192 & -5.921532 & -2.131872 \\
\hline $\mathrm{C}$ & -1.759362 & -5.153036 & -0.114428 \\
\hline $\mathrm{C}$ & -0.887338 & -4.8912 & 0.951717 \\
\hline $\mathrm{H}$ & -1.286009 & -4.459109 & 1.863153 \\
\hline $\mathrm{C}$ & 0.489613 & -4.934222 & 0.7279 \\
\hline $\mathrm{C}$ & 1.349268 & -3.835032 & 2.855652 \\
\hline $\mathrm{H}$ & 0.281814 & -3.792793 & 3.083678 \\
\hline $\mathrm{H}$ & 1.707402 & -2.797227 & 2.844311 \\
\hline $\mathrm{C}$ & -4.294105 & -2.560185 & -0.828155 \\
\hline $\mathrm{C}$ & -3.412661 & -3.613588 & -1.076492 \\
\hline $\mathrm{H}$ & -2.813348 & -3.631525 & -1.980315 \\
\hline $\mathrm{C}$ & -3.100115 & -4.499678 & -0.035828 \\
\hline $\mathrm{C}$ & -3.845924 & -4.435037 & 1.166484 \\
\hline $\mathrm{H}$ & -3.645856 & -5.159422 & 1.951426 \\
\hline $\mathrm{C}$ & -4.748941 & -3.402685 & 1.401458 \\
\hline $\mathrm{H}$ & -5.249643 & -3.33188 & 2.36392 \\
\hline $\mathrm{C}$ & -4.919712 & -2.39624 & 0.437431 \\
\hline $\mathrm{C}$ & -5.392117 & -1.025442 & 0.486904 \\
\hline
\end{tabular}




\begin{tabular}{|c|c|c|c|}
\hline $\mathrm{C}$ & -5.846686 & -0.184507 & 1.51525 \\
\hline $\mathrm{H}$ & -6.173366 & -0.602589 & 2.464228 \\
\hline $\mathrm{C}$ & -5.769204 & 1.196804 & 1.359552 \\
\hline $\mathrm{H}$ & -6.033519 & 1.843039 & 2.19231 \\
\hline $\mathrm{C}$ & -5.250996 & 1.777886 & 0.176214 \\
\hline $\mathrm{C}$ & -4.985144 & 0.949811 & -0.92391 \\
\hline $\mathrm{H}$ & -4.547187 & 1.378436 & -1.819032 \\
\hline $\mathrm{C}$ & -5.031535 & -0.434769 & -0.752836 \\
\hline $\mathrm{C}$ & -3.928518 & -1.135265 & -2.904973 \\
\hline $\mathrm{H}$ & -3.264652 & -1.962006 & -3.167034 \\
\hline $\mathrm{H}$ & -3.301937 & -0.234947 & -2.851571 \\
\hline $\mathrm{C}$ & -5.009588 & -0.970862 & -3.977826 \\
\hline $\mathrm{H}$ & -5.606985 & -1.883855 & -4.067502 \\
\hline $\mathrm{H}$ & -4.550145 & -0.758899 & -4.949998 \\
\hline $\mathrm{H}$ & -5.685881 & -0.145897 & -3.732172 \\
\hline $\mathrm{N}$ & -1.510238 & 4.410765 & 1.803998 \\
\hline $\mathrm{N}$ & 4.374851 & 1.598182 & -1.620176 \\
\hline $\mathrm{N}$ & 1.501637 & -4.397453 & 1.522699 \\
\hline $\mathrm{N}$ & -4.479778 & -1.404406 & -1.585888 \\
\hline $\mathrm{C}$ & 2.084723 & -4.64235 & 3.929773 \\
\hline $\mathrm{H}$ & 3.158044 & -4.690973 & 3.719615 \\
\hline $\mathrm{H}$ & 1.949189 & -4.177731 & 4.913121 \\
\hline $\mathrm{H}$ & 1.701105 & -5.666754 & 3.973993 \\
\hline
\end{tabular}

Number of imaginary frequency: 0

Table S14 Atomic coordinates of [2]L-Et-Cbz after geometry optimization (b3lyp/6-31g(d))

\begin{tabular}{llll}
\hline Atom & $\mathrm{X}(\AA)$ & $\mathrm{Y}(\AA)$ & $\mathrm{Z}(\AA)$ \\
\hline C & 6.634592 & 0.523154 & 0.539932 \\
$\mathrm{C}$ & 5.284498 & 0.235801 & 0.315914 \\
$\mathrm{C}$ & 4.866888 & -1.035318 & -0.164316 \\
$\mathrm{C}$ & 5.822927 & -2.027137 & -0.411496 \\
$\mathrm{C}$ & 7.167711 & -1.746168 & -0.185357 \\
$\mathrm{C}$ & 7.565008 & -0.482808 & 0.285179 \\
$\mathrm{H}$ & 6.957697 & 1.496459 & 0.897328 \\
$\mathrm{H}$ & 5.518564 & -3.004592 & -0.777113
\end{tabular}




\begin{tabular}{|c|c|c|c|}
\hline $\mathrm{H}$ & 7.9184 & -2.508018 & -0.375264 \\
\hline $\mathrm{H}$ & 8.619904 & -0.282067 & 0.452276 \\
\hline $\mathrm{C}$ & 3.426688 & -0.976831 & -0.284845 \\
\hline $\mathrm{C}$ & 2.441086 & -1.884544 & -0.68736 \\
\hline $\mathrm{C}$ & 3.035185 & 0.325755 & 0.123391 \\
\hline $\mathrm{C}$ & 1.107662 & -1.493377 & -0.680343 \\
\hline $\mathrm{H}$ & 2.713838 & -2.884766 & -1.014354 \\
\hline $\mathrm{C}$ & 1.697319 & 0.723497 & 0.127458 \\
\hline $\mathrm{C}$ & 0.716706 & -0.1947 & -0.275475 \\
\hline $\mathrm{H}$ & 0.346251 & -2.186713 & -1.02447 \\
\hline $\mathrm{H}$ & 1.405111 & 1.713949 & 0.461755 \\
\hline $\mathrm{C}$ & 4.185954 & 2.433589 & 0.924414 \\
\hline $\mathrm{C}$ & 4.316585 & 3.425286 & -0.237019 \\
\hline $\mathrm{H}$ & 3.478212 & 3.322351 & -0.93372 \\
\hline $\mathrm{H}$ & 4.326453 & 4.454338 & 0.140202 \\
\hline $\mathrm{H}$ & 5.243284 & 3.253801 & -0.794212 \\
\hline $\mathrm{N}$ & 4.166274 & 1.042957 & 0.501015 \\
\hline $\mathrm{H}$ & 3.268145 & 2.624984 & 1.490642 \\
\hline $\mathrm{H}$ & 5.015059 & 2.55691 & 1.629444 \\
\hline $\mathrm{C}$ & -0.716663 & 0.194785 & -0.275461 \\
\hline $\mathrm{C}$ & -1.107657 & 1.49343 & -0.680391 \\
\hline $\mathrm{C}$ & -1.697256 & -0.723432 & 0.127477 \\
\hline $\mathrm{C}$ & -2.441092 & 1.884563 & -0.687412 \\
\hline $\mathrm{H}$ & -0.346271 & 2.186767 & -1.024569 \\
\hline $\mathrm{C}$ & -3.035132 & -0.325735 & 0.123384 \\
\hline $\mathrm{H}$ & -1.405016 & -1.713867 & 0.461805 \\
\hline $\mathrm{C}$ & -3.42667 & 0.976838 & -0.284877 \\
\hline $\mathrm{H}$ & -2.71387 & 2.884772 & -1.014425 \\
\hline $\mathrm{C}$ & -4.866866 & 1.035314 & -0.164284 \\
\hline $\mathrm{C}$ & -5.28445 & -0.235829 & 0.315887 \\
\hline $\mathrm{C}$ & -5.822922 & 2.027132 & -0.411416 \\
\hline $\mathrm{C}$ & -6.63454 & -0.523228 & 0.539877 \\
\hline $\mathrm{C}$ & -7.167699 & 1.746127 & -0.185289 \\
\hline $\mathrm{H}$ & -5.518578 & 3.004603 & -0.777004 \\
\hline $\mathrm{C}$ & -7.564974 & 0.482733 & 0.285178 \\
\hline
\end{tabular}




\begin{tabular}{llll}
$\mathrm{H}$ & -6.957623 & -1.496563 & 0.897205 \\
$\mathrm{H}$ & -7.918405 & 2.507972 & -0.375151 \\
$\mathrm{H}$ & -8.619868 & 0.281968 & 0.452259 \\
$\mathrm{C}$ & -4.185917 & -2.433595 & 0.924397 \\
$\mathrm{H}$ & -3.267996 & -2.625074 & 1.490427 \\
$\mathrm{H}$ & -5.014867 & -2.556835 & 1.629618 \\
$\mathrm{C}$ & -4.316914 & -3.425279 & -0.237001 \\
$\mathrm{H}$ & -5.243646 & -3.253578 & -0.794085 \\
$\mathrm{H}$ & -3.47861 & -3.32256 & -0.933806 \\
$\mathrm{H}$ & -4.327008 & -4.454322 & 0.140236 \\
$\mathrm{~N}$ & -4.166203 & -1.042962 & 0.500999 \\
\hline
\end{tabular}

Number of imaginary frequency: 0

Table S15 Atomic coordinates of [3]L-Et-Cbz after geometry optimization (b3lyp/6-31g(d))

\begin{tabular}{llll}
\hline Atom & $\mathrm{X}(\AA)$ & $\mathrm{Y}(\AA)$ & $\mathrm{Z}(\AA)$ \\
\hline $\mathrm{C}$ & -10.789945 & 0.432131 & -0.665296 \\
$\mathrm{C}$ & -9.443891 & 0.188106 & -0.375565 \\
$\mathrm{C}$ & -9.044157 & -0.932854 & 0.401828 \\
$\mathrm{C}$ & -10.014203 & -1.820244 & 0.88193 \\
$\mathrm{C}$ & -11.355053 & -1.583324 & 0.591267 \\
$\mathrm{C}$ & -11.734537 & -0.467344 & -0.174233 \\
$\mathrm{H}$ & -11.099446 & 1.293566 & -1.24969 \\
$\mathrm{H}$ & -9.723555 & -2.68277 & 1.476263 \\
$\mathrm{H}$ & -12.116614 & -2.264536 & 0.960026 \\
$\mathrm{H}$ & -12.786691 & -0.297935 & -0.38707 \\
$\mathrm{C}$ & -7.603209 & -0.866278 & 0.508954 \\
$\mathrm{C}$ & -6.630705 & -1.660292 & 1.126271 \\
$\mathrm{C}$ & -7.193428 & 0.291227 & -0.204514 \\
$\mathrm{C}$ & -5.292064 & -1.30009 & 1.030083 \\
$\mathrm{H}$ & -6.917797 & -2.545702 & 1.687832 \\
$\mathrm{C}$ & -5.850148 & 0.657695 & -0.30067 \\
$\mathrm{C}$ & -4.882644 & -0.145635 & 0.320663 \\
$\mathrm{H}$ & -4.541267 & -1.896859 & 1.538843 \\
$\mathrm{H}$ & -5.54384 & 1.531341 & -0.867237 \\
$\mathrm{C}$ & -8.314603 & 2.157701 & -1.495289 \\
& & &
\end{tabular}




\begin{tabular}{|c|c|c|c|}
\hline $\mathrm{C}$ & -8.424564 & 3.400946 & -0.605333 \\
\hline $\mathrm{H}$ & -7.586273 & 3.452224 & 0.097 \\
\hline $\mathrm{H}$ & -8.417174 & 4.309929 & -1.21772 \\
\hline $\mathrm{H}$ & -9.352793 & 3.384056 & -0.024924 \\
\hline $\mathrm{N}$ & -8.314402 & 0.909438 & -0.74988 \\
\hline $\mathrm{H}$ & -7.396537 & 2.192684 & -2.091643 \\
\hline $\mathrm{H}$ & -9.144834 & 2.121821 & -2.208634 \\
\hline $\mathrm{C}$ & -3.444545 & 0.215208 & 0.234093 \\
\hline $\mathrm{C}$ & -3.040442 & 1.570252 & 0.300054 \\
\hline $\mathrm{C}$ & -2.472291 & -0.78519 & 0.086209 \\
\hline $\mathrm{C}$ & -1.702715 & 1.936921 & 0.219609 \\
\hline $\mathrm{H}$ & -3.795239 & 2.336007 & 0.450634 \\
\hline $\mathrm{C}$ & -1.129735 & -0.413724 & 0.002413 \\
\hline $\mathrm{H}$ & -2.774792 & -1.824808 & 0.010285 \\
\hline $\mathrm{C}$ & -0.725518 & 0.946706 & 0.069753 \\
\hline $\mathrm{H}$ & -1.419763 & 2.984519 & 0.28451 \\
\hline $\mathrm{C}$ & 0.714517 & 0.957533 & -0.047771 \\
\hline $\mathrm{C}$ & 1.121528 & -0.396388 & -0.187858 \\
\hline $\mathrm{C}$ & 1.689946 & 1.960827 & -0.047303 \\
\hline $\mathrm{C}$ & 2.465813 & -0.750117 & -0.311333 \\
\hline $\mathrm{C}$ & 3.028901 & 1.612198 & -0.175619 \\
\hline $\mathrm{H}$ & 1.405347 & 3.005991 & 0.044141 \\
\hline $\mathrm{C}$ & 3.43627 & 0.26293 & -0.306857 \\
\hline $\mathrm{H}$ & 2.771144 & -1.789664 & -0.375371 \\
\hline $\mathrm{H}$ & 3.782628 & 2.393066 & -0.206554 \\
\hline $\mathrm{C}$ & 0.005535 & -2.66728 & -0.219017 \\
\hline $\mathrm{H}$ & -0.919594 & -2.992624 & -0.706327 \\
\hline $\mathrm{H}$ & 0.825992 & -2.97437 & -0.876499 \\
\hline $\mathrm{C}$ & 0.147278 & -3.324146 & 1.158674 \\
\hline $\mathrm{H}$ & 1.081465 & -3.019306 & 1.641243 \\
\hline $\mathrm{H}$ & -0.682257 & -3.038622 & 1.813718 \\
\hline $\mathrm{H}$ & 0.149873 & -4.41584 & 1.061524 \\
\hline $\mathrm{N}$ & -0.00431 & -1.214064 & -0.169862 \\
\hline $\mathrm{C}$ & 4.875415 & -0.081035 & -0.43425 \\
\hline $\mathrm{C}$ & 5.838833 & 0.623015 & 0.302958 \\
\hline
\end{tabular}




\begin{tabular}{|c|c|c|c|}
\hline $\mathrm{C}$ & 5.289474 & -1.121363 & -1.300349 \\
\hline $\mathrm{C}$ & 7.182655 & 0.272303 & 0.164325 \\
\hline $\mathrm{H}$ & 5.529258 & 1.406053 & 0.987761 \\
\hline $\mathrm{C}$ & 6.628674 & -1.465034 & -1.439053 \\
\hline $\mathrm{H}$ & 4.541685 & -1.638435 & -1.893843 \\
\hline $\mathrm{C}$ & 7.597113 & -0.769841 & -0.706816 \\
\hline $\mathrm{H}$ & 6.919159 & -2.260471 & -2.120703 \\
\hline $\mathrm{C}$ & 9.037104 & -0.853573 & -0.600011 \\
\hline $\mathrm{C}$ & 10.010016 & -1.663231 & -1.197309 \\
\hline $\mathrm{C}$ & 9.431745 & 0.141231 & 0.335614 \\
\hline $\mathrm{C}$ & 11.348818 & -1.473807 & -0.865413 \\
\hline $\mathrm{H}$ & 9.723339 & -2.429388 & -1.913224 \\
\hline $\mathrm{C}$ & 10.775782 & 0.337384 & 0.668051 \\
\hline $\mathrm{C}$ & 11.723377 & -0.482571 & 0.057891 \\
\hline $\mathrm{H}$ & 12.11257 & -2.095306 & -1.324136 \\
\hline $\mathrm{H}$ & 11.081565 & 1.103363 & 1.374546 \\
\hline $\mathrm{H}$ & 12.773988 & -0.348178 & 0.301131 \\
\hline $\mathrm{C}$ & 8.295664 & 1.929079 & 1.72138 \\
\hline $\mathrm{H}$ & 7.372746 & 1.879536 & 2.309113 \\
\hline $\mathrm{H}$ & 9.119905 & 1.787289 & 2.42855 \\
\hline $\mathrm{C}$ & 8.414874 & 3.28849 & 1.023257 \\
\hline $\mathrm{H}$ & 7.581251 & 3.445472 & 0.331047 \\
\hline $\mathrm{H}$ & 8.406216 & 4.098066 & 1.762073 \\
\hline $\mathrm{H}$ & 9.346639 & 3.353352 & 0.452028 \\
\hline $\mathrm{N}$ & 8.299869 & 0.802532 & 0.802428 \\
\hline
\end{tabular}

Number of imaginary frequency: 0

Table S 16 Atomic coordinates of [4]C-Bu-Cbz after geometry optimization (b3lyp/6-31g(d))

\begin{tabular}{llll}
\hline Atom & $\mathbf{X}(\AA)$ & $\mathbf{Y}(\AA)$ & $\mathbf{Z}(\AA)$ \\
\hline $\mathrm{C}$ & -4.57772800 & -2.18422200 & -0.76055000 \\
$\mathrm{C}$ & -3.83321400 & -3.34912200 & -0.95396500 \\
$\mathrm{C}$ & -3.62100700 & -4.20660300 & 0.13529300 \\
$\mathrm{C}$ & -4.34504900 & -3.98316500 & 1.33202300 \\
$\mathrm{C}$ & -5.11458100 & -2.83676900 & 1.51057600 \\
$\mathrm{C}$ & -5.16904100 & -1.87210100 & 0.49198400
\end{tabular}




\begin{tabular}{|c|c|c|c|}
\hline $\mathrm{C}$ & -5.46333800 & -0.45192500 & 0.46057900 \\
\hline $\mathrm{C}$ & -5.81385000 & 0.49162100 & 1.43952000 \\
\hline $\mathrm{C}$ & -5.56888200 & 1.84255300 & 1.21298900 \\
\hline $\mathrm{C}$ & -4.98185700 & 2.29033100 & 0.00434800 \\
\hline $\mathrm{C}$ & -4.81507300 & 1.37819900 & -1.04740100 \\
\hline $\mathrm{C}$ & -5.03044500 & 0.02004700 & -0.80815700 \\
\hline $\mathrm{C}$ & -4.00466200 & -0.99810200 & -2.90488500 \\
\hline $\mathrm{C}$ & -4.84337000 & -1.67850000 & -3.99813800 \\
\hline $\mathrm{C}$ & -6.19352000 & -1.00043500 & -4.25902000 \\
\hline $\mathrm{C}$ & -7.01286400 & -1.70513600 & -5.34480800 \\
\hline $\mathrm{C}$ & -2.10886600 & 4.49722100 & 0.73220100 \\
\hline $\mathrm{C}$ & -3.26631400 & 3.75570300 & 0.97538100 \\
\hline $\mathrm{C}$ & -4.16595500 & 3.53832100 & -0.07829300 \\
\hline $\mathrm{C}$ & -3.98797300 & 4.25414100 & -1.28749300 \\
\hline $\mathrm{C}$ & -2.84839400 & 5.02080100 & -1.51525800 \\
\hline $\mathrm{C}$ & -1.84503400 & 5.08053600 & -0.53510100 \\
\hline $\mathrm{C}$ & -0.42461300 & 5.37428000 & -0.56027100 \\
\hline $\mathrm{C}$ & 0.48050700 & 5.71889700 & -1.57681700 \\
\hline $\mathrm{C}$ & 1.83924800 & 5.47540100 & -1.40078700 \\
\hline $\mathrm{C}$ & 2.33324500 & 4.89569200 & -0.20672500 \\
\hline $\mathrm{C}$ & 1.46226200 & 4.73515800 & 0.88022100 \\
\hline $\mathrm{C}$ & 0.09583700 & 4.94895600 & 0.69195200 \\
\hline $\mathrm{C}$ & -0.84090100 & 3.93614300 & 2.83242500 \\
\hline $\mathrm{C}$ & -1.47659700 & 4.78267000 & 3.94636200 \\
\hline $\mathrm{C}$ & -0.78624900 & 6.13280400 & 4.17291600 \\
\hline $\mathrm{C}$ & -1.44698800 & 6.96006700 & 5.28012400 \\
\hline $\mathrm{C}$ & 4.50865800 & 2.01553900 & -1.00134800 \\
\hline $\mathrm{C}$ & 3.75695600 & 3.17120700 & -1.21963400 \\
\hline $\mathrm{C}$ & 3.58424700 & 4.08081200 & -0.16672400 \\
\hline $\mathrm{C}$ & 4.34855400 & 3.91517100 & 1.01408000 \\
\hline $\mathrm{C}$ & 5.12417800 & 2.77804000 & 1.21850900 \\
\hline $\mathrm{C}$ & 5.14402100 & 1.76622100 & 0.24523400 \\
\hline $\mathrm{C}$ & 5.44628900 & 0.34769400 & 0.27492900 \\
\hline $\mathrm{C}$ & 5.82380300 & -0.55029000 & 1.28597500 \\
\hline $\mathrm{C}$ & 5.57313200 & -1.91045200 & 1.12693800 \\
\hline
\end{tabular}




\begin{tabular}{|c|c|c|c|}
\hline $\mathrm{C}$ & 4.95350100 & -2.41452200 & -0.04281700 \\
\hline $\mathrm{C}$ & 4.76061700 & -1.55181300 & -1.13160900 \\
\hline $\mathrm{C}$ & 4.98144500 & -0.18459500 & -0.95654800 \\
\hline $\mathrm{C}$ & 3.89634400 & 0.65633100 & -3.06590600 \\
\hline $\mathrm{C}$ & 4.89416700 & 0.36481500 & -4.19771200 \\
\hline $\mathrm{C}$ & 5.84405500 & 1.52843400 & -4.50499500 \\
\hline $\mathrm{C}$ & 6.83569600 & 1.20594600 & -5.62726000 \\
\hline $\mathrm{C}$ & 2.09945500 & -4.57464600 & 0.87067300 \\
\hline $\mathrm{C}$ & 3.26595100 & -3.82826100 & 1.04524000 \\
\hline $\mathrm{C}$ & 4.13401200 & -3.66272700 & -0.04364100 \\
\hline $\mathrm{C}$ & 3.91789700 & -4.43233800 & -1.21310000 \\
\hline $\mathrm{C}$ & 2.77077400 & -5.20519900 & -1.37068000 \\
\hline $\mathrm{C}$ & 1.79711300 & -5.21676500 & -0.35913800 \\
\hline $\mathrm{C}$ & 0.37705300 & -5.51121000 & -0.32654700 \\
\hline $\mathrm{C}$ & -0.55920600 & -5.90325600 & -1.29676800 \\
\hline $\mathrm{C}$ & -1.91234500 & -5.65395400 & -1.08878400 \\
\hline $\mathrm{C}$ & -2.37007400 & -5.02114100 & 0.09282000 \\
\hline $\mathrm{C}$ & -1.46533400 & -4.80931100 & 1.14277200 \\
\hline $\mathrm{C}$ & -0.10491500 & -5.02809800 & 0.92014000 \\
\hline $\mathrm{C}$ & 0.89347000 & -3.93328000 & 2.98708300 \\
\hline $\mathrm{C}$ & 1.49030500 & -4.78734500 & 4.11495900 \\
\hline $\mathrm{C}$ & 1.41520100 & -4.09351700 & 5.48057200 \\
\hline $\mathrm{C}$ & 2.00997700 & -4.93733000 & 6.61259000 \\
\hline $\mathrm{H}$ & -3.25349000 & -3.48632400 & -1.86046100 \\
\hline $\mathrm{H}$ & -4.22944300 & -4.68164400 & 2.15649400 \\
\hline $\mathrm{H}$ & -5.59386700 & -2.65301100 & 2.46899600 \\
\hline $\mathrm{H}$ & -6.19148700 & 0.16608600 & 2.40568100 \\
\hline $\mathrm{H}$ & -5.75356000 & 2.55991900 & 2.00806700 \\
\hline $\mathrm{H}$ & -4.32511200 & 1.70780300 & -1.95700000 \\
\hline $\mathrm{H}$ & -3.00688000 & -1.45418200 & -2.85572300 \\
\hline $\mathrm{H}$ & -3.85539200 & 0.05535200 & -3.15590600 \\
\hline $\mathrm{H}$ & -5.00502800 & -2.73003400 & -3.72549200 \\
\hline $\mathrm{H}$ & -4.24705100 & -1.68581300 & -4.92176800 \\
\hline $\mathrm{H}$ & -6.76921300 & -0.96992100 & -3.32550700 \\
\hline $\mathrm{H}$ & -6.02236100 & 0.04631100 & -4.54915300 \\
\hline
\end{tabular}




\begin{tabular}{|c|c|c|c|}
\hline $\mathrm{H}$ & -7.23026600 & -2.74325500 & -5.06532100 \\
\hline $\mathrm{H}$ & -6.47500900 & -1.72542100 & -6.30083600 \\
\hline $\mathrm{H}$ & -7.96995400 & -1.19858000 & -5.51187300 \\
\hline $\mathrm{H}$ & -3.36918700 & 3.18213100 & 1.89029700 \\
\hline $\mathrm{H}$ & -4.71812000 & 4.13451500 & -2.08345400 \\
\hline $\mathrm{H}$ & -2.70134400 & 5.49376100 & -2.48310700 \\
\hline $\mathrm{H}$ & 0.11798600 & 6.09086200 & -2.53195300 \\
\hline $\mathrm{H}$ & 2.52569000 & 5.65570600 & -2.22370300 \\
\hline $\mathrm{H}$ & 1.82690000 & 4.25096400 & 1.77949300 \\
\hline $\mathrm{H}$ & 0.22134300 & 3.78632400 & 3.04283700 \\
\hline $\mathrm{H}$ & -1.30017800 & 2.93893500 & 2.80728200 \\
\hline $\mathrm{H}$ & -1.44926900 & 4.19173800 & 4.87308000 \\
\hline $\mathrm{H}$ & -2.53759100 & 4.94511500 & 3.71376700 \\
\hline $\mathrm{H}$ & 0.27053200 & 5.96098100 & 4.42358400 \\
\hline $\mathrm{H}$ & -0.79048900 & 6.70294800 & 3.23551700 \\
\hline $\mathrm{H}$ & -1.43173400 & 6.42777600 & 6.23935800 \\
\hline $\mathrm{H}$ & -2.49459400 & 7.17834800 & 5.03941500 \\
\hline $\mathrm{H}$ & -0.93232100 & 7.91689000 & 5.42204000 \\
\hline $\mathrm{H}$ & 3.14094300 & 3.27019200 & -2.10667400 \\
\hline $\mathrm{H}$ & 4.26080100 & 4.65220900 & 1.80779200 \\
\hline $\mathrm{H}$ & 5.63847200 & 2.63857600 & 2.16620000 \\
\hline $\mathrm{H}$ & 6.22608000 & -0.18257300 & 2.22672800 \\
\hline $\mathrm{H}$ & 5.77998500 & -2.59051100 & 1.94895000 \\
\hline $\mathrm{H}$ & 4.24983200 & -1.91675500 & -2.01620400 \\
\hline $\mathrm{H}$ & 3.32524000 & 1.55859400 & -3.30006100 \\
\hline $\mathrm{H}$ & 3.16980600 & -0.16293000 & -2.98267500 \\
\hline $\mathrm{H}$ & 5.47729100 & -0.52991200 & -3.94151400 \\
\hline $\mathrm{H}$ & 4.31542400 & 0.11062100 & -5.09726400 \\
\hline $\mathrm{H}$ & 5.25351400 & 2.41434600 & -4.77986900 \\
\hline $\mathrm{H}$ & 6.39380100 & 1.79661800 & -3.59414000 \\
\hline $\mathrm{H}$ & 6.31540100 & 0.96210300 & -6.56188400 \\
\hline $\mathrm{H}$ & 7.46467300 & 0.34657200 & -5.36471700 \\
\hline $\mathrm{H}$ & 7.49922600 & 2.05464600 & -5.82718800 \\
\hline $\mathrm{H}$ & 3.39772300 & -3.21389700 & 1.92954500 \\
\hline $\mathrm{H}$ & 4.62361600 & -4.35152000 & -2.03552000 \\
\hline
\end{tabular}




\begin{tabular}{llll}
$\mathrm{H}$ & 2.59430100 & -5.72269600 & -2.31040800 \\
$\mathrm{H}$ & -0.22641400 & -6.31868500 & -2.24477200 \\
$\mathrm{H}$ & -2.62394000 & -5.87262700 & -1.88042300 \\
$\mathrm{H}$ & -1.80244200 & -4.28551400 & 2.03065500 \\
$\mathrm{H}$ & -0.15723300 & -3.71816200 & 3.19960900 \\
$\mathrm{H}$ & 1.40440300 & -2.96232000 & 2.93542100 \\
$\mathrm{H}$ & 0.95765900 & -5.74690500 & 4.15053600 \\
$\mathrm{H}$ & 2.53563900 & -5.02191900 & 3.87385600 \\
$\mathrm{H}$ & 0.36669900 & -3.85738100 & 5.71256400 \\
$\mathrm{H}$ & 1.93988700 & -3.12871700 & 5.42890700 \\
$\mathrm{H}$ & 3.06753100 & -5.16028900 & 6.42656200 \\
$\mathrm{H}$ & 1.48287900 & -5.89380600 & 6.71244800 \\
$\mathrm{H}$ & 1.94276600 & -4.41719500 & 7.57462800 \\
$\mathrm{~N}$ & -4.61387800 & -1.06167800 & -1.58430900 \\
$\mathrm{~N}$ & -0.95524400 & 4.53734600 & 1.51162500 \\
$\mathrm{~N}$ & 4.53123100 & 0.85198700 & -1.77062500 \\
$\mathrm{~N}$ & 0.96911400 & -4.57282100 & 1.68346600 \\
\hline $\mathrm{N}$ & & & \\
\hline
\end{tabular}

Number of imaginary frequency: 0

Table S 17 Atomic coordinates of [2]L-Bu-Cbz after geometry optimization (b3lyp/6-31g(d))

\begin{tabular}{|c|c|c|c|}
\hline Atom & $\mathbf{X}(\AA)$ & $Y(\AA)$ & $\mathrm{Z}(\AA)$ \\
\hline $\mathrm{C}$ & -5.26824800 & -0.48102900 & 0.55098900 \\
\hline $\mathrm{C}$ & -6.64187000 & -0.38771700 & 0.79771300 \\
\hline $\mathrm{C}$ & -7.42338000 & -1.51744600 & 0.56187700 \\
\hline $\mathrm{C}$ & -6.85755000 & -2.71371900 & 0.08849700 \\
\hline $\mathrm{C}$ & -5.48960700 & -2.80283300 & -0.15532700 \\
\hline $\mathrm{C}$ & -4.68065000 & -1.68431400 & 0.07548700 \\
\hline $\mathrm{C}$ & -3.26349400 & -1.42675600 & -0.05148600 \\
\hline $\mathrm{C}$ & -2.16481300 & -2.19554200 & -0.45077800 \\
\hline $\mathrm{C}$ & -0.89591700 & -1.63006800 & -0.44477600 \\
\hline $\mathrm{C}$ & -0.68403200 & -0.28939600 & -0.04329300 \\
\hline $\mathrm{C}$ & -1.77958200 & 0.49067600 & 0.35399200 \\
\hline $\mathrm{C}$ & -3.05388200 & -0.07963500 & 0.34788200 \\
\hline $\mathrm{C}$ & -4.50318000 & 1.83383300 & 1.19982900 \\
\hline $\mathrm{C}$ & -5.04441700 & 2.80929900 & 0.14087200 \\
\hline
\end{tabular}




\begin{tabular}{|c|c|c|c|}
\hline $\mathrm{C}$ & -4.07042300 & 3.11116900 & -1.00441300 \\
\hline $\mathrm{C}$ & -4.65629200 & 4.07656100 & -2.03994400 \\
\hline $\mathrm{C}$ & 3.05388100 & 0.07963400 & 0.34788300 \\
\hline $\mathrm{C}$ & 1.77958200 & -0.49067600 & 0.35399200 \\
\hline $\mathrm{C}$ & 0.68403100 & 0.28939600 & -0.04329300 \\
\hline $\mathrm{C}$ & 0.89591600 & 1.63006800 & -0.44477700 \\
\hline $\mathrm{C}$ & 2.16481200 & 2.19554100 & -0.45077800 \\
\hline $\mathrm{C}$ & 3.26349400 & 1.42675600 & -0.05148600 \\
\hline $\mathrm{C}$ & 4.68064900 & 1.68431400 & 0.07548700 \\
\hline $\mathrm{C}$ & 5.48960600 & 2.80283300 & -0.15532700 \\
\hline $\mathrm{C}$ & 6.85754900 & 2.71371900 & 0.08849800 \\
\hline $\mathrm{C}$ & 7.42337900 & 1.51744600 & 0.56187800 \\
\hline $\mathrm{C}$ & 6.64187000 & 0.38771700 & 0.79771300 \\
\hline $\mathrm{C}$ & 5.26824800 & 0.48102900 & 0.55098900 \\
\hline $\mathrm{C}$ & 4.50317900 & -1.83383400 & 1.19982900 \\
\hline $\mathrm{C}$ & 5.04441900 & -2.80929900 & 0.14087200 \\
\hline $\mathrm{C}$ & 4.07042500 & -3.11116900 & -1.00441300 \\
\hline $\mathrm{C}$ & 4.65629500 & -4.07656000 & -2.03994500 \\
\hline $\mathrm{H}$ & -7.09334500 & 0.53100500 & 1.15979100 \\
\hline $\mathrm{H}$ & -7.49510800 & -3.57540900 & -0.08771100 \\
\hline $\mathrm{H}$ & -5.05437400 & -3.73025600 & -0.51886300 \\
\hline $\mathrm{H}$ & -2.30158900 & -3.22483200 & -0.77269300 \\
\hline $\mathrm{H}$ & -0.04818900 & -2.21605500 & -0.78648300 \\
\hline $\mathrm{H}$ & -1.62140400 & 1.51220400 & 0.68347500 \\
\hline $\mathrm{H}$ & -3.55785700 & 2.20452200 & 1.60949300 \\
\hline $\mathrm{H}$ & -5.20467400 & 1.77363000 & 2.04101700 \\
\hline $\mathrm{H}$ & -5.30878400 & 3.74466200 & 0.65513900 \\
\hline $\mathrm{H}$ & -5.97998900 & 2.40672300 & -0.26983800 \\
\hline $\mathrm{H}$ & -3.14497000 & 3.53662700 & -0.59000800 \\
\hline $\mathrm{H}$ & -3.78413700 & 2.17399400 & -1.49744600 \\
\hline $\mathrm{H}$ & -4.92461300 & 5.03718800 & -1.58293300 \\
\hline $\mathrm{H}$ & -5.56313400 & 3.66274300 & -2.49756400 \\
\hline $\mathrm{H}$ & -3.94051200 & 4.27851000 & -2.84450800 \\
\hline $\mathrm{H}$ & 1.62140300 & -1.51220400 & 0.68347500 \\
\hline $\mathrm{H}$ & 0.04818800 & 2.21605400 & -0.78648300 \\
\hline
\end{tabular}




\begin{tabular}{llll}
\hline $\mathrm{H}$ & 2.30158800 & 3.22483100 & -0.77269300 \\
$\mathrm{H}$ & 5.05437300 & 3.73025600 & -0.51886300 \\
$\mathrm{H}$ & 7.49510700 & 3.57540900 & -0.08771100 \\
$\mathrm{H}$ & 7.09334400 & -0.53100500 & 1.15979300 \\
$\mathrm{H}$ & 3.55785700 & -2.20452300 & 1.60949200 \\
$\mathrm{H}$ & 5.20467200 & -1.77363000 & 2.04101900 \\
$\mathrm{H}$ & 5.97999100 & -2.40672100 & -0.26983700 \\
$\mathrm{H}$ & 5.30878600 & -3.74466100 & 0.65513900 \\
$\mathrm{H}$ & 3.14497200 & -3.53662800 & -0.59000800 \\
$\mathrm{H}$ & 3.78413800 & -2.17399400 & -1.49744500 \\
$\mathrm{H}$ & 4.92461800 & -5.03718700 & -1.58293400 \\
$\mathrm{H}$ & 5.56313700 & -3.66274000 & -2.49756500 \\
$\mathrm{H}$ & 3.94051500 & -4.27851000 & -2.84450900 \\
$\mathrm{H}$ & 8.49317600 & 1.46854900 & 0.74669300 \\
$\mathrm{H}$ & -8.49317700 & -1.46854900 & 0.74669200 \\
$\mathrm{~N}$ & -4.27593100 & 0.48093300 & 0.71281600 \\
$\mathrm{~N}$ & 4.27593100 & -0.48093300 & 0.71281600 \\
\hline $\mathrm{N}$ & $\mathrm{y}$ & &
\end{tabular}

Number of imaginary frequency: 0

Table $S 18$ Atomic coordinates of [3]L-Bu-Cbz after geometry optimization (b3lyp/6-31g(d))

\begin{tabular}{llll}
\hline Atom & $\mathbf{X}(\AA)$ & $\mathbf{Y}(\AA)$ & $\mathbf{Z}(\AA)$ \\
\hline $\mathrm{C}$ & -9.39029200 & -0.19743500 & 0.88864200 \\
$\mathrm{C}$ & -10.69809600 & -0.53668100 & 1.25054900 \\
$\mathrm{C}$ & -11.68254000 & 0.44192200 & 1.12456100 \\
$\mathrm{C}$ & -11.37944400 & 1.72861600 & 0.64720000 \\
$\mathrm{C}$ & -10.07618700 & 2.06274200 & 0.28872100 \\
$\mathrm{C}$ & -9.06724600 & 1.10010300 & 0.40775400 \\
$\mathrm{C}$ & -7.64461700 & 1.09953400 & 0.14923700 \\
$\mathrm{C}$ & -6.73775900 & 2.05861100 & -0.31480800 \\
$\mathrm{C}$ & -5.39455800 & 1.72645700 & -0.43845700 \\
$\mathrm{C}$ & -4.91655400 & 0.43628100 & -0.10609100 \\
$\mathrm{C}$ & -5.81868000 & -0.53335100 & 0.35522800 \\
$\mathrm{C}$ & -7.16797300 & -0.19731600 & 0.47976600 \\
$\mathrm{C}$ & -8.17746400 & -2.35280100 & 1.38669000 \\
$\mathrm{C}$ & -8.63801100 & -3.38867400 & 0.34720800 \\
\hline
\end{tabular}




\begin{tabular}{|c|c|c|c|}
\hline $\mathrm{C}$ & -7.73915800 & -3.49235400 & -0.89086700 \\
\hline $\mathrm{C}$ & -8.24270700 & -4.52752100 & -1.90200200 \\
\hline $\mathrm{C}$ & -1.15197700 & 0.72287600 & -0.05020000 \\
\hline $\mathrm{C}$ & -2.49920500 & 1.06206400 & 0.08542800 \\
\hline $\mathrm{C}$ & -3.47441100 & 0.10893100 & -0.24406000 \\
\hline $\mathrm{C}$ & -3.06893600 & -1.16528600 & -0.70854400 \\
\hline $\mathrm{C}$ & -1.72692200 & -1.50046200 & -0.84078200 \\
\hline $\mathrm{C}$ & -0.74668400 & -0.55830000 & -0.50992800 \\
\hline $\mathrm{C}$ & 0.69771500 & -0.56133500 & -0.50472400 \\
\hline $\mathrm{C}$ & 1.67314000 & -1.50977800 & -0.83229800 \\
\hline $\mathrm{C}$ & 3.01663600 & -1.18394100 & -0.69615900 \\
\hline $\mathrm{C}$ & 3.42736600 & 0.08888100 & -0.23266300 \\
\hline $\mathrm{C}$ & 2.45727800 & 1.04789600 & 0.09360500 \\
\hline $\mathrm{C}$ & 1.10766700 & 0.71876400 & -0.04294000 \\
\hline $\mathrm{C}$ & -0.04710900 & 2.84835400 & 0.74390900 \\
\hline $\mathrm{C}$ & -0.30711500 & 3.92386200 & -0.32436900 \\
\hline $\mathrm{C}$ & 0.80480500 & 4.06441500 & -1.37092800 \\
\hline $\mathrm{C}$ & 0.49695800 & 5.13740300 & -2.42046900 \\
\hline $\mathrm{C}$ & 7.11859600 & -0.23254100 & 0.49684700 \\
\hline $\mathrm{C}$ & 5.76784400 & -0.56258000 & 0.37185400 \\
\hline $\mathrm{C}$ & 4.87091800 & 0.40984000 & -0.09362200 \\
\hline $\mathrm{C}$ & 5.35539300 & 1.69661600 & -0.42986300 \\
\hline $\mathrm{C}$ & 6.70003200 & 2.02274600 & -0.30570900 \\
\hline $\mathrm{C}$ & 7.60177100 & 1.06093300 & 0.16264400 \\
\hline $\mathrm{C}$ & 9.02411300 & 1.05557700 & 0.42271900 \\
\hline $\mathrm{C}$ & 10.03775800 & 2.01300100 & 0.30165500 \\
\hline $\mathrm{C}$ & 11.33904400 & 1.67384000 & 0.66258500 \\
\hline $\mathrm{C}$ & 11.63551100 & 0.38727000 & 1.14440900 \\
\hline $\mathrm{C}$ & 10.64627300 & -0.58621900 & 1.27251100 \\
\hline $\mathrm{C}$ & 9.34047500 & -0.24192800 & 0.90813000 \\
\hline $\mathrm{C}$ & 8.11692500 & -2.39001800 & 1.41133200 \\
\hline $\mathrm{C}$ & 8.57399800 & -3.43100300 & 0.37542100 \\
\hline $\mathrm{C}$ & 7.67645900 & -3.53382800 & -0.86367900 \\
\hline $\mathrm{C}$ & 8.17649300 & -4.57423400 & -1.87117500 \\
\hline $\mathrm{H}$ & -10.94762300 & -1.52772800 & 1.61731600 \\
\hline
\end{tabular}




\begin{tabular}{|c|c|c|c|}
\hline $\mathrm{H}$ & -12.17065300 & 2.46762000 & 0.55836100 \\
\hline $\mathrm{H}$ & -9.84422500 & 3.05963100 & -0.07746300 \\
\hline $\mathrm{H}$ & -7.08046300 & 3.05405800 & -0.58570900 \\
\hline $\mathrm{H}$ & -4.69660000 & 2.46042700 & -0.82944500 \\
\hline $\mathrm{H}$ & -5.45550000 & -1.51728300 & 0.63283900 \\
\hline $\mathrm{H}$ & -8.79673900 & -2.43265100 & 2.28862600 \\
\hline $\mathrm{H}$ & -7.14795500 & -2.55876800 & 1.69737700 \\
\hline $\mathrm{H}$ & -9.66401400 & -3.15050800 & 0.03639700 \\
\hline $\mathrm{H}$ & -8.68539200 & -4.36538800 & 0.85025500 \\
\hline $\mathrm{H}$ & -7.66927600 & -2.51051800 & -1.37510200 \\
\hline $\mathrm{H}$ & -6.71851400 & -3.75435300 & -0.57644000 \\
\hline $\mathrm{H}$ & -9.24707100 & -4.27274200 & -2.26166600 \\
\hline $\mathrm{H}$ & -8.29464100 & -5.52851200 & -1.45593000 \\
\hline $\mathrm{H}$ & -7.58208700 & -4.58503400 & -2.77419500 \\
\hline $\mathrm{H}$ & -2.79911500 & 2.03278500 & 0.46669700 \\
\hline $\mathrm{H}$ & -3.82930300 & -1.88442900 & -0.99751500 \\
\hline $\mathrm{H}$ & -1.44430900 & -2.48425700 & -1.20687900 \\
\hline $\mathrm{H}$ & 1.38446400 & -2.49130100 & -1.19977300 \\
\hline $\mathrm{H}$ & 3.77354700 & -1.90788600 & -0.98211900 \\
\hline $\mathrm{H}$ & 2.76508700 & 2.01772200 & 0.47009500 \\
\hline $\mathrm{H}$ & 0.90855200 & 3.03394900 & 1.24495500 \\
\hline $\mathrm{H}$ & -0.82092200 & 2.90160100 & 1.51947300 \\
\hline $\mathrm{H}$ & -0.44587300 & 4.88244600 & 0.19620700 \\
\hline $\mathrm{H}$ & -1.25850300 & 3.70421600 & -0.82705000 \\
\hline $\mathrm{H}$ & 1.74985400 & 4.30854900 & -0.86446400 \\
\hline $\mathrm{H}$ & 0.96290900 & 3.09959200 & -1.86845700 \\
\hline $\mathrm{H}$ & 0.36374900 & 6.12266300 & -1.95661600 \\
\hline $\mathrm{H}$ & -0.42371000 & 4.90200700 & -2.96817000 \\
\hline $\mathrm{H}$ & 1.30736100 & 5.22064300 & -3.15311600 \\
\hline $\mathrm{H}$ & 5.39965100 & -1.54392600 & 0.65204200 \\
\hline $\mathrm{H}$ & 4.66137400 & 2.43233000 & -0.82462600 \\
\hline $\mathrm{H}$ & 7.04787500 & 3.01557300 & -0.57969700 \\
\hline $\mathrm{H}$ & 9.81093300 & 3.00979500 & -0.06799500 \\
\hline $\mathrm{H}$ & 12.13385600 & 2.40878100 & 0.57218800 \\
\hline $\mathrm{H}$ & 10.89069600 & -1.57725400 & 1.64273300 \\
\hline
\end{tabular}




\begin{tabular}{llll}
\hline $\mathrm{H}$ & 7.08606500 & -2.59029700 & 1.72124600 \\
$\mathrm{H}$ & 8.73465600 & -2.47016700 & 2.31430500 \\
$\mathrm{H}$ & 9.60157900 & -3.19862900 & 0.06543800 \\
$\mathrm{H}$ & 8.61596900 & -4.40652100 & 0.88126300 \\
$\mathrm{H}$ & 6.65410200 & -3.79000900 & -0.55003700 \\
$\mathrm{H}$ & 7.61202700 & -2.55302900 & -1.35076000 \\
$\mathrm{H}$ & 8.22296800 & -5.57421200 & -1.42223400 \\
$\mathrm{H}$ & 9.18259300 & -4.32529300 & -2.23008000 \\
$\mathrm{H}$ & 7.51687100 & -4.63101400 & -2.74417100 \\
$\mathrm{H}$ & -12.70589100 & 0.20125700 & 1.39995800 \\
$\mathrm{H}$ & 12.65741700 & 0.14262000 & 1.42165700 \\
$\mathrm{~N}$ & -8.23514200 & -0.97226300 & 0.92856300 \\
$\mathrm{~N}$ & -0.02259500 & 1.48533700 & 0.23280400 \\
$\mathrm{~N}$ & 8.18160500 & -1.01110600 & 0.94929700 \\
\hline
\end{tabular}

Number of imaginary frequency: 0

Table S 19 Atomic coordinates of [4]C-Hex-Cbz after geometry optimization (b3lyp/6-31g(d))

\begin{tabular}{llll}
\hline Atom & $\mathbf{X}(\AA)$ & $\mathbf{Y}(\AA)$ & $\mathbf{Z}(\AA)$ \\
\hline $\mathrm{C}$ & -4.76932400 & 0.57522000 & -1.24132600 \\
$\mathrm{C}$ & -4.11053500 & 2.69999400 & -1.58060400 \\
$\mathrm{C}$ & -3.58303900 & 1.08084000 & -3.43624100 \\
$\mathrm{C}$ & 2.69632000 & 4.77125900 & -0.02401100 \\
$\mathrm{C}$ & 0.54556000 & 5.41240300 & -0.19505800 \\
$\mathrm{C}$ & -0.20280400 & -4.39764400 & 1.46449100 \\
$\mathrm{C}$ & -2.35332800 & -3.75814400 & 1.28489400 \\
$\mathrm{C}$ & -3.24770100 & 3.73571900 & -1.94351600 \\
$\mathrm{C}$ & -4.77923800 & 2.68584800 & -0.32814500 \\
$\mathrm{C}$ & -2.99724800 & 4.76327900 & -1.02265000 \\
$\mathrm{C}$ & -3.78827900 & 4.83760100 & 0.15015000 \\
$\mathrm{C}$ & -1.67220300 & 5.44149800 & -1.14197000 \\
$\mathrm{C}$ & -0.83789600 & 5.35842300 & -0.01822000 \\
$\mathrm{C}$ & -1.10534200 & 5.79218600 & -2.39170600 \\
$\mathrm{C}$ & 0.27320800 & 5.87851700 & -2.56062600 \\
$\mathrm{C}$ & 1.12479300 & 5.60198400 & -1.47900700 \\
$\mathrm{C}$ & 2.51015200 & 5.18597800 & -1.36920100
\end{tabular}




\begin{tabular}{|c|c|c|c|}
\hline $\mathrm{C}$ & -4.67235300 & 3.81872200 & 0.49425400 \\
\hline $\mathrm{C}$ & -5.20707400 & 1.31680100 & -0.11062600 \\
\hline $\mathrm{C}$ & -5.68591600 & 0.61165400 & 1.00512300 \\
\hline $\mathrm{C}$ & -5.56167200 & -0.77369600 & 1.05024400 \\
\hline $\mathrm{C}$ & -4.96817800 & -1.49319900 & -0.01551500 \\
\hline $\mathrm{C}$ & -4.67008300 & -0.81693100 & -1.20719600 \\
\hline $\mathrm{C}$ & -4.27157300 & -2.79849200 & 0.18596000 \\
\hline $\mathrm{C}$ & -3.44361200 & -2.88678600 & 1.31445400 \\
\hline $\mathrm{C}$ & -4.11027200 & -3.74457700 & -0.85575300 \\
\hline $\mathrm{C}$ & -2.09156700 & -4.59316200 & 0.16671400 \\
\hline $\mathrm{C}$ & -3.04049300 & -4.63546800 & -0.86701800 \\
\hline $\mathrm{C}$ & -0.70630000 & -5.00753400 & 0.28363500 \\
\hline $\mathrm{C}$ & -4.29584800 & 1.65311400 & -4.67399200 \\
\hline $\mathrm{C}$ & -5.74406500 & 1.17875400 & -4.87128200 \\
\hline $\mathrm{C}$ & -5.89847700 & -0.31815400 & -5.17523800 \\
\hline $\mathrm{C}$ & -7.34952400 & -0.72661200 & -5.46249400 \\
\hline $\mathrm{C}$ & -7.50927500 & -2.22092100 & -5.75969600 \\
\hline $\mathrm{C}$ & 2.01009700 & 5.64529800 & 3.07084500 \\
\hline $\mathrm{C}$ & 1.34506300 & 4.65745400 & 2.09867700 \\
\hline $\mathrm{C}$ & 1.89903500 & 5.23338100 & 4.54807600 \\
\hline $\mathrm{C}$ & 0.47039400 & 5.25753000 & 5.11408300 \\
\hline $\mathrm{C}$ & 0.37100600 & 4.87780100 & 6.60189200 \\
\hline $\mathrm{C}$ & 1.03029400 & 5.87643300 & 7.56087200 \\
\hline $\mathrm{C}$ & 3.77661700 & 3.97450900 & 0.35884800 \\
\hline $\mathrm{C}$ & 3.52072500 & 4.89458100 & -2.29934700 \\
\hline $\mathrm{C}$ & 4.67113800 & 3.52730900 & -0.62424400 \\
\hline $\mathrm{C}$ & 4.58055000 & 4.07138500 & -1.92901700 \\
\hline $\mathrm{C}$ & 5.36759300 & 2.23804800 & -0.33781800 \\
\hline $\mathrm{C}$ & 5.14478000 & 1.20103900 & -1.25522200 \\
\hline $\mathrm{C}$ & 5.88234100 & 1.91861800 & 0.94271600 \\
\hline $\mathrm{C}$ & 5.22839100 & -0.12049700 & -0.81316900 \\
\hline $\mathrm{C}$ & 5.99505500 & 0.59859400 & 1.37023700 \\
\hline $\mathrm{C}$ & 5.58181200 & -0.44254200 & 0.52376000 \\
\hline $\mathrm{C}$ & 4.54806600 & -2.23844600 & -0.47371400 \\
\hline $\mathrm{C}$ & 4.18549800 & -1.26678800 & -2.79874500 \\
\hline
\end{tabular}




\begin{tabular}{|c|c|c|c|}
\hline $\mathrm{C}$ & 5.14617300 & -1.80899800 & 0.74198400 \\
\hline $\mathrm{C}$ & 3.69457400 & -3.34249700 & -0.51716900 \\
\hline $\mathrm{C}$ & 5.27496700 & -1.24092200 & -3.88514900 \\
\hline $\mathrm{C}$ & 4.98327900 & -2.60857500 & 1.88457900 \\
\hline $\mathrm{C}$ & 3.38777500 & -4.01481400 & 0.67446500 \\
\hline $\mathrm{C}$ & 6.23317100 & -2.44212300 & -3.87802000 \\
\hline $\mathrm{C}$ & 4.11143100 & -3.69266500 & 1.84849700 \\
\hline $\mathrm{C}$ & 2.06651900 & -4.70997500 & 0.71019500 \\
\hline $\mathrm{C}$ & 5.58350700 & -3.78981100 & -4.22244500 \\
\hline $\mathrm{C}$ & 1.16830300 & -4.27039300 & 1.69292300 \\
\hline $\mathrm{C}$ & 1.57183600 & -5.46181700 & -0.38339600 \\
\hline $\mathrm{C}$ & 6.59466900 & -4.94136000 & -4.30247300 \\
\hline $\mathrm{C}$ & 0.20505400 & -5.61624200 & -0.59401300 \\
\hline $\mathrm{C}$ & 5.94996100 & -6.28955900 & -4.63937600 \\
\hline $\mathrm{C}$ & -1.13210900 & -2.92106200 & 3.32000100 \\
\hline $\mathrm{C}$ & -1.90346300 & -3.48042900 & 4.52675400 \\
\hline $\mathrm{C}$ & -1.35709100 & -4.82281400 & 5.02957500 \\
\hline $\mathrm{C}$ & -1.99820100 & -5.37135600 & 6.31758600 \\
\hline $\mathrm{C}$ & -3.49322600 & -5.75188100 & 6.23015000 \\
\hline $\mathrm{C}$ & -4.47750300 & -4.62725100 & 6.57971100 \\
\hline $\mathrm{H}$ & -2.61978100 & 3.64393900 & -2.82326400 \\
\hline $\mathrm{H}$ & -1.75790000 & 5.91477700 & -3.25194600 \\
\hline $\mathrm{H}$ & 0.68487000 & 6.07254800 & -3.54801400 \\
\hline $\mathrm{H}$ & -1.26179300 & 5.04655500 & 0.93010100 \\
\hline $\mathrm{H}$ & -3.63884100 & 5.66789300 & 0.83517700 \\
\hline $\mathrm{H}$ & -5.20551400 & 3.86425300 & 1.44068400 \\
\hline $\mathrm{H}$ & -6.07038900 & 1.14733500 & 1.86958800 \\
\hline $\mathrm{H}$ & -5.84736500 & -1.30671900 & 1.95308500 \\
\hline $\mathrm{H}$ & -3.53029000 & -2.14193800 & 2.09824100 \\
\hline $\mathrm{H}$ & -2.89707300 & -5.29500000 & -1.71935300 \\
\hline $\mathrm{H}$ & -4.78986700 & -3.71657500 & -1.70331400 \\
\hline $\mathrm{H}$ & -4.16816700 & -1.35362400 & -2.00476200 \\
\hline $\mathrm{H}$ & -3.54988800 & -0.00914600 & -3.48814200 \\
\hline $\mathrm{H}$ & -2.53990500 & 1.42444200 & -3.41825000 \\
\hline $\mathrm{H}$ & -4.28277800 & 2.74840700 & -4.60983800 \\
\hline
\end{tabular}




\begin{tabular}{|c|c|c|c|}
\hline $\mathrm{H}$ & -3.69394100 & 1.38695200 & -5.55464900 \\
\hline $\mathrm{H}$ & -6.18140600 & 1.75422000 & -5.69888900 \\
\hline $\mathrm{H}$ & -6.33268400 & 1.43408400 & -3.97997400 \\
\hline $\mathrm{H}$ & -5.26957000 & -0.58279000 & -6.03918300 \\
\hline $\mathrm{H}$ & -5.52693300 & -0.91542300 & -4.33062900 \\
\hline $\mathrm{H}$ & -7.97687700 & -0.45512200 & -4.60201900 \\
\hline $\mathrm{H}$ & -7.72815600 & -0.14092600 & -6.31228600 \\
\hline $\mathrm{H}$ & -6.91754400 & -2.51746100 & -6.63473000 \\
\hline $\mathrm{H}$ & -8.55494700 & -2.47882600 & -5.96243400 \\
\hline $\mathrm{H}$ & -7.17488100 & -2.83159700 & -4.91191100 \\
\hline $\mathrm{H}$ & 0.27074700 & 4.60077300 & 2.28405600 \\
\hline $\mathrm{H}$ & 1.74335200 & 3.64572400 & 2.25837500 \\
\hline $\mathrm{H}$ & 1.56536700 & 6.63834100 & 2.92235100 \\
\hline $\mathrm{H}$ & 3.06908300 & 5.73767200 & 2.79928600 \\
\hline $\mathrm{H}$ & 2.53503700 & 5.90696400 & 5.13551600 \\
\hline $\mathrm{H}$ & 2.32327700 & 4.22606000 & 4.67907500 \\
\hline $\mathrm{H}$ & 0.04197700 & 6.26062100 & 4.96773600 \\
\hline $\mathrm{H}$ & -0.16467000 & 4.56858200 & 4.54075700 \\
\hline $\mathrm{H}$ & -0.69047300 & 4.78102000 & 6.86632500 \\
\hline $\mathrm{H}$ & 0.81202500 & 3.88150800 & 6.75009800 \\
\hline $\mathrm{H}$ & 0.60586800 & 6.88068000 & 7.43686500 \\
\hline $\mathrm{H}$ & 0.87642100 & 5.57778700 & 8.60404100 \\
\hline $\mathrm{H}$ & 2.11134900 & 5.95107300 & 7.39874400 \\
\hline $\mathrm{H}$ & 3.80976400 & 3.53377800 & 1.34951000 \\
\hline $\mathrm{H}$ & 3.43518200 & 5.23286900 & -3.32904700 \\
\hline $\mathrm{H}$ & 5.31014800 & 3.77247700 & -2.67686600 \\
\hline $\mathrm{H}$ & 4.70993800 & 1.42935400 & -2.22240800 \\
\hline $\mathrm{H}$ & 6.11446700 & 2.72454500 & 1.63376400 \\
\hline $\mathrm{H}$ & 6.31595300 & 0.38678800 & 2.38720600 \\
\hline $\mathrm{H}$ & 3.56578400 & -2.15937100 & -2.90422000 \\
\hline $\mathrm{H}$ & 3.51607400 & -0.40524200 & -2.92565200 \\
\hline $\mathrm{H}$ & 3.10656400 & -3.55047400 & -1.40437000 \\
\hline $\mathrm{H}$ & 5.85691100 & -0.31728700 & -3.77460500 \\
\hline $\mathrm{H}$ & 4.76929100 & -1.17404500 & -4.85906400 \\
\hline $\mathrm{H}$ & 5.46429000 & -2.33383100 & 2.82011600 \\
\hline
\end{tabular}




\begin{tabular}{|c|c|c|c|}
\hline $\mathrm{H}$ & 7.03590000 & -2.24191800 & -4.60115200 \\
\hline $\mathrm{H}$ & 6.71977800 & -2.51050500 & -2.89585800 \\
\hline $\mathrm{H}$ & 3.91691700 & -4.25268000 & 2.75923000 \\
\hline $\mathrm{H}$ & 5.05343600 & -3.70534000 & -5.18361200 \\
\hline $\mathrm{H}$ & 4.82111700 & -4.04425300 & -3.47270500 \\
\hline $\mathrm{H}$ & 1.53802400 & -3.65672600 & 2.50704000 \\
\hline $\mathrm{H}$ & 2.27314800 & -5.85387800 & -1.11508700 \\
\hline $\mathrm{H}$ & 7.12780300 & -5.01936900 & -3.34467300 \\
\hline $\mathrm{H}$ & 7.35728000 & -4.70056500 & -5.05677600 \\
\hline $\mathrm{H}$ & -0.14938500 & -6.13056000 & -1.48388900 \\
\hline $\mathrm{H}$ & 5.43456000 & -6.25265300 & -5.60724200 \\
\hline $\mathrm{H}$ & 6.69802400 & -7.08878600 & -4.69120200 \\
\hline $\mathrm{H}$ & 5.21023100 & -6.57556400 & -3.88143400 \\
\hline $\mathrm{H}$ & -0.07127600 & -2.83237000 & 3.56868300 \\
\hline $\mathrm{H}$ & -1.48612400 & -1.90892600 & 3.08236200 \\
\hline $\mathrm{H}$ & -0.27642300 & -4.71460600 & 5.20520100 \\
\hline $\mathrm{H}$ & -2.96056800 & -3.57706400 & 4.25343500 \\
\hline $\mathrm{H}$ & -1.85404700 & -2.73167400 & 5.33026300 \\
\hline $\mathrm{H}$ & -1.45985100 & -5.57168500 & 4.23298900 \\
\hline $\mathrm{H}$ & -1.42744700 & -6.26491300 & 6.60264400 \\
\hline $\mathrm{H}$ & -1.85328700 & -4.65122500 & 7.13683800 \\
\hline $\mathrm{H}$ & -3.68067100 & -6.58803800 & 6.91654800 \\
\hline $\mathrm{H}$ & -3.71207600 & -6.13825200 & 5.22452700 \\
\hline $\mathrm{H}$ & -4.39055900 & -3.76989100 & 5.90443300 \\
\hline $\mathrm{H}$ & -5.51307000 & -4.98338000 & 6.52784600 \\
\hline $\mathrm{H}$ & -4.30407100 & -4.26127500 & 7.59942500 \\
\hline $\mathrm{N}$ & -4.22193000 & 1.44828800 & -2.18103300 \\
\hline $\mathrm{N}$ & 1.53736400 & 5.02457200 & 0.70461900 \\
\hline $\mathrm{N}$ & -1.24431000 & -3.74811200 & 2.12721600 \\
\hline $\mathrm{N}$ & 4.72047000 & -1.25279900 & -1.44514000 \\
\hline
\end{tabular}

Number of imaginary frequency: 0 
Table S 20 Atomic coordinates of [2]L-Hex-Cbz after geometry optimization (b3lyp/6-31g(d))

\begin{tabular}{|c|c|c|c|}
\hline Atom & $\mathbf{X}(\AA)$ & $Y(\AA)$ & $Z(\AA)$ \\
\hline $\mathrm{C}$ & 2.65110700 & -0.38208800 & 0.66558000 \\
\hline $\mathrm{C}$ & 4.78434600 & -1.06635000 & 0.96020700 \\
\hline $\mathrm{C}$ & 6.79071700 & -2.36586600 & 1.03286700 \\
\hline $\mathrm{C}$ & 6.14315200 & -1.15656800 & 1.27977000 \\
\hline $\mathrm{C}$ & 6.10830600 & -3.46226200 & 0.47834200 \\
\hline $\mathrm{C}$ & 4.07960600 & -2.16711300 & 0.40264400 \\
\hline $\mathrm{C}$ & 4.75541000 & -3.36895800 & 0.16265700 \\
\hline $\mathrm{C}$ & 2.71425400 & -1.72876900 & 0.21736900 \\
\hline $\mathrm{C}$ & -7.53099600 & 2.54007600 & 0.49874700 \\
\hline $\mathrm{C}$ & -6.91282100 & 1.31101200 & 0.72231300 \\
\hline $\mathrm{C}$ & -6.79678600 & 3.66485300 & 0.08523000 \\
\hline $\mathrm{C}$ & -5.53043300 & 1.22946600 & 0.52417500 \\
\hline $\mathrm{C}$ & -5.42054200 & 3.58056800 & -0.10817300 \\
\hline $\mathrm{C}$ & -4.77374700 & 2.35912900 & 0.11197100 \\
\hline $\mathrm{C}$ & -3.37982100 & 0.54903900 & 0.39068300 \\
\hline $\mathrm{C}$ & -5.10508000 & -1.18328900 & 1.11876200 \\
\hline $\mathrm{C}$ & -3.39777500 & 1.92325300 & 0.02979900 \\
\hline $\mathrm{C}$ & -2.18825500 & -0.17798700 & 0.43208600 \\
\hline $\mathrm{C}$ & -5.78041200 & -2.01769000 & 0.01401100 \\
\hline $\mathrm{C}$ & -2.19407900 & 2.55670200 & -0.29734500 \\
\hline $\mathrm{C}$ & -0.98593500 & 0.46753000 & 0.10823200 \\
\hline $\mathrm{C}$ & -4.85416500 & -2.47558800 & -1.12454500 \\
\hline $\mathrm{C}$ & -1.00803600 & 1.83461400 & -0.25700200 \\
\hline $\mathrm{C}$ & 0.29800000 & -0.27857200 & 0.15007200 \\
\hline $\mathrm{C}$ & -3.88692900 & -3.60414900 & -0.74012500 \\
\hline $\mathrm{C}$ & 1.45809500 & 0.34256800 & 0.63456300 \\
\hline $\mathrm{C}$ & 0.36452700 & -1.61876600 & -0.30005600 \\
\hline $\mathrm{C}$ & -2.98027100 & -4.04489100 & -1.89676600 \\
\hline $\mathrm{C}$ & 1.55269800 & -2.33802100 & -0.26957900 \\
\hline $\mathrm{C}$ & -2.01768000 & -5.17371800 & -1.51402400 \\
\hline $\mathrm{C}$ & 4.27842000 & 1.29979800 & 1.67302000 \\
\hline C & 5.00418800 & 2.23516400 & 0.68970500 \\
\hline $\mathrm{C}$ & 4.13368300 & 2.70249900 & -0.48385400 \\
\hline
\end{tabular}




\begin{tabular}{|c|c|c|c|}
\hline $\mathrm{C}$ & 4.77966100 & 3.73302400 & -1.42853000 \\
\hline $\mathrm{C}$ & 6.01161900 & 3.25000900 & -2.22685900 \\
\hline $\mathrm{C}$ & 7.36715500 & 3.49737800 & -1.55081900 \\
\hline $\mathrm{H}$ & 6.68407000 & -0.31696900 & 1.70572000 \\
\hline $\mathrm{H}$ & 4.22941400 & -4.21966200 & -0.26317000 \\
\hline $\mathrm{H}$ & 6.64307000 & -4.39002100 & 0.29578000 \\
\hline $\mathrm{H}$ & -7.49349200 & 0.45052600 & 1.04035700 \\
\hline $\mathrm{H}$ & -7.30948300 & 4.60764400 & -0.08307900 \\
\hline $\mathrm{H}$ & -4.85432700 & 4.45325200 & -0.42365600 \\
\hline $\mathrm{H}$ & -4.23052100 & -1.70120000 & 1.52235600 \\
\hline $\mathrm{H}$ & -5.80179100 & -1.05417700 & 1.95642100 \\
\hline $\mathrm{H}$ & -2.17292200 & -1.21843500 & 0.73897100 \\
\hline $\mathrm{H}$ & -6.60521200 & -1.42751900 & -0.40352600 \\
\hline $\mathrm{H}$ & -6.23851300 & -2.89618100 & 0.49090800 \\
\hline $\mathrm{H}$ & -2.18541700 & 3.60407800 & -0.58840400 \\
\hline $\mathrm{H}$ & -5.47723200 & -2.81973500 & -1.96137000 \\
\hline $\mathrm{H}$ & -4.28825000 & -1.61423600 & -1.50447800 \\
\hline $\mathrm{H}$ & -0.07794400 & 2.31714300 & -0.54144200 \\
\hline $\mathrm{H}$ & -4.46514200 & -4.46949100 & -0.38241500 \\
\hline $\mathrm{H}$ & -3.25629800 & -3.29395400 & 0.10571800 \\
\hline $\mathrm{H}$ & 1.40871300 & 1.36259600 & 1.00083800 \\
\hline $\mathrm{H}$ & -0.52885500 & -2.08032200 & -0.70953200 \\
\hline $\mathrm{H}$ & -2.40681300 & -3.17865900 & -2.25621900 \\
\hline $\mathrm{H}$ & -3.60474900 & -4.36587200 & -2.74253000 \\
\hline $\mathrm{H}$ & 1.57883600 & -3.36300100 & -0.63089700 \\
\hline $\mathrm{H}$ & -2.56340600 & -6.06504500 & -1.18052200 \\
\hline $\mathrm{H}$ & -1.38806900 & -5.46684100 & -2.36177800 \\
\hline $\mathrm{H}$ & -1.35381300 & -4.86860400 & -0.69531000 \\
\hline $\mathrm{H}$ & 3.36454100 & 1.77471200 & 2.04459000 \\
\hline $\mathrm{H}$ & 4.91413900 & 1.11832600 & 2.54847600 \\
\hline $\mathrm{H}$ & 3.21058800 & 3.14346200 & -0.07925600 \\
\hline $\mathrm{H}$ & 5.89863900 & 1.72230700 & 0.31783600 \\
\hline $\mathrm{H}$ & 5.35621400 & 3.10675000 & 1.26032400 \\
\hline $\mathrm{H}$ & 3.82027100 & 1.82912500 & -1.07098900 \\
\hline $\mathrm{H}$ & 4.00335100 & 4.04541100 & -2.13920400 \\
\hline
\end{tabular}




\begin{tabular}{llll}
\hline $\mathrm{H}$ & 5.04521600 & 4.63710100 & -0.86041400 \\
$\mathrm{H}$ & 6.02147900 & 3.76247100 & -3.19772600 \\
$\mathrm{H}$ & 5.90025300 & 2.18081400 & -2.45660100 \\
$\mathrm{H}$ & 7.45713400 & 2.97764900 & -0.59137200 \\
$\mathrm{H}$ & 8.19100200 & 3.15630600 & -2.18868600 \\
$\mathrm{H}$ & 7.51734200 & 4.56732200 & -1.35994800 \\
$\mathrm{H}$ & -8.60411900 & 2.62694600 & 0.64662500 \\
$\mathrm{H}$ & 7.84638400 & -2.45928800 & 1.27336800 \\
$\mathrm{~N}$ & 3.91138300 & 0.00615300 & 1.11489500 \\
$\mathrm{~N}$ & -4.67872400 & 0.14085400 & 0.68703100 \\
\hline
\end{tabular}

Number of imaginary frequency: 0

Table S 21 Atomic coordinates of [3]L-Hex-Cbz after geometry optimization (b3lyp/6-31g(d))

\begin{tabular}{llll}
\hline Atom & $\mathbf{X}(\mathbf{\AA})$ & $\mathbf{Y}(\mathbf{\AA})$ & $\mathbf{Z}(\mathbf{\AA})$ \\
\hline $\mathrm{C}$ & 7.34412400 & 0.51236400 & 0.17374100 \\
$\mathrm{C}$ & 9.57994500 & 0.59685300 & 0.48484300 \\
$\mathrm{C}$ & -6.89177900 & -0.32806100 & 0.78236200 \\
$\mathrm{C}$ & -9.08451900 & -0.49222300 & 1.30280700 \\
$\mathrm{C}$ & 11.85207900 & 1.32741200 & 0.63170800 \\
$\mathrm{C}$ & 10.92043600 & 0.29738300 & 0.74760400 \\
$\mathrm{C}$ & 11.46573400 & 2.62654600 & 0.25947200 \\
$\mathrm{C}$ & 10.13088600 & 2.91980900 & -0.00616000 \\
$\mathrm{C}$ & 9.17374500 & 1.90438900 & 0.10233700 \\
$\mathrm{C}$ & 7.74196500 & 1.84910000 & -0.09434900 \\
$\mathrm{C}$ & -11.39672200 & -0.01567700 & 1.68499000 \\
$\mathrm{C}$ & -10.34673600 & -0.93181400 & 1.71533300 \\
$\mathrm{C}$ & -11.20216300 & 1.30732500 & 1.25247100 \\
$\mathrm{C}$ & -8.87097700 & 0.84319000 & 0.86658700 \\
$\mathrm{C}$ & -9.94393600 & 1.74151600 & 0.84377600 \\
$\mathrm{C}$ & -7.46709100 & 0.94751300 & 0.53670800 \\
$\mathrm{C}$ & 8.64527400 & -2.53527100 & -0.41208900 \\
$\mathrm{C}$ & 8.47477600 & -1.65331700 & 0.83642400 \\
$\mathrm{C}$ & 8.59223600 & -4.04429600 & -0.12094200 \\
$\mathrm{C}$ & 9.77560600 & -4.57977300 & 0.70080900 \\
$\mathrm{C}$ & 9.73177600 & -6.09534500 & 0.96305200 \\
\hline & & &
\end{tabular}




\begin{tabular}{|c|c|c|c|}
\hline $\mathrm{C}$ & 9.88964800 & -6.96465500 & -0.29032300 \\
\hline $\mathrm{C}$ & 6.01134400 & 0.10917000 & 0.07669600 \\
\hline $\mathrm{C}$ & 6.76827400 & 2.78421400 & -0.46149200 \\
\hline $\mathrm{C}$ & 5.04241600 & 1.05465300 & -0.29027500 \\
\hline $\mathrm{C}$ & 5.44014000 & 2.38684800 & -0.55657700 \\
\hline $\mathrm{C}$ & 3.61476800 & 0.65945100 & -0.39699400 \\
\hline $\mathrm{C}$ & 2.60546900 & 1.53806900 & 0.02438600 \\
\hline $\mathrm{C}$ & 3.25720900 & -0.60534400 & -0.92245400 \\
\hline $\mathrm{C}$ & 1.27284700 & 1.13635800 & -0.08350300 \\
\hline $\mathrm{C}$ & 1.93010600 & -1.00418000 & -1.02451600 \\
\hline $\mathrm{C}$ & 0.91670800 & -0.13672400 & -0.60202100 \\
\hline $\mathrm{C}$ & -0.98105900 & 1.00725400 & 0.01347300 \\
\hline $\mathrm{C}$ & 0.09814600 & 3.15288400 & 0.87279800 \\
\hline $\mathrm{C}$ & -0.52370000 & -0.21998700 & -0.53925700 \\
\hline $\mathrm{C}$ & -2.33936600 & 1.24769700 & 0.22898800 \\
\hline $\mathrm{C}$ & 0.31847700 & 4.28975000 & -0.14285900 \\
\hline $\mathrm{C}$ & -1.45899700 & -1.20347400 & -0.87969600 \\
\hline $\mathrm{C}$ & -3.26876600 & 0.25312300 & -0.10992000 \\
\hline $\mathrm{C}$ & -0.82369600 & 4.50383700 & -1.14952200 \\
\hline $\mathrm{C}$ & -2.81066800 & -0.96509900 & -0.66580400 \\
\hline $\mathrm{C}$ & -4.71965700 & 0.47841600 & 0.11513900 \\
\hline $\mathrm{C}$ & -2.07772900 & 5.16082100 & -0.55550600 \\
\hline $\mathrm{C}$ & -5.53209900 & -0.56800400 & 0.57500800 \\
\hline $\mathrm{C}$ & -5.29619200 & 1.74693000 & -0.13344500 \\
\hline $\mathrm{C}$ & -3.19754800 & 5.36818900 & -1.58362900 \\
\hline $\mathrm{C}$ & -6.64959200 & 1.98354200 & 0.07207700 \\
\hline $\mathrm{C}$ & -4.44716000 & 6.02805100 & -0.99166800 \\
\hline $\mathrm{C}$ & -7.71435400 & -2.58379700 & 1.63497300 \\
\hline $\mathrm{C}$ & -8.17421800 & -3.59920400 & 0.57383300 \\
\hline $\mathrm{C}$ & -7.33001900 & -3.58756900 & -0.70707600 \\
\hline $\mathrm{C}$ & -7.67925300 & -4.66565500 & -1.74983800 \\
\hline $\mathrm{C}$ & -9.08151100 & -4.56638700 & -2.39197000 \\
\hline $\mathrm{C}$ & -10.18504000 & -5.35874800 & -1.67782600 \\
\hline $\mathrm{H}$ & 12.21727300 & 3.40656500 & 0.17709100 \\
\hline $\mathrm{H}$ & 9.83507000 & 3.92488000 & -0.29606500 \\
\hline
\end{tabular}




\begin{tabular}{|c|c|c|c|}
\hline $\mathrm{H}$ & 11.23530200 & -0.70316100 & 1.02878200 \\
\hline $\mathrm{H}$ & -10.51256500 & -1.95162500 & 2.04914800 \\
\hline $\mathrm{H}$ & -9.79575100 & 2.76631800 & 0.51284400 \\
\hline $\mathrm{H}$ & -12.04230300 & 1.99579900 & 1.23832400 \\
\hline $\mathrm{H}$ & 9.27698200 & -1.83412300 & 1.55848700 \\
\hline $\mathrm{H}$ & 7.53669600 & -1.90095300 & 1.34742200 \\
\hline $\mathrm{H}$ & 9.59606000 & -2.28134900 & -0.89955700 \\
\hline $\mathrm{H}$ & 7.85335800 & -2.27350400 & -1.12504800 \\
\hline $\mathrm{H}$ & 8.54722000 & -4.57290000 & -1.08109400 \\
\hline $\mathrm{H}$ & 7.65220200 & -4.28294000 & 0.39989100 \\
\hline $\mathrm{H}$ & 10.71468700 & -4.32973700 & 0.18411100 \\
\hline $\mathrm{H}$ & 9.81433100 & -4.06443600 & 1.67008100 \\
\hline $\mathrm{H}$ & 10.53038600 & -6.34899500 & 1.67288100 \\
\hline $\mathrm{H}$ & 8.78759000 & -6.34688700 & 1.46717800 \\
\hline $\mathrm{H}$ & 10.82741800 & -6.73662500 & -0.81249700 \\
\hline $\mathrm{H}$ & 9.90563500 & -8.02890200 & -0.02925800 \\
\hline $\mathrm{H}$ & 9.06947400 & -6.81523000 & -1.00132400 \\
\hline $\mathrm{H}$ & 5.71336400 & -0.90872700 & 0.30705900 \\
\hline $\mathrm{H}$ & 7.04675300 & 3.81153200 & -0.68244200 \\
\hline $\mathrm{H}$ & 4.69022200 & 3.10452900 & -0.87487100 \\
\hline $\mathrm{H}$ & 2.87031700 & 2.49831600 & 0.45471900 \\
\hline $\mathrm{H}$ & 4.04140500 & -1.26472900 & -1.28164300 \\
\hline $\mathrm{H}$ & 1.68415200 & -1.97942400 & -1.43702400 \\
\hline $\mathrm{H}$ & -0.85443900 & 3.27343400 & 1.39624400 \\
\hline $\mathrm{H}$ & 0.88034100 & 3.19239000 & 1.64099800 \\
\hline $\mathrm{H}$ & -2.68354100 & 2.17226800 & 0.68021600 \\
\hline $\mathrm{H}$ & 1.24893200 & 4.08573500 & -0.68654200 \\
\hline $\mathrm{H}$ & 0.48790800 & 5.21662300 & 0.42378700 \\
\hline $\mathrm{H}$ & -1.13324400 & -2.14461900 & -1.31547500 \\
\hline $\mathrm{H}$ & -0.45183900 & 5.13703000 & -1.96665400 \\
\hline $\mathrm{H}$ & -1.09020200 & 3.54299700 & -1.61029100 \\
\hline $\mathrm{H}$ & -3.53900700 & -1.71490300 & -0.95928200 \\
\hline $\mathrm{H}$ & -1.80344100 & 6.13218000 & -0.11701500 \\
\hline $\mathrm{H}$ & -2.46780900 & 4.55559000 & 0.27572800 \\
\hline $\mathrm{H}$ & -5.09209400 & -1.53662300 & 0.78771600 \\
\hline
\end{tabular}




\begin{tabular}{|c|c|c|c|}
\hline $\mathrm{H}$ & -4.66879700 & 2.54134600 & -0.52602500 \\
\hline $\mathrm{H}$ & -3.46766500 & 4.39784300 & -2.02396700 \\
\hline $\mathrm{H}$ & -2.81684700 & 5.98086600 & -2.41308900 \\
\hline $\mathrm{H}$ & -7.06923200 & 2.96449000 & -0.13634900 \\
\hline $\mathrm{H}$ & -4.21442700 & 7.01468800 & -0.57228800 \\
\hline $\mathrm{H}$ & -5.22517100 & 6.16447000 & -1.75134900 \\
\hline $\mathrm{H}$ & -4.87267500 & 5.41910900 & -0.18404000 \\
\hline $\mathrm{H}$ & -6.65764800 & -2.73866000 & 1.87618100 \\
\hline $\mathrm{H}$ & -8.27346100 & -2.74239500 & 2.56544400 \\
\hline $\mathrm{H}$ & -6.27299800 & -3.71280900 & -0.42906000 \\
\hline $\mathrm{H}$ & -9.22600300 & -3.40194000 & 0.33678300 \\
\hline $\mathrm{H}$ & -8.13761300 & -4.59775700 & 1.03290200 \\
\hline $\mathrm{H}$ & -7.40404800 & -2.59891900 & -1.17910100 \\
\hline $\mathrm{H}$ & -6.92526300 & -4.59693800 & -2.54487400 \\
\hline $\mathrm{H}$ & -7.55249000 & -5.66357800 & -1.30404500 \\
\hline $\mathrm{H}$ & -9.01933000 & -4.93246800 & -3.42512500 \\
\hline $\mathrm{H}$ & -9.37292800 & -3.50924200 & -2.46862800 \\
\hline $\mathrm{H}$ & -10.34286400 & -5.02091500 & -0.64842100 \\
\hline $\mathrm{H}$ & -11.14081200 & -5.26283500 & -2.20623900 \\
\hline $\mathrm{H}$ & -9.93425200 & -6.42613300 & -1.63822700 \\
\hline $\mathrm{H}$ & -12.38630700 & -0.33468300 & 2.00118100 \\
\hline $\mathrm{H}$ & 12.89960900 & 1.11785900 & 0.83128700 \\
\hline $\mathrm{N}$ & 8.46238100 & -0.23022200 & 0.53963700 \\
\hline $\mathrm{N}$ & -7.88205400 & -1.19032800 & 1.24796300 \\
\hline $\mathrm{N}$ & 0.11779600 & 1.81976200 & 0.28587400 \\
\hline
\end{tabular}

Number of imaginary frequency: 0 


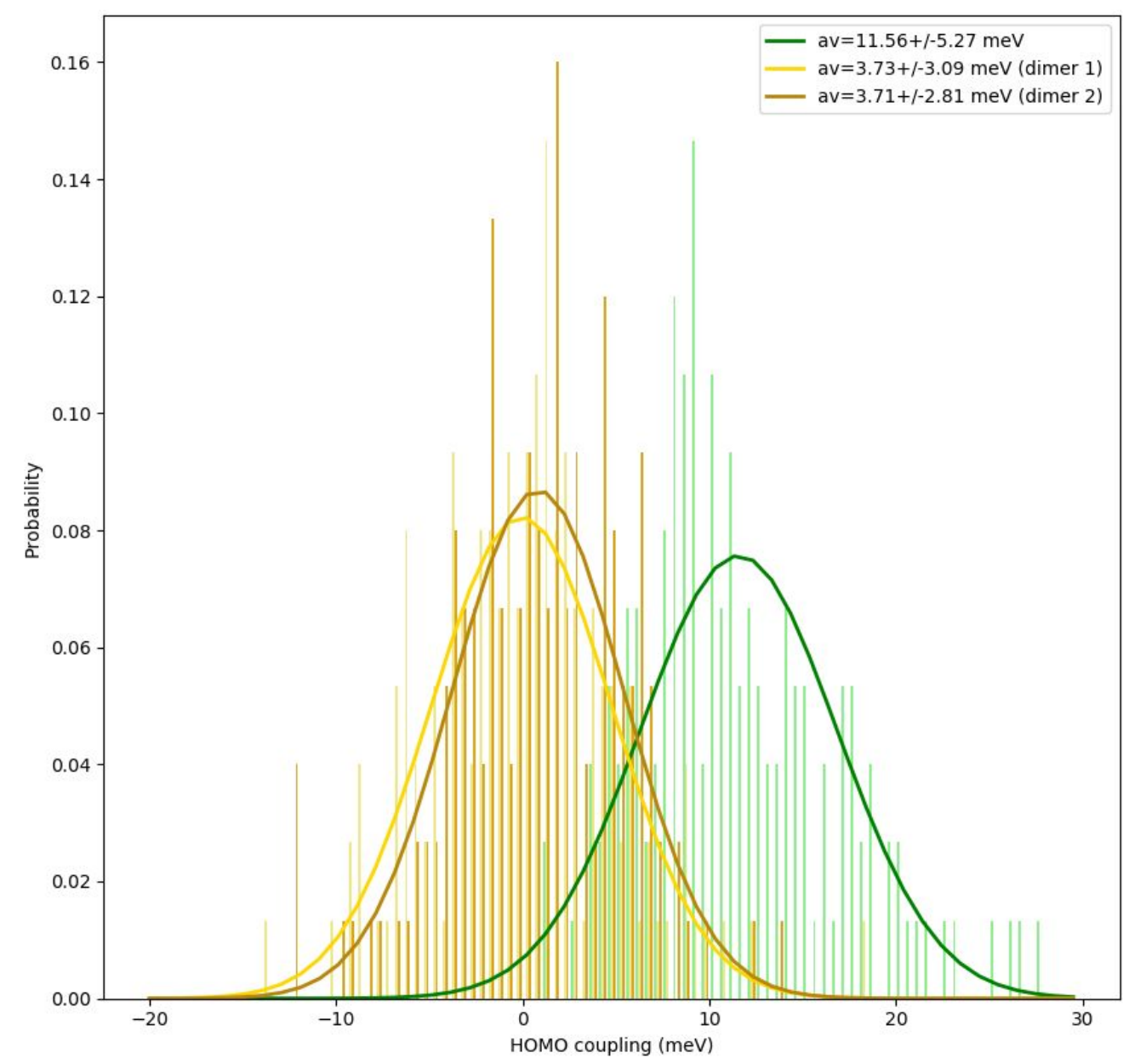

Figure S 33 Electronic coupling distribution and their respective Gaussian fits in [8/CPP. The green distribution corresponds to the green axis in Fig 9b. The two distributions in yellow and red are almost superposed, meaning that thermal noise affects one charge transfer direction as much as the other in the (bc) plane, see orange arrows in Fig.9. 


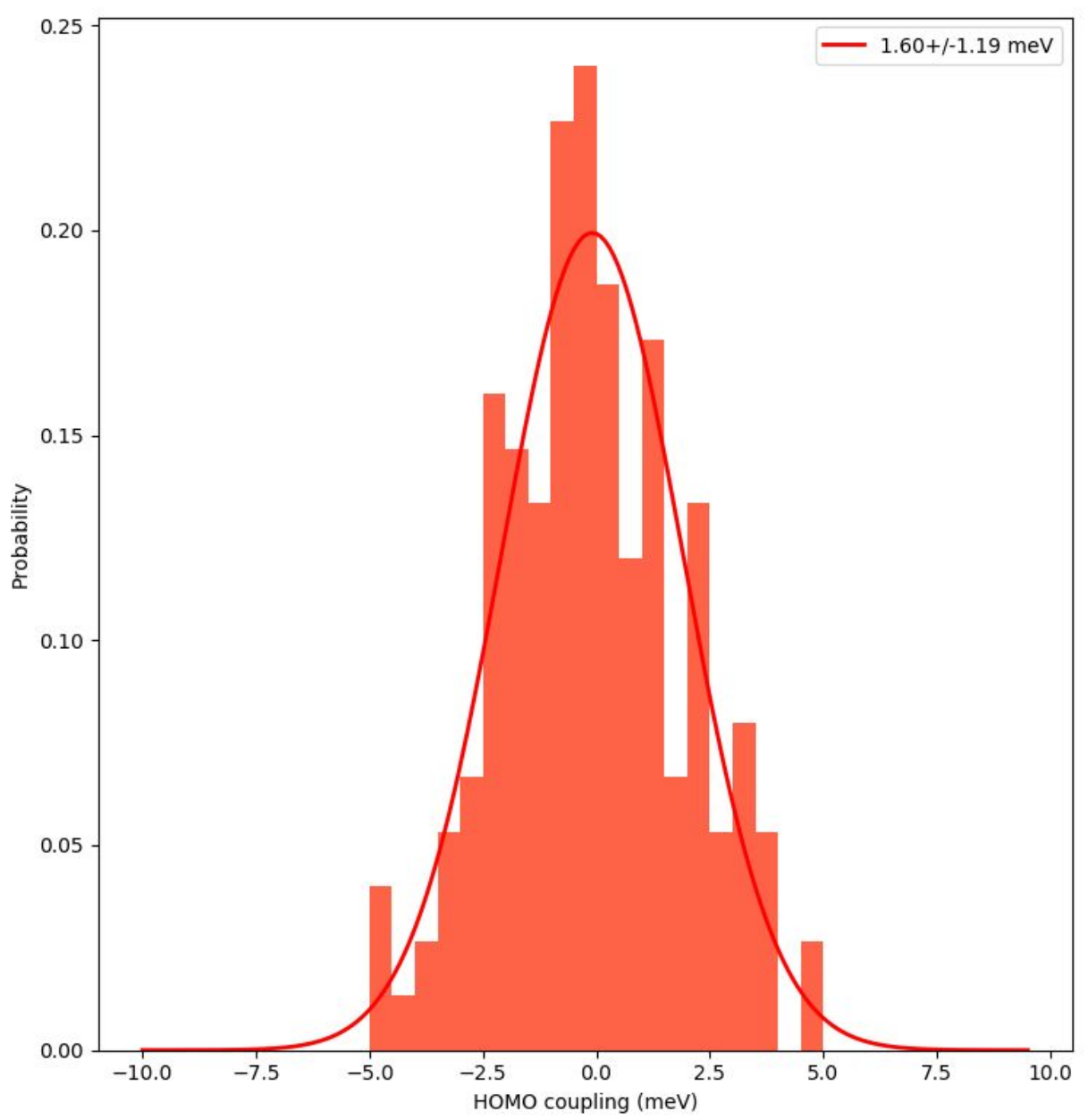

Figure $S 34$ Electronic coupling distribution in the zigzag molecular columns displayed of [4]C-Bu-Cbz displayed in Fig. 9. 


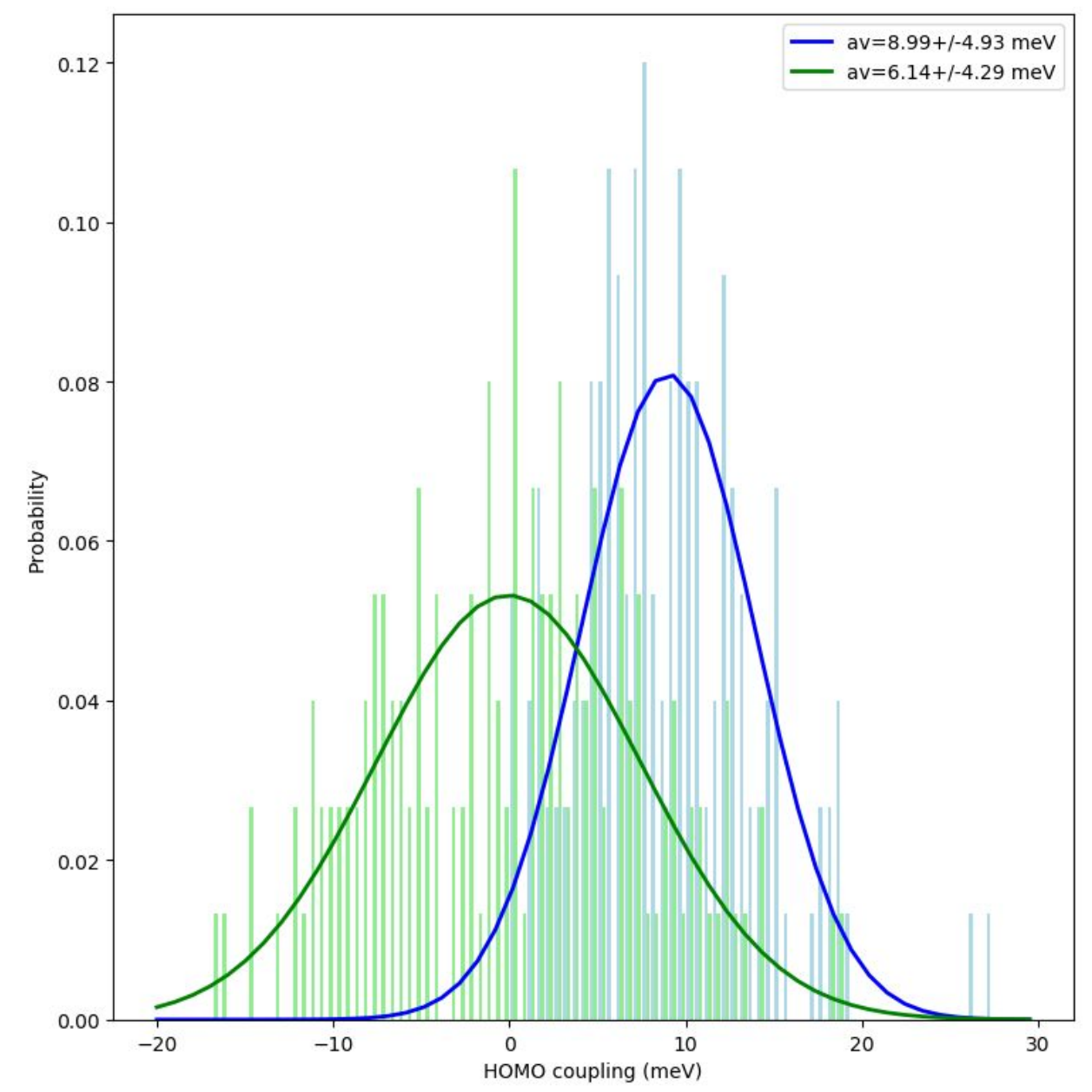

Figure $S 35$ Electronic coupling distributions in [4]C-Et-Cbz. The colors of the distribution are matched with the molecular columns in Fig. 9. Thermal noise affects both distribution differently. 
Table S 22 Summary of the important parameters implicated in the theoretical analysis

\begin{tabular}{|c|c|c|c|}
\hline Material & $\begin{array}{c}\text { X-ray structure } \\
\text { Value (meV) }\end{array}$ & $\begin{array}{c}\text { Molecular dynamics } \\
\text { Average value (meV) }\end{array}$ & $\begin{array}{c}\text { Molecular Dynamics } \\
\text { Standard deviation (meV) }\end{array}$ \\
\hline $\begin{array}{c}\text { [8]CPP green } \\
\text { molecular axis }\end{array}$ & 18.3 & 11.6 & 5.27 \\
\hline $\begin{array}{c}\text { [8]CPP yellow } \\
\text { plane (dimer 1) }\end{array}$ & 10.1 & 3.71 & 3.01 \\
\hline $\begin{array}{c}\text { [8]CPP yellow } \\
\text { plane (dimer 2) }\end{array}$ & 10.3 & 3.73 & 4.29 \\
\hline $\begin{array}{c}\text { [4]C-Et-Cbz green } \\
\text { axis }\end{array}$ & 10.8 & 6.14 & 4.93 \\
\hline $\begin{array}{c}\text { [4]C-Et-Cbz blue } \\
\text { axis }\end{array}$ & 11.6 & 8.99 & 1.19 \\
\hline [4]C-Bu-Cbz & 7.00 & 1.06 & \\
\hline
\end{tabular}




\section{X-ray diffraction structures and tables}

\section{[4]C-Et-Cbz}

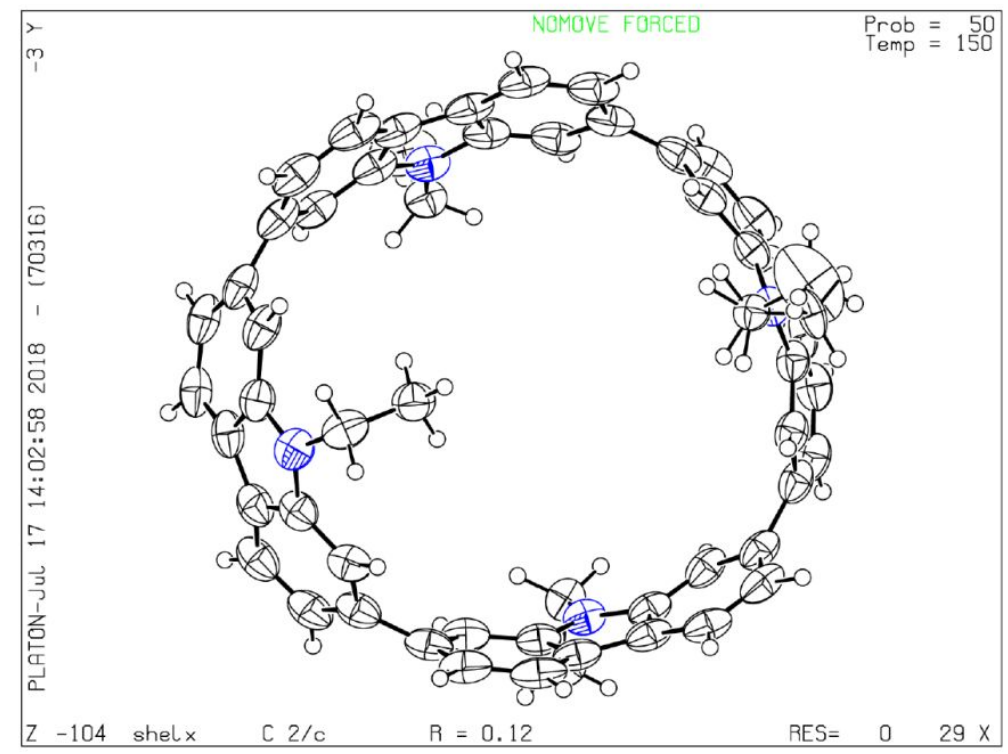

Table S 23 Crystal data and structure refinement for [4]C-Et-Cbz

\begin{tabular}{|c|c|}
\hline Identification code & shelx \\
\hline Empirical formula & $\mathrm{C} 57.25 \mathrm{H} 45.25 \mathrm{Cl} 3.75 \mathrm{~N} 4$ \\
\hline Formula weight & 922.16 \\
\hline Temperature & $150(2) \mathrm{K}$ \\
\hline Wavelength & $0.71073 \AA$ \\
\hline Crystal system & Monoclinic \\
\hline Space group & $\mathrm{C} 2 / \mathrm{c}$ \\
\hline \multirow{3}{*}{ Unit cell dimensions } & $\mathrm{a}=26.355(4) \AA \alpha=90^{\circ}$ \\
\hline & $\mathrm{b}=9.1895(13) \AA \beta=96.498(5)^{\circ}$ \\
\hline & $\mathrm{c}=41.286(6) \AA \gamma=90^{\circ}$ \\
\hline Volume & $9935(3) \AA 3$ \\
\hline $\mathrm{Z}$ & 8 \\
\hline Density (calculated) & $1.233 \mathrm{Mg} / \mathrm{m} 3$ \\
\hline Absorption coefficient & $0.266 \mathrm{~mm}-1$ \\
\hline $\mathrm{F}(000)$ & 3844 \\
\hline Crystal size & $0.13 \times 0.03 \times 0.02 \mathrm{~mm} 3$ \\
\hline Theta range for data collection & 2.349 to $27.637^{\circ}$ \\
\hline Index ranges & $\begin{array}{l}-32<=\mathrm{h}<=33, \quad-11<=\mathrm{k}<=10, \\
53<=\mathrm{k}<=53\end{array}$ \\
\hline Reflections collected & 46522 \\
\hline Independent reflections & $11248[\mathrm{R}(\mathrm{int})=0.0881]$ \\
\hline Completeness to theta $=25.242^{\circ}$ & $99.8 \%$ \\
\hline Refinement method & Full-matrix least-squares on F2 \\
\hline Data / restraints / parameters & $11248 / 3 / 653$ \\
\hline Goodness-of-fit on F2 & 1.093 \\
\hline Final $\mathrm{R}$ indices [I $>2 \operatorname{sigma}(\mathrm{I})]$ & $\mathrm{R} 1=0.1315, \mathrm{wR} 2=0.3529$ \\
\hline $\mathrm{R}$ indices (all data) & $\mathrm{R} 1=0.2068, \mathrm{wR} 2=0.4023$ \\
\hline Extinction coefficient & $0.0117(10)$ \\
\hline Largest diff. peak and hole & 2.164 and -0.604 e. $\AA$ - 3 \\
\hline
\end{tabular}




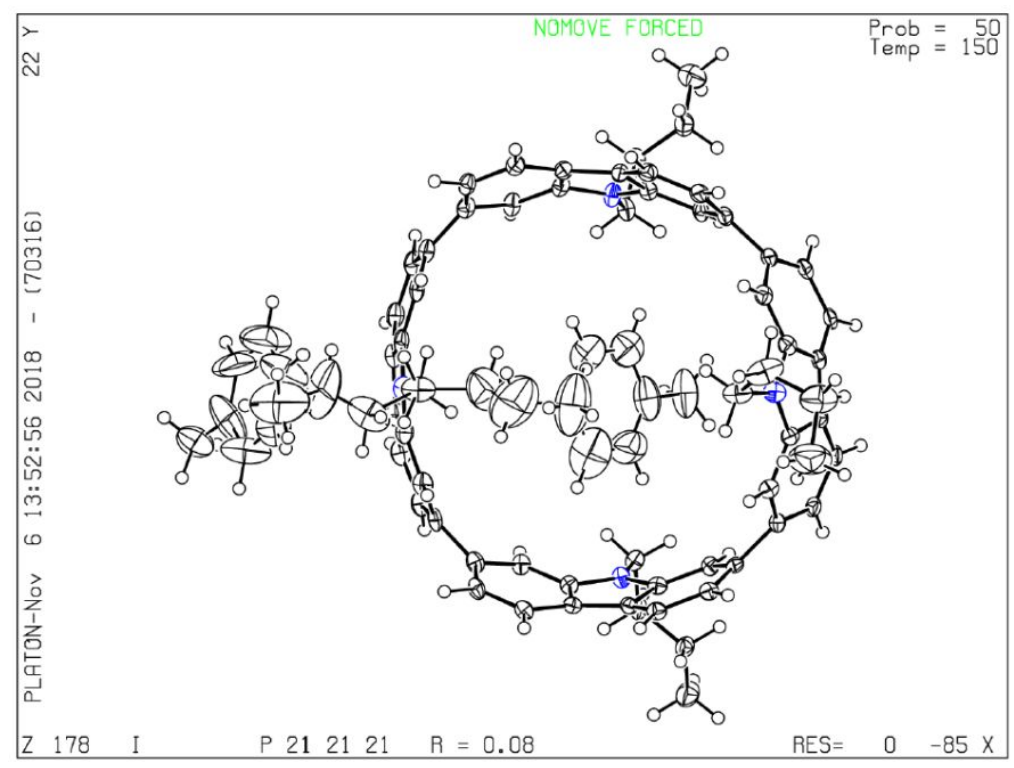

Table S 24 Crystal data and structure refinement for [4]C-Bu-Cbz

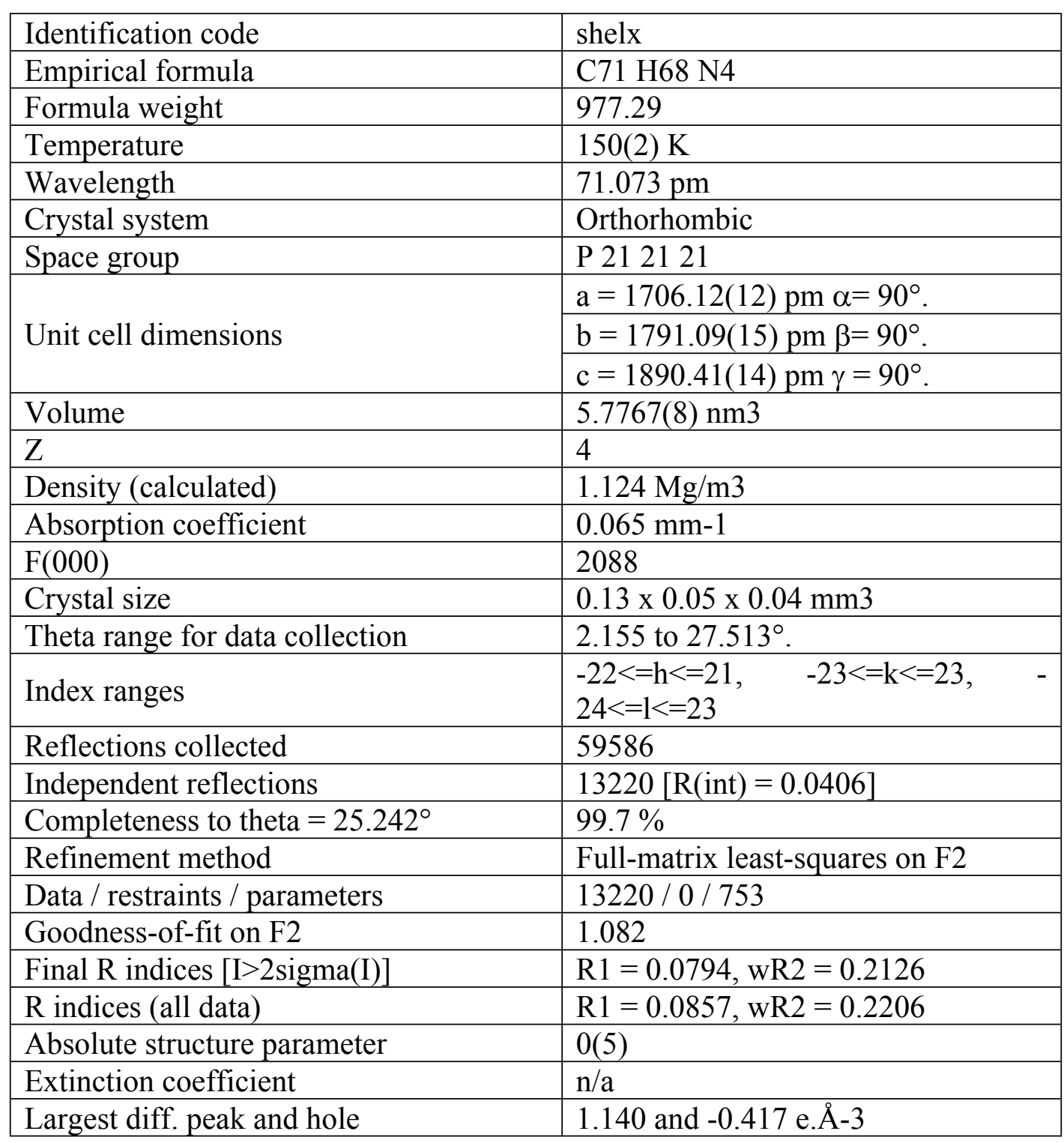




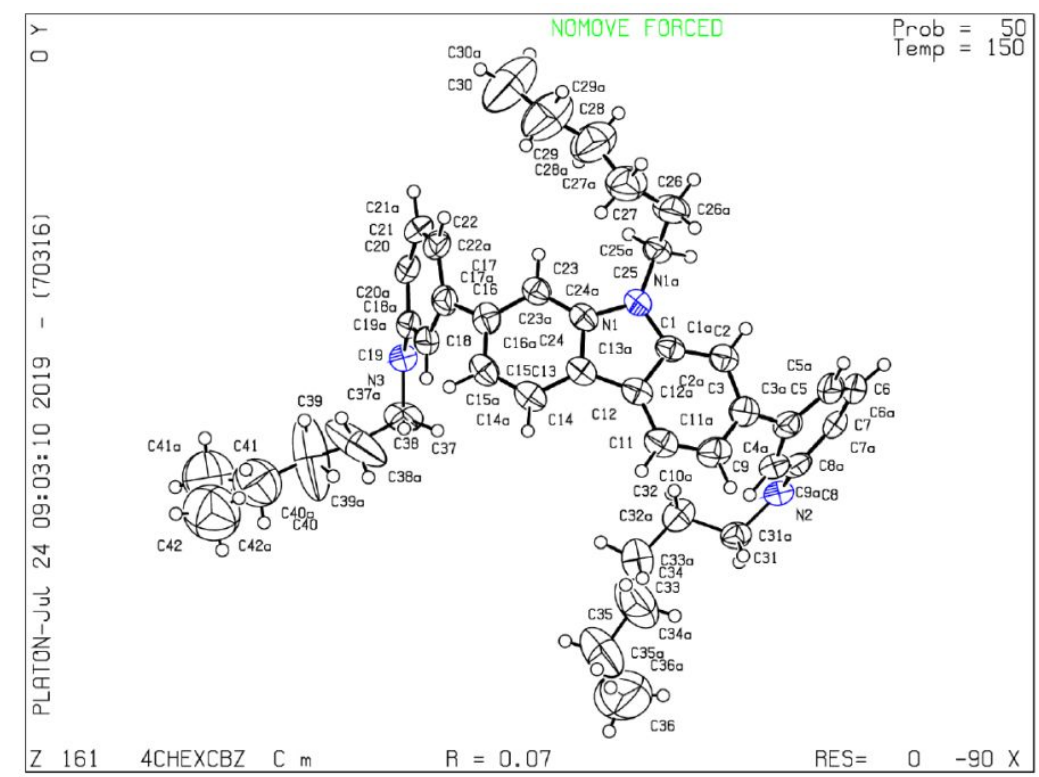

Table S 25 Crystal data and structure refinement for [4]C-Hex-Cbz

\begin{tabular}{|c|c|}
\hline Identification code & shelx \\
\hline Empirical formula & C72 H76 N4 \\
\hline Formula weight & 997.36 \\
\hline Temperature & $150(2) \mathrm{K}$ \\
\hline Wavelength & $0.71073 \AA$ \\
\hline Crystal system & Monoclinic \\
\hline Space group & $\mathrm{C} \mathrm{m}$ \\
\hline \multirow{3}{*}{ Unit cell dimensions } & $\mathrm{a}=9.4194(14) \AA \alpha=90^{\circ}$ \\
\hline & $\mathrm{b}=26.193(3) \AA \beta=101.590(5)^{\circ}$. \\
\hline & $\mathrm{c}=12.3056(17) \AA \gamma=90^{\circ}$ \\
\hline Volume & $2974.2(7) \AA 3$ \\
\hline $\mathrm{Z}$ & 2 \\
\hline Density (calculated) & $1.114 \mathrm{Mg} / \mathrm{m} 3$ \\
\hline Absorption coefficient & $0.064 \mathrm{~mm}-1$ \\
\hline $\mathrm{F}(000)$ & 1072 \\
\hline Crystal size & $0.160 \times 0.095 \times 0.070 \mathrm{~mm} 3$ \\
\hline Theta range for data collection & 2.296 to $27.796^{\circ}$ \\
\hline Index ranges & $\begin{array}{l}-12<=\mathrm{h}<=12, \quad-27<=\mathrm{k}<=34, \quad- \\
16<=\mathrm{l}<=15\end{array}$ \\
\hline Reflections collected & 21881 \\
\hline Independent reflections & $6886[\mathrm{R}(\mathrm{int})=0.0681]$ \\
\hline Completeness to theta $=25.242^{\circ}$ & $99.8 \%$ \\
\hline Refinement method & Full-matrix least-squares on F2 \\
\hline Data / restraints / parameters & $6886 / 60 / 401$ \\
\hline Goodness-of-fit on F2 & 1.047 \\
\hline Final $\mathrm{R}$ indices [I $>2 \operatorname{sigma}(\mathrm{I})]$ & $\mathrm{R} 1=0.0734, \mathrm{wR} 2=0.1879$ \\
\hline $\mathrm{R}$ indices (all data) & $\mathrm{R} 1=0.1116, \mathrm{wR} 2=0.2128$ \\
\hline Absolute structure parameter & $0(7)$ \\
\hline Extinction coefficient & $\mathrm{n} / \mathrm{a}$ \\
\hline Largest diff. peak and hole & 0.342 and -0.266 e. $. \AA-3$ \\
\hline
\end{tabular}


1. Altomare, A.; Cascarano, G.; Giacovazzo, C.; Guagliardi, A.; Burla, M. C.; Polidori, G.; Camalli, M., SIR92 - a program for automatic solution of crystal structures by direct methods. J. Appl. Cryst. 1994, 27 (3), 435-435.

2. Sheldrick, G., Crystal structure refinement with SHELXL. Acta Cryst. C 2015, 71 (1), 3-8.

3. Farrugia, L., WinGX and ORTEP for Windows: an update. J. Appl. Cryst. 2012, 45 (4), 849-854.

4. Kulkarni, A. P.; Tonzola, C. J.; Babel, A.; Jenekhe, S. A., Electron Transport Materials for Organic Light-Emitting Diodes. Chem. Mater. 2004, 16 (23), 4556-4573.

5. Kohn, P. H. a. W., Inhomogeneous Electron Gas. Phys. Rev. 136, B864.

6. Calais, J.-L., Density-Functional Theory of Atoms and Molecules. . Int. J. Quantum Chem. 1993, 47, 101.

7. Becke, A. D., A new mixing of Hartree-Fock and local density-functional theories $J$. Phys. Chem. 1993, 98, 1372.

8. Becke, A. D., Density-functional thermochemistry. III. The role of exact exchange $J$. Phys. Chem. 1993, 98, 5648.

9. Becke, A. D., Density-functional exchange-energy approximation with correct asymptotic behavior. Phys. Rev. A 1988, 38, 3098.

10. Lee C., Y. W. a. R. G. P., Development of the Colle-Salvetti correlation-energy formula into a functional of the electron density. phys. rev. B 1988, 37, 785.

11. M. J. Frisch, G. W. T., H. B. Schlegel, G. E. Scuseria, M. A. Robb, J. R. Cheeseman, G. Scalmani, V. Barone, B. Mennucci, G. A. Petersson, H. Nakatsuji, M. Caricato, X. Li, H. P. Hratchian, A. F. Izmaylov, J. Bloino, G. Zheng, J. L. Sonnenberg, M. Hada, M. Ehara, K. Toyota, R. Fukuda, J. Hasegawa, M. Ishida, T. Nakajima, Y. Honda, O. Kitao, H. Nakai, T. Vreven, J. A. J. Montgomery, J. E. Peralta, F. Ogliaro, M. Bearpark, J. J. Heyd, E. Brothers, K. N. Kudin, V. N. Staroverov, T. Keith, R. Kobayashi, J. Normand, K. Raghavachari, A. Rendell, J. C. Burant, S. S. Iyengar, J. Tomasi, M. Cossi, N. Rega, J. M. Millam, M. Klene, J. E. Knox, J. B. Cross, V. Bakken, C. Adamo, J. Jaramillo, R. Gomperts, R. E. Stratmann, O. Yazyev, A. J. Austin, R. Cammi, C. Pomelli, J. W. Ochterski, R. L. Martin, K. Morokuma, V. G. Zakrzewski, G. A. Voth, P. Salvador, J. J. Dannenberg, S. Dapprich, A. D. Daniels, O. Farkas, J. B. Foresman, J. V. Ortiz, J. Cioslowski, D. J. Fox, 2010, Gaussian 09, Revision B.01, Gaussian, Inc., Wallingford CT, 2010.

12. Segawa, Y.; Omachi, H.; Itami, K., Theoretical Studies on the Structures and Strain Energies of Cycloparaphenylenes. Org. Lett. 2010, 12 (10), 2262-2265.

13. Senthilkumar, K.; Grozema, F. C.; Bickelhaupt, F. M.; Siebbeles, L. D. A., Charge transport in columnar stacked triphenylenes: Effects of conformational fluctuations on charge transfer integrals and site energies. J. Chem. Phys. 2003, 119 (18), 9809-9817.

14. Rappe, A. K.; Goddard, W. A., Charge equilibration for molecular dynamics simulations. J. Phys. Chem. 1991, 95 (8), 3358-3363.

15. Hoyas, S.; Lemaur, V.; Duez, Q.; Saintmont, F.; Halin, E.; De Winter, J.; Gerbaux, P.; Cornil, J., PEPDROID: Development of a Generic DREIDING-Based Force Field for the Assessment of Peptoid Secondary Structures. Advanced Theory and Simulations 2018, 1 (12), 1800089.

16. MicroChem SU-8 2000 Permanent Epoxy Negative Photoresist Processing guidelines for SU-8 2000.5, SU-8 2002, SO-8 2005, SU-8 2007, SU-8 2010 and SU-8 2015. http://www.microchem.com/pdf/SU-82000DataSheet2000 5thru2015Ver4.pdf. 